\title{
Central Calorimeter Support Cradle Jack Failure Analysis
}

\author{
D-Zero Engineering Note 3740.215-EN-84 \\ D.L. Rudland \\ $4 / 10 / 87$
}

Approved Pulwabs 


\section{Introduction}

The Central Calorimeter and its support cradle (Figure 1) are to De supported by either hydraulic or mechanical jacks if hydraulics are used. each support will use two nydraulically coupled jacks with two out of the four supports nydraulically coupled giving the effect of a three point support system if mechanical Jacks are used, all four points are used for support Figure 2 shows two examples of jack placement on a 3.5 inch support plate

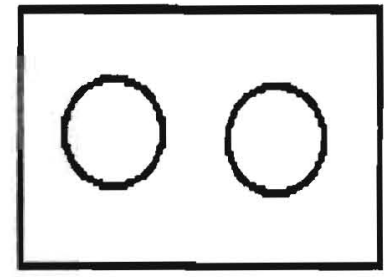

Hydraulically supported

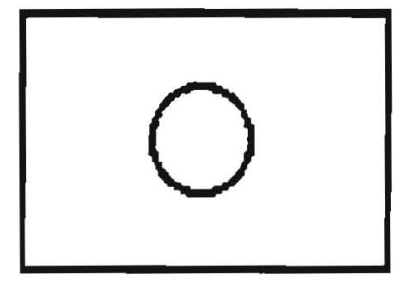

Mechanically supporter

Figure 2

These two support scenarios lead to five jack failure cases to be stlided. This report deals with the way in which a 0.25 inch drop (Failed jack) at one support affects the stresses in the cradle. The stresses from each failure case were analysed in two ways. First, stress factors, defined as quotients of stress intensities of the failed case with respect to the static case, were generated and then, hand calculations similar to those in Engineering Note 3740.215-EN-14 (Reference 1) were done using the reaction forces from the failed case

\section{The Five Failure Cases}

Viewing the Central Calorimeter Support Cradle from the top.

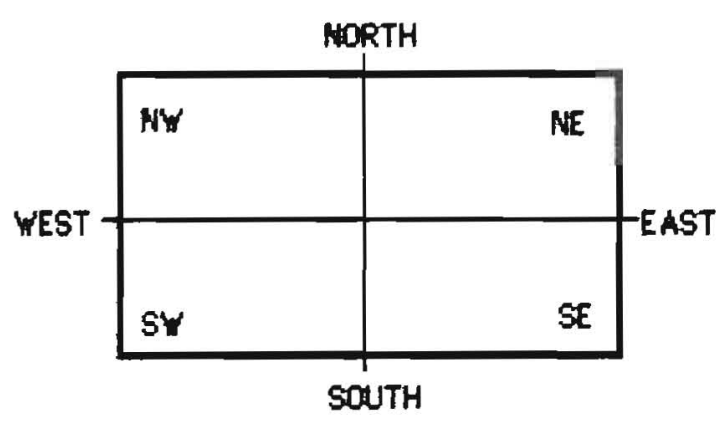


Where SE equals QUADRANT I, NE equals QUADRANT 2, SW equals QUADRANT 3, and NW equals QUADRANT 4. These are the four point of support. The five failure cases are as follows:

NUMBERONE: SW-SE Hydraulic Tie; NE Failure

NUMBERTWO: SW-SE Hydraulic Tie; SW-SE Failure

NUMBERTHREE: NW-SW Hydraulic Tie; NE Failure

NUMBERFOUR: NW-SW Hydraulic Tie; NW-SW Failure

NUMBERFIVE: ALL MECHANICAL; NE Failure

The failure cases and the quadrants noted above will be referred to by the above names for the remainder of this report.

\section{Finite Element Model}

Figure 3 shows a finite element model of the Central Calorimeter Support Cradle. For the hydraulic cases, the cradle is supported by tied beams (STIF4) and spars (STIF8) that ensure that hydraulically coupled supports will produce identical reaction forces, as do real hydraulics. The failed quadrant consists of a 'gap' element (STIF52) at each support node with a 0.25 inch initial gap. This element will allow a jack failure simulation at that quadrant. For the mechanical case, the cradle is supported by 0.00 inch initial gap spacers with 0.25 initial gap spacers at the failed quadrant. Loads and individual support arrangement are equivalent to that in Reference 1 and can be found in that note.

\section{Factor Listing}

Two FORTRAN programs (Appendix 1 and 2) were used to manipulate stress output from the finite element runs. The first, the Delete program, deletes all unnecessary headers from the stress printouts The second, called Program, takes the stress intensity of the static case and a falled case, divides them, and prints these factors and also the coordinates of each node. This list (approximately 1740 nodes) is reduced to the nodes that lie in the points of interest noted in Reference 1 (Section $A-A$, Section $B-B$, Tie Plate, Stanchion and 3.5 inch Support Plates, and the Arm connect) Figures 4-8 show the nodes chosen for this analysis. The program was also modified to pick out the factors that are greater than 12 and less than 0.75 . Appendices 3 through 7 contain the factor listings for cases NUMBERONE through NUMBERFIVE. 


\section{Cradle Jack Failure Analysis}

\section{Hand Calculations}

Stress calculations for the static case were done by Leo Engstrom in Reference 1. Stresses for each of the failure cases are tabulated in Table 2 of this report (Appendix 8 has the actual calculations). All of the calculations follow the exact format of Reference 1. The reactions used for these calculations are tabulated in Table 1 of this report and are ANSYS ${ }^{(0)}$ generated.

\section{Conclusion}

The hydraulic cases show that the reactions on the 3.5 inch support plate are well distributed thoughout the four plates. (Largest factor from table 1 is 1.047) Because of this, the hand calculations for the hydraulic cases are approximately equivalent to that of the static calculations in reference: The largest deviation from the static case $150.755 \mathrm{ks} 1$ in the bending stress of the $3.5 \mathrm{inch}$ bearing plate of case NUMBERONE. The largest factor for the hydraulic cases is 13302 and is found in the arm of the falled quadrant of case NUMBERONE. This factor corresponds to a change in stress from 539 psi to 717 psi.

The mechanical case however shows that the reactions do not distribute themselves as evenly ( Largest factor from table 1 is 1.17323 ). The calculations still do not vary greatly from that of the static case. The largest deviation from the static case is $2.82 \mathrm{ks}$ in the bearing stress of the 35 inch bearing plate of case NUMBERFIVE. The largest factor for the mechanical case is 4.1489 and appears in the arm of quadrant 1. This factor corresponds to a change in stress from 1214 psi to 5038 psi 


\section{References}
1.) 0-Zero Engineering Note $3740.215-E N-14$, Leo Engstrom. January 5,1987

\section{Appendices}

1.) Delete program *

2.) PROGRAM program *

3.) NUMBERONE Factor Listing *

4.) NUMBERT WO Factor Listing *

5.) NUMBERTHREE Factor Listing *

6.) NUMBEPFOUR Factor Listing *

7.) NUMBERFIVE Factor Listing *

8.) Hand Calculations

* Does not appear in distributed Engineering Note. See D-Zero Engineering Note Catalog for complete appendices 


\section{TABLE 1 REACTION COMPARISON}

\section{Reactions (Tons)}

NW NE $\quad$ SW $\quad$ SE

Static
Numberone
Numbertivo
Numberthree
Numberfour
Numberfive (hard)

89.113

93.3229

89.12

91.27

84.877

104.55
89.113

84.877

89.12

86.9547

93.323

73.65
89.113

89.1

89.12

91.27

84.877

73.65
89.113

89.1

89.12

86.944

93.323

104.55

(Bold is failed quadrent)

\section{Reaction Factors}
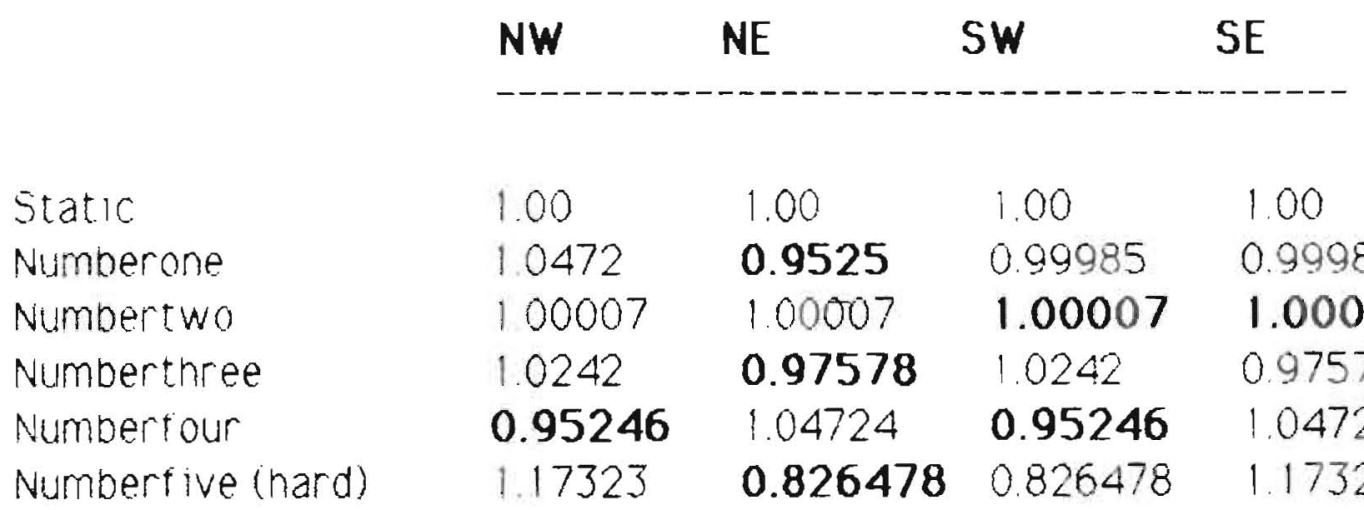

$\begin{array}{llll}1.00 & 1.00 & 1.00 & 1.00 \\ 1.0472 & 0.9525 & 0.99985 & 0.99985 \\ 1.00007 & 1.00007 & 1.00007 & 1.00007 \\ 1.0242 & 0.97578 & 1.0242 & 0.97578 \\ 0.95246 & 1.04724 & 0.95246 & 1.04724 \\ 1.17323 & 0.826478 & 0.826478 & 1.17323\end{array}$




\section{TABLE 2 CRADLE STRESSES (ksi)}

\begin{tabular}{|c|c|c|c|c|c|c|c|}
\hline Location & Allowable & Static & One & TwO & Three & Four & Five \\
\hline$-\cdots--$ & . & - & $\cdots$ & $\cdots$ & $\cdots \cdots$ & $---\infty$ & ------- \\
\hline \multicolumn{8}{|l|}{ Center Beam } \\
\hline Bending@B-B & 19.8 & 5.36 & 5.36 & 5.36 & 5.481 & 5.604 & 6.278 \\
\hline Bending@A-A & 19.8 & 7.52 & 7.867 & 7.52 & 7.694 & 7.867 & 8.813 \\
\hline Shear @B-B & 12.0 & 1.80 & 2.072 & 1.80 & 1.844 & 1.885 & 2.112 \\
\hline Shear @ A-A & 12.0 & 6.79 & 7.11 & 6.79 & 6.954 & 7.110 & 7.965 \\
\hline \multicolumn{8}{|l|}{ Arms } \\
\hline Beriding & 19.8 & 18.5 & 18.5 & 18.5 & 18.5 & 18.5 & 18.5 \\
\hline Shear & 12.0 & 9.91 & 9.91 & 9.91 & 9.91 & 9.91 & 9.91 \\
\hline \multicolumn{8}{|l|}{ Stanchion } \\
\hline Support Plate & 22.5 & 22.7 & 22.7 & 22.7 & 22.7 & 22.7 & 22.7 \\
\hline \multicolumn{8}{|l|}{3.5 in Bearing } \\
\hline \multicolumn{8}{|l|}{ Plate } \\
\hline Bending & 22.5 & 15.7 & 16.455 & 15.7 & 15.877 & 16.45 & 18.188 \\
\hline Plate Shear & 12.0 & 2.71 & 2.844 & 2.71 & 2.172 & 2.844 & 3.186 \\
\hline Punching Shear & 12.0 & 3.60 & 3.772 & 3.6 & 3.689 & 3.772 & 4.225 \\
\hline Bearing & 112.5 & 22.4 & 23.47 & 22.4 & 22.955 & 23.47 & 26.29 \\
\hline \multicolumn{8}{|l|}{ Tie Plate } \\
\hline Tension & 18.0 & 7.54 & 7.51 & 7.54 & 7.523 & 7.507 & 7.419 \\
\hline
\end{tabular}




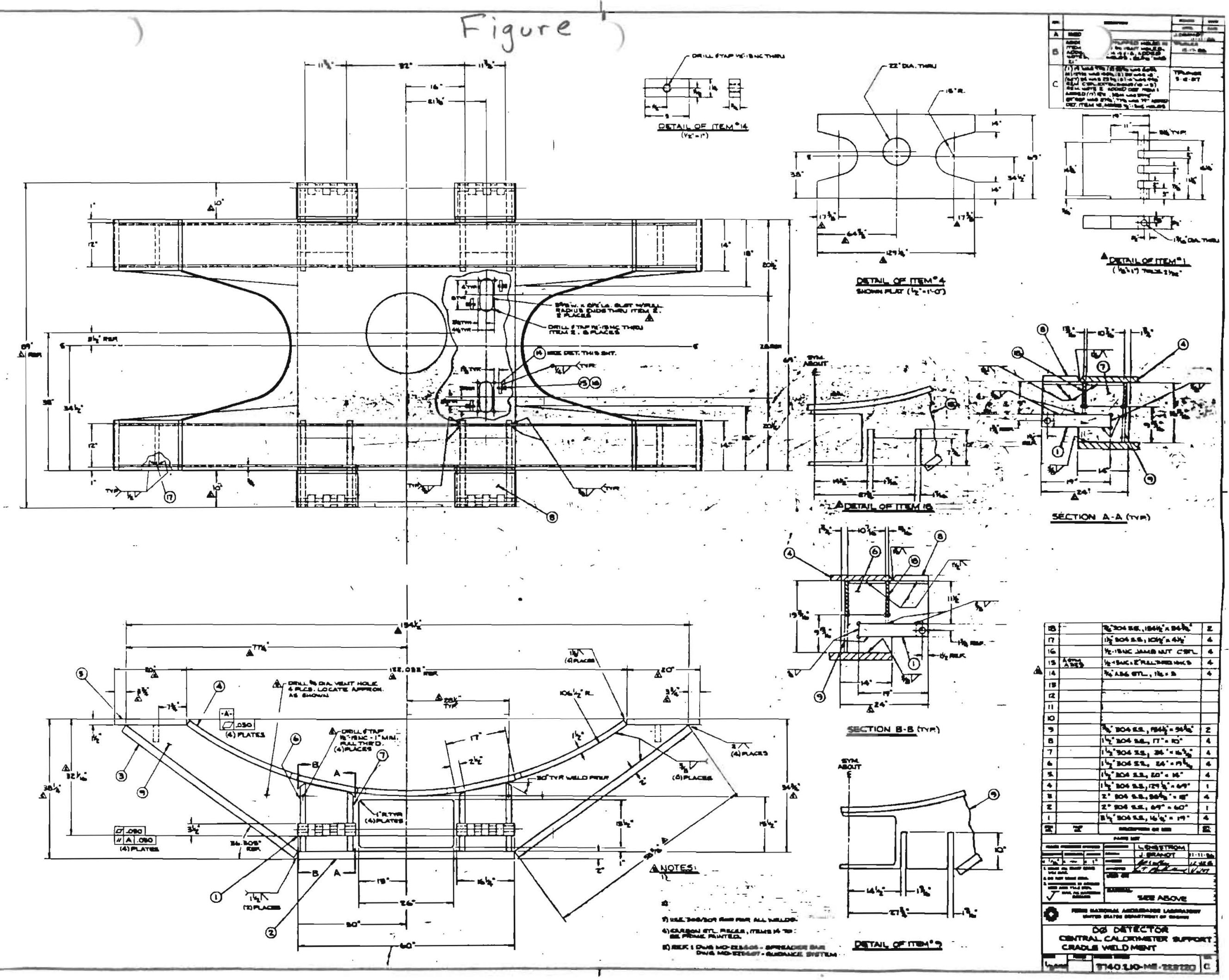




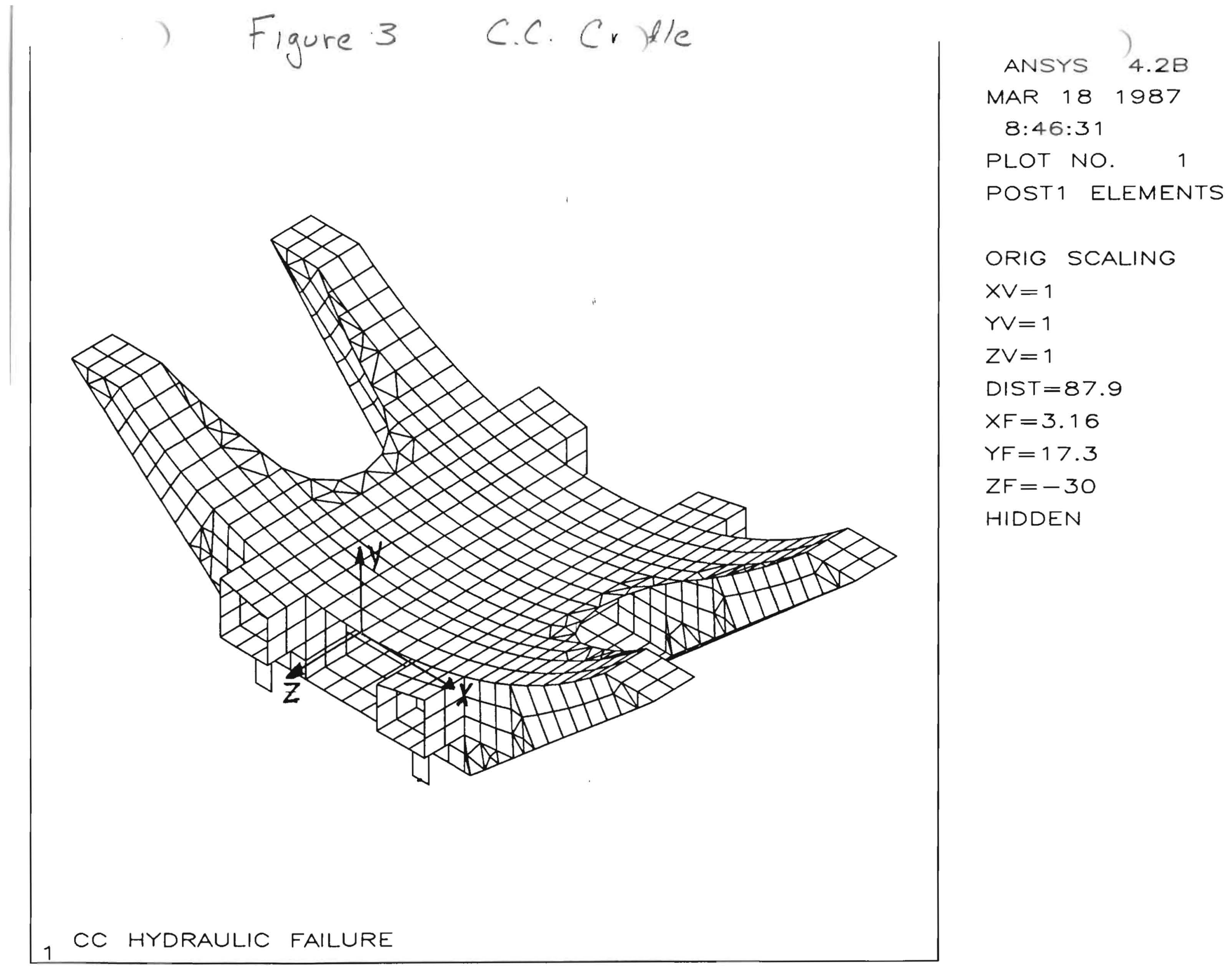




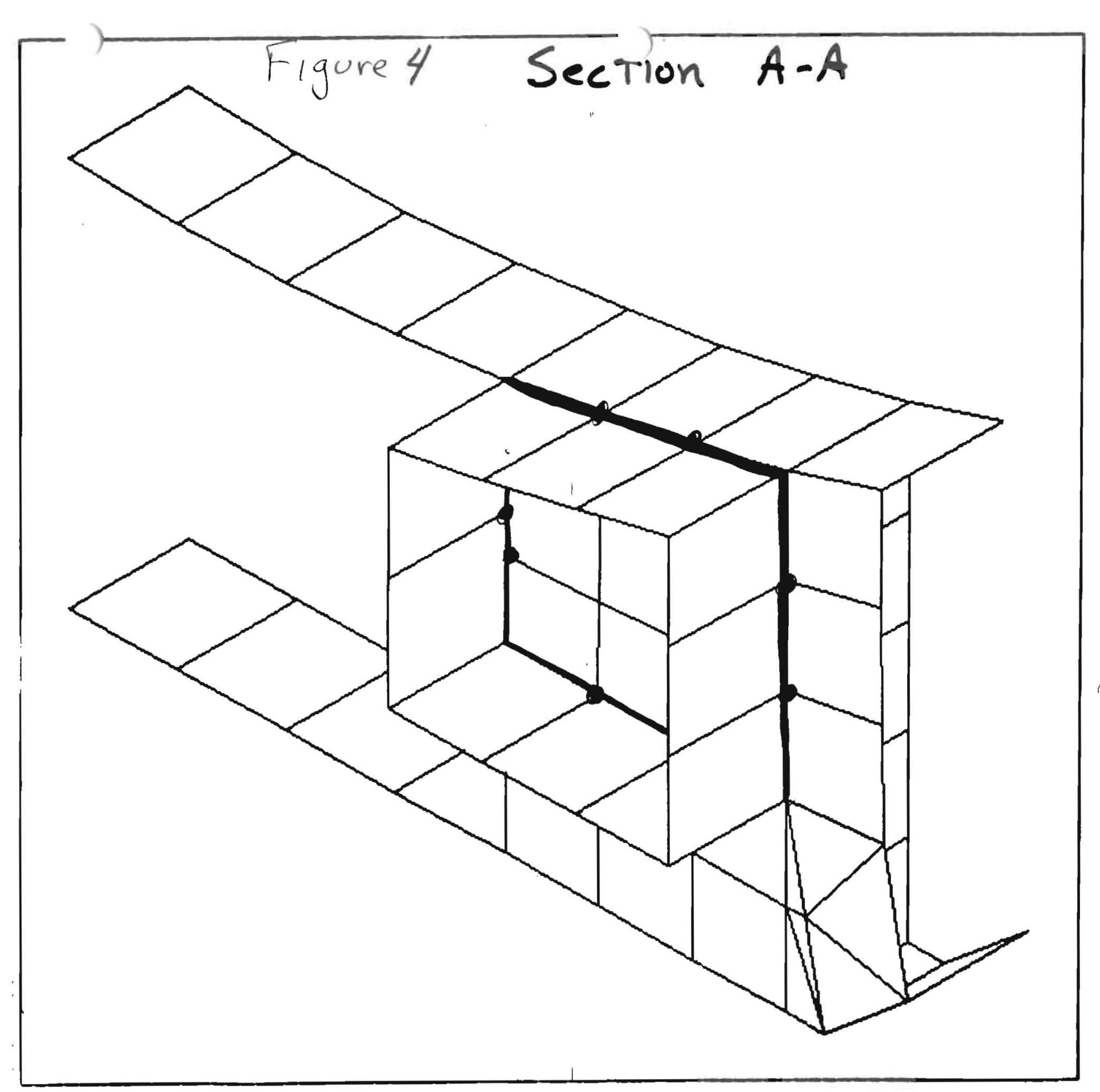




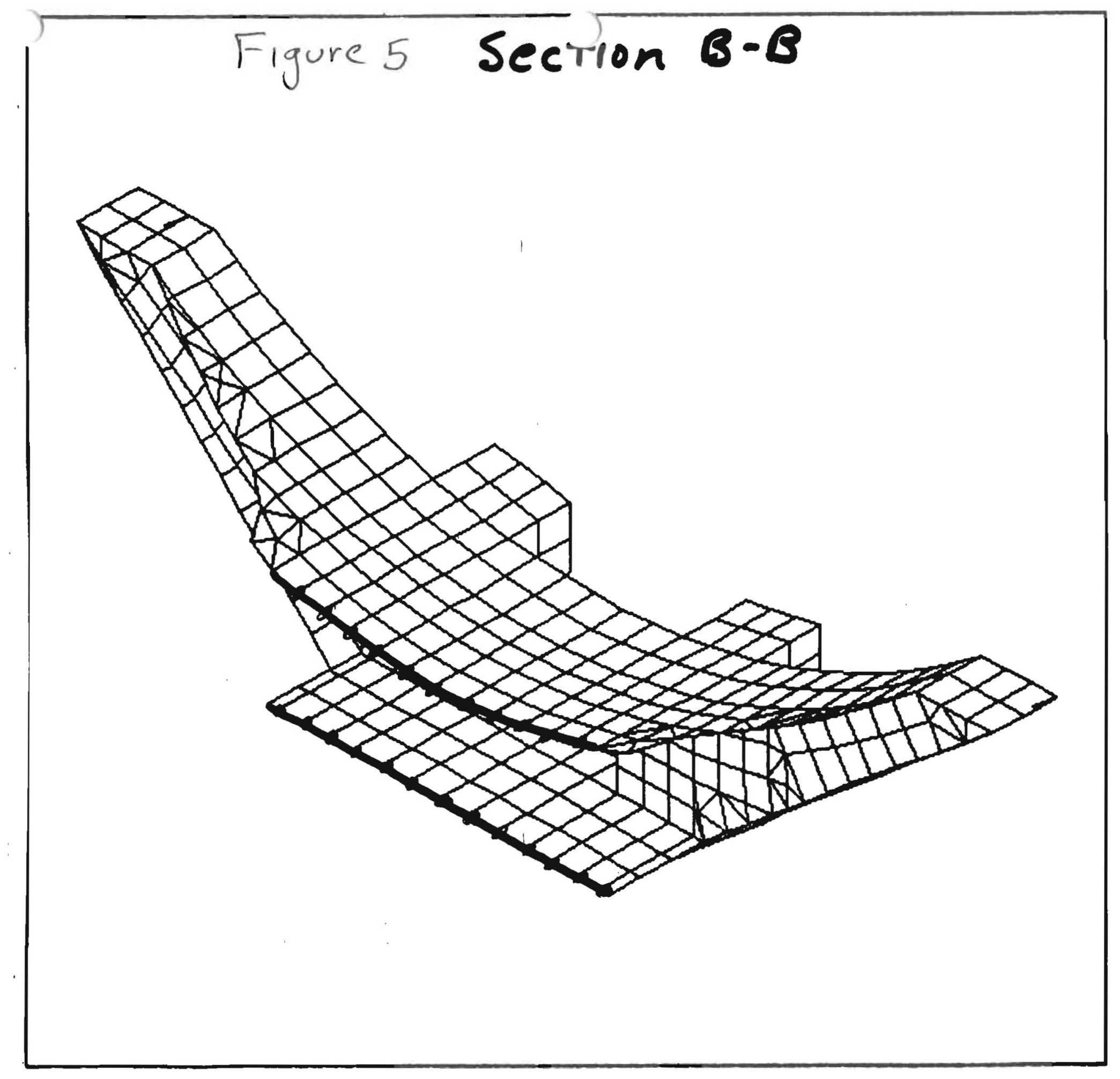




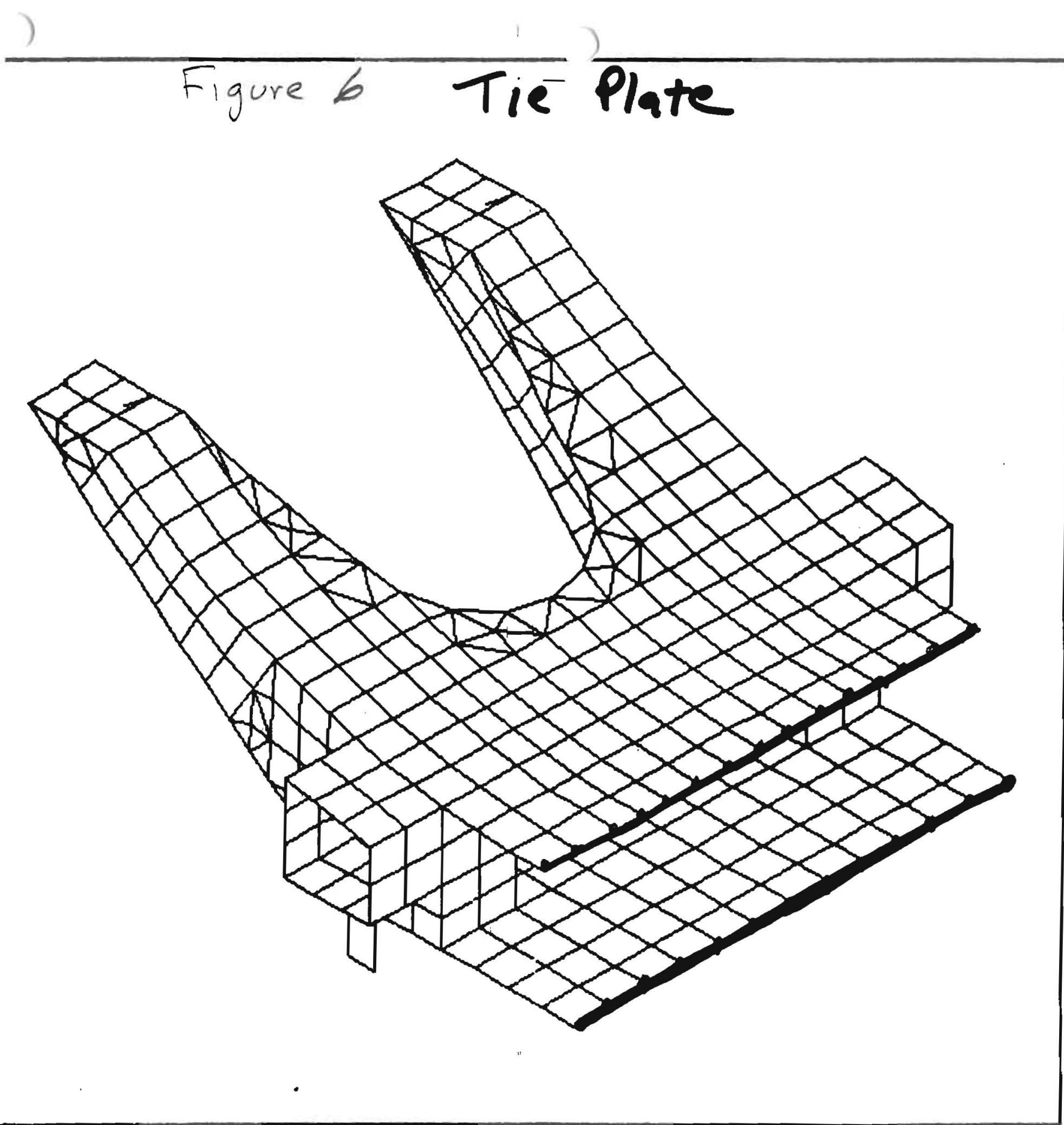


) Figure 7 stanchion: 3. Finch Support Plate
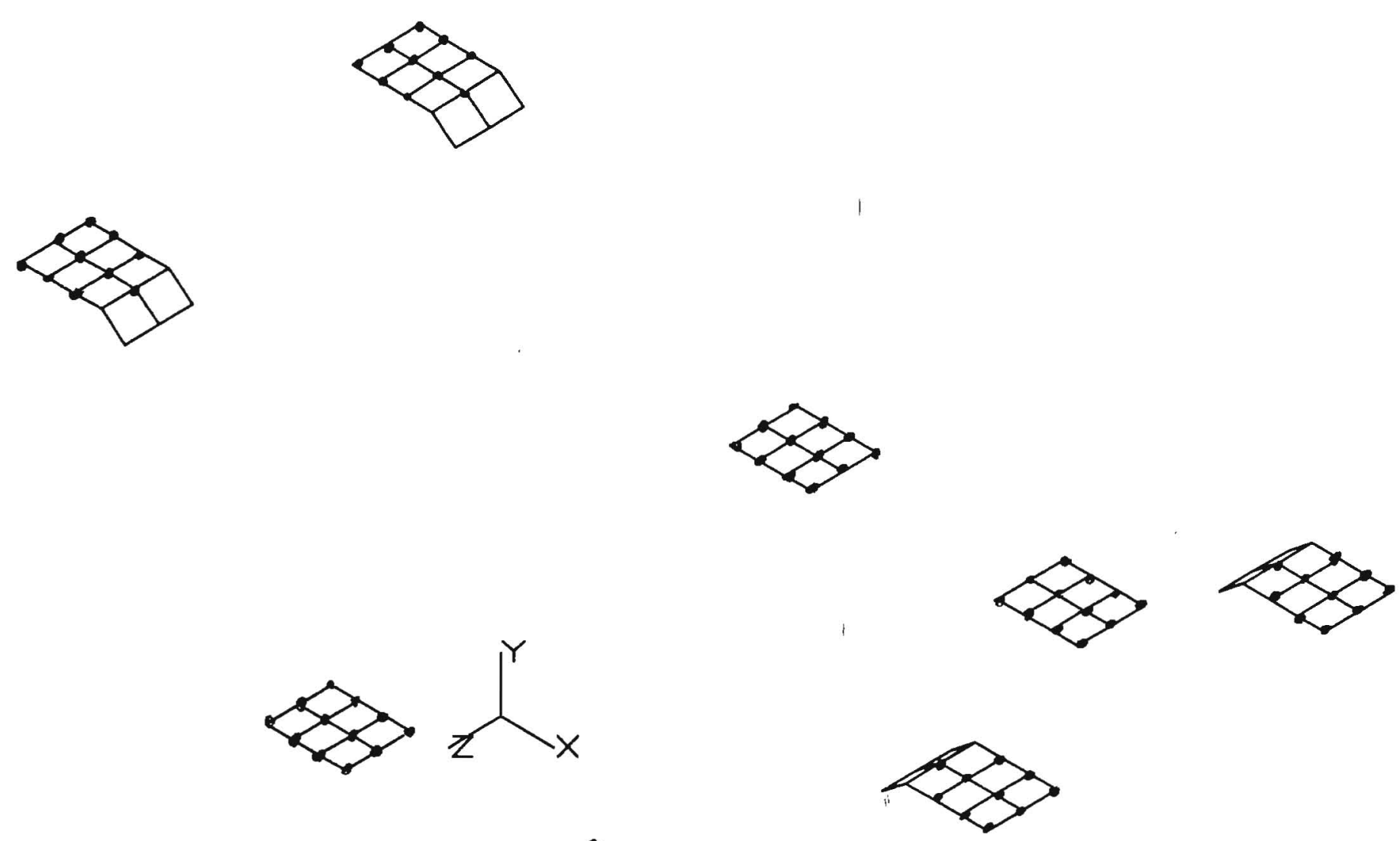

ANSYS

MAR $18 \quad 1987$

$8: 53: 15$

PLOT NO. 2

POST1 ELEMENTS

ORIG SCALING

$X V=1$

$Y V=1$

$Z V=1$

$\mathrm{DIST}=87.9$

$X F=2.14$

$Y F=19.3$

$Z F=-31$ 


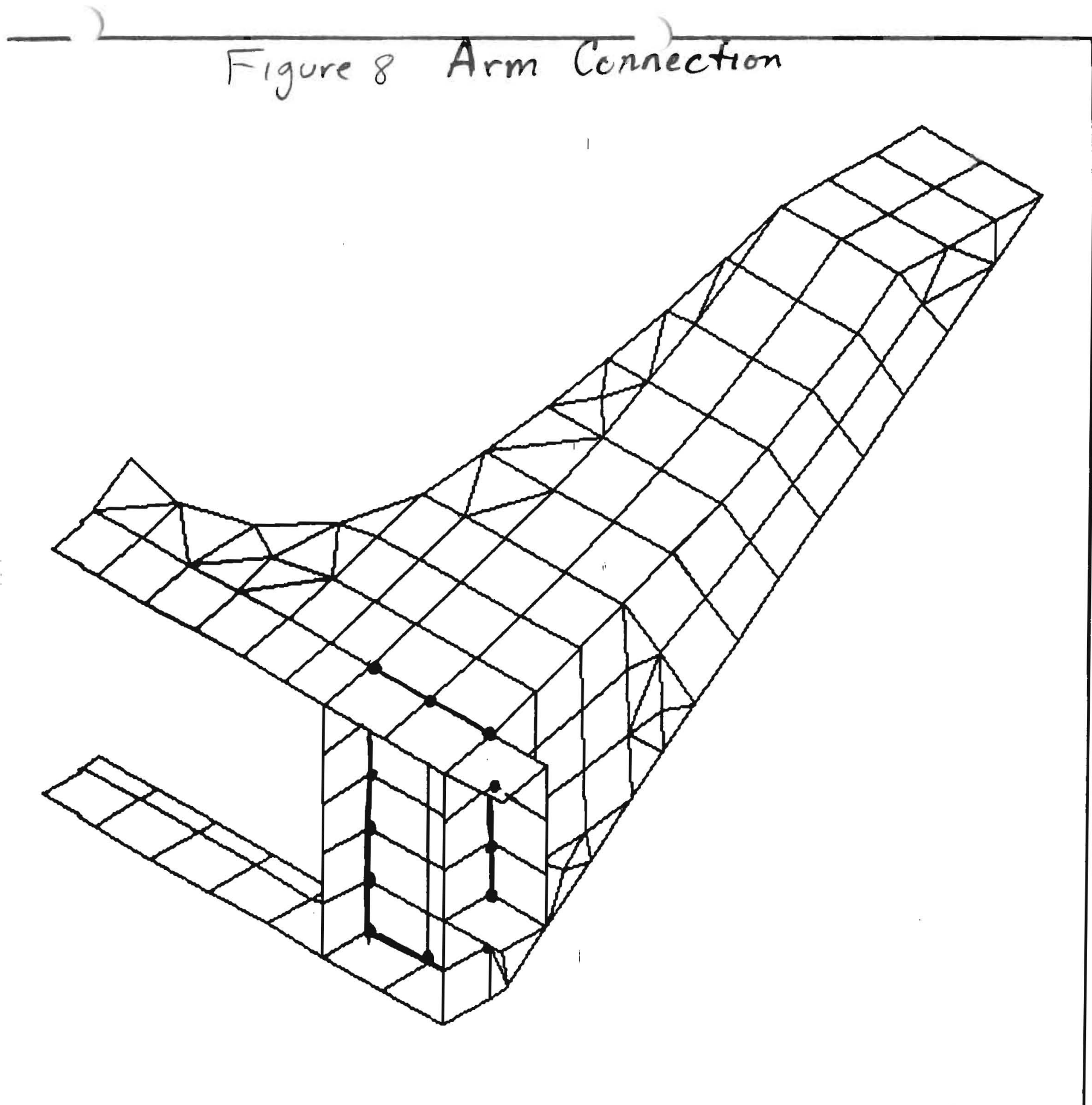


Appendix 1

DELETE Listing. 


\section{Delete}

c delete program

character $a * 80$

open (unit $=11$, status $={ }^{\prime}$ old', flle $={ }^{\prime}$ ONE2.DAT')

open(unit $=10$, status $={ }^{\prime}$ new', file $={ }^{\prime}$ OF INAL2.DAT')

read $(11,11)$ a

11 format(a80)

10 if a.eq.

) then

do $20 \quad i=1,7$

read $(11,11$, end $=90)$ a

20 continue

endif

write $(10,11)$ a

read $(11,11$, end $=90)$ a

goto 10

90 end 
APPENIDIX 2

PROGRAMListing. 


\section{PROGRAM}

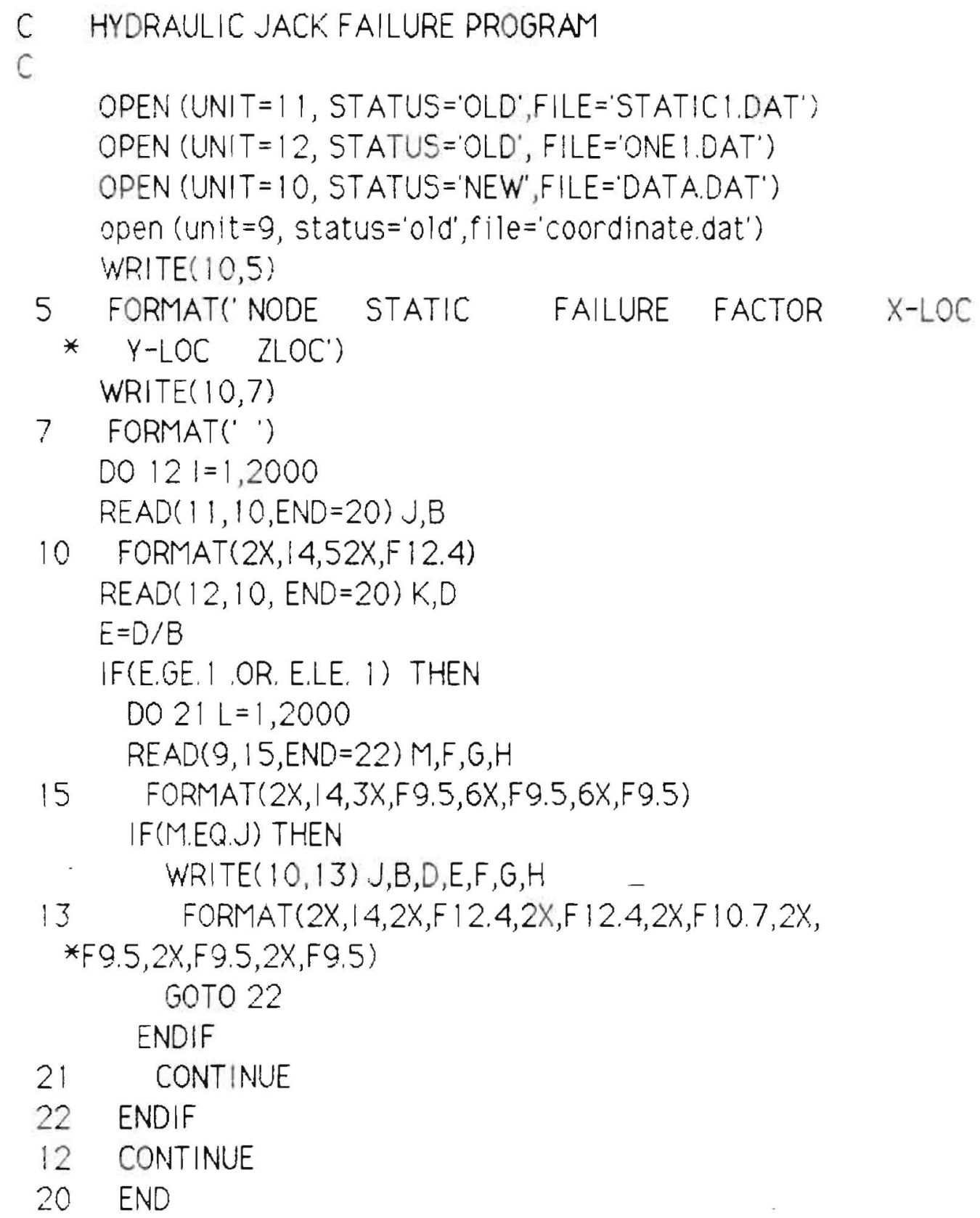




\section{APPENDDX 3 NUMBERONE}

FACTOR LISTING 


\section{NUMBERONE WORST}

$\begin{array}{lllllll}\text { NODE } & \text { STATIC } & \text { FAILURE } & \text { FACTOR } & \text { X-LOC } & \text { Y-LOC } & \text { Z-LOC } \\ & & & & & & \\ 511 & 5874.4043 & 4344.0947 & 0.7394953 & 15.25 & -9.645 & -66.25 \\ 513 & 6400.146 & 4795.0068 & 0.7492027 & 15.25 & -4.27 & -66.25 \\ 520 & 6356.3667 & 4602.0679 & 0.7240092 & 15.25 & -13.9475 & -60.625 \\ 534 & 539.0928 & 717.1074 & 1.3302114 & 19.5851 & 2.01868 & -77.031 \\ 539 & 5000.1997 & 3506.6101 & .7012940 & 15.25 & -9.645 & -71.64 \\ 541 & 5901.5444 & 3968.5972 & 0.6724675 & 15.25 & -4.27 & -71.64 \\ 545 & 4577.7803 & 5696.8579 & 1.2444586 & 28.2605 & -9.645 & -71.64 \\ 553 & 6224.6641 & 7869.0317 & 1.2641697 & 28.2605 & -5.14798 & -71.64 \\ 1589 & 2013.9762 & 2526.3550 & 1.2544116 & -15.2491 & -6.06167 & -66.25\end{array}$


SECTION A-A

\section{QUADRANT I}

NODE STATIC FAILURE FACTOR X-LOC Y-LOC Z-LOC

$\begin{array}{lllllll}14 & 11704.33 & 11553.21 & 0.9870883 & 19.6225 & 1.8365 & 0.000 \\ 15 & 9258.99 & 9171.51 & 0.9905517 & 23.9621 & 2.75057 & 0.000 \\ 113 & 6400.146 & 6527.3105 & 1.019869 & 15.2487 & -4.27 & 0.000 \\ 126 & 5711.4658 & 5540.5127 & 0.9700685 & 28.2605 & -5.14798 & 0.000 \\ 127 & 5161.3184 & 5069.1475 & 0.982142 & 28.2605 & -0.651 & 0.000 \\ 142 & 3779.1233 & 3622.7153 & 0.9586126 & 19.5868 & -9.645 & 0.000 \\ 143 & 6404.4614 & 6103.5308 & 0.9530123 & 23.9236 & -9.645 & 0.000 \\ 188 & 7970.8433 & 7623.667 & 0.956442 & 15.24827-2.47834 & 0.000 \\ 189 & 2013.9762 & 2153.5940 & 1.069324 & 15.24913 & -6.06167 & 0.000\end{array}$

QUADRANT 2

\begin{tabular}{|c|c|c|c|c|c|c|}
\hline$D E$ & STATIC & FAILURE & FACTOR & $X-L O C$ & $Y-L O C$ & LOC \\
\hline & & 1 & & & & \\
\hline & & & & & & \\
\hline & & 475 & & & -4.2 & \\
\hline & & 604 & & & -5 & \\
\hline & 84 & 544 & & & & \\
\hline & & & & & & \\
\hline & 4.461 & & & & -9 & \\
\hline & & & & & -2.47834 & \\
\hline & & 10 & & 91 & 6.06167 & \\
\hline
\end{tabular}

\section{QUADRANT 3}

$\begin{array}{lllllll}\text { NODE } & \text { STATIC } & \text { FAILURE } & \text { FACTOR } & \text { X-LOC } & \text { Y-LOC } & \text { Z-LOC } \\ 1014 & 11704.332011833 .8203 & 1.0110633 & -19.623 & 1.8365 & 0.000 \\ 1015 & 9258.99 & 9332.8838 & 1.007981 & -23.962 & 2.7506 & 0.000 \\ 1113 & 6400.146 & 6286.2134 & 0.9821984 & -15.248 & -4.27 & 0.000 \\ 1126 & 5711.4658 & 5871.5039 & 1.0280205 & -28.261 & -5.14798 & 0.000 \\ 1127 & 5161.3184 & 5244.3364 & 1.0160847 & -28.261 & -.06509 & 0.000 \\ 1142 & 3779.1233 & 3927.687 & 1.0393116 & -19.5868 & -9.645 & 0.000 \\ 1143 & 6404.4614 & 6684.3384 & 1.0437003 & -23.9236 & -9.645 & 0.000 \\ 1188 & 7970.8433 & 8287.6963 & 1.0397515 & -15.2483 & -2.47834 & 0.000 \\ 1189 & 2013.972 & 1893.4413 & 0.9401508 & -15.2491 & -6.06167 & 0.000\end{array}$




$\begin{array}{lllllll}\text { NODE } & \text { STATIC } & \text { FAILURE } & \text { FACTOR } & \text { X-LOC } & \text { Y-LOC } & \text { Z-LOC } \\ & & & & & & \\ 1414 & 11704.332 & 11199.2344 & 0.9568452 & -19.62251 .83647 & -66.25 \\ 1415 & 9258.9902 & 9140.8467 & 0.98724 & -23.9621 & 2.75057 & -66.25 \\ 1513 & 6400.146 & 6877.9766 & 1.0746593 & -15.25 & -4.27 & -66.25 \\ 1526 & 5711.4658 & 5594.793 & 0.9795722 & -28.261 & -5.147 & -66.25 \\ 1527 & 5161.3184 & 5121.1948 & 0.9922261 & -28.261 & -0.651 & -66.25 \\ 1542 & 3779.1233 & 3420.3391 & 0.9050615 & -19.587 & -9.645 & -66.25 \\ 1543 & 6404.461 & 5863.5654 & 0.915544 & -23.9236 & -9.645 & -66.25 \\ 1588 & 7970.8433 & 6781.145 & 0.850744 & -15.245 & -2.4783 & -66.25 \\ 1589 & 2013.97 & 2526.3550 & 1.254416 & -15.245 & -2.4783 & -66.25\end{array}$




$\begin{array}{lllllll}\text { NODE } & \text { STATIC } & \text { FAILURE } & \text { FACTOR } & \text { X-LOC } & \text { Y-LOC } & \text { Z-LOC } \\ & & & & & & \\ 75 & 1145.4335 & 1143.5752 & 0.998377 & 15.2474 & 1.1049 & -33125 \\ 78 & 1837.4819 & 1829.4562 & 0.9956322 & 0.41207 & 0.000 & -33.125 \\ 79 & 519.1904 & 555.8574 & 1.0706233 & 10.18418 & 0.49153 & -33.125 \\ 80 & 1419.4530 & 1423.5413 & 1.0028802 & 5.09876 & 0.12299 & -33.125 \\ 87 & 4898.3145 & 4819.0493 & 0.9833818 & 28.26045 & 3.84607 & -33.125 \\ 90 & 3179.0029 & 3122.3203 & 0.9821697 & 23.96206 & 2.75057 & -33.125 \\ 91 & 2120.7888 & 2088.0908 & 0.9845821 & 19.62247 & 1.83647 & -33.125 \\ 96 & 6835.5942 & 6741.04 & 0.9861674 & 31.97561 & 4.9501 & -33.125 \\ 216 & 937.4582 & 873.5552 & 0.931834 & 15.25 & -18.25 & -33.125 \\ 219 & 2182.5669 & 2169.4517 & 0.993991 & 0.000 & -18.28 & -33.125 \\ 220 & 1316.0106 & 1327.6467 & 1.008837 & 10.1667 & -18.25 & -33.125 \\ 221 & 1952.1735 & 1963.4034 & 1.0057525 & 5.08333 & -18.28 & -33.125 \\ 1075 & 1145.4335 & 1128.1584 & 0.9849184 & -15.248 & 1.10498 & -33.125 \\ 1079 & 519.1904 & 504.6033 & 0.9719041 & -10.1842 & 0.49153 & -33.125 \\ 1080 & 1419.4530 & 1514.3624 & 0.997118 & -5.0987 & .012299 & -33.125 \\ 1087 & 4898.3145 & 4988.8467 & 1.0184823 & -28.2604 & 3.84607 & -33.125 \\ 1090 & 3179.0029 & 3229.2168 & 1.015795 & -23.9621 & 2.75057 & -33.125 \\ 1091 & 2120.7888 & 2134.6372 & 1.0065298 & -19.622471 .83647 & -33.125 \\ 1096 & 6835.5942 & 6956.7769 & 1.0177282 & -31.9756 & 4.9501 & -33.125 \\ 1216 & 937.4582 & 973.9922 & 1.0389713 & -15.25 & -18.25 & -33.125 \\ 1220 & 1316.0605 & 1276.7382 & 0.9701211 & -10.1666 & -18.25 & -33.125 \\ 1221 & 1952.1735 & 1915.6520 & 0.9812919 & -5.08333 & -18.25 & -33.125 \\ 1242 & 4850.7827 & 4883.0703 & 1.0066562 & -23.9236 & -18.28 & -33.125 \\ 1243 & 2281.9146 & 2326.8164 & 1.0196773 & -19.58683 & -18.25 & -33.125\end{array}$




\section{tIE PLATE}

$\begin{array}{cllllll}\text { NODE } & \text { STATIC } & \text { FAILURE } & \text { FACTOR } & \text { X-LOC } & \text { Y-LOC } & \text { Z-LOC } \\ & & & & & & \\ 1 & 18590.941 & 18594.3438 & 1.000183 & 0.4121 & 0.000 & 0.000 \\ 7 & 13989.6982 & 13976.5576 & 0.9990607 & 0.4121 & 0.000 & -11.25 \\ 10 & 17322.6250 & 17312.5332 & 0.9994174 & 0.4121 & 0.000 & -5.625 \\ 51 & 6976.9146 & 6972.2427 & 0.9993304 & 0.4121 & 0.000 & -19.125 \\ 54 & 10640.6738 & 10630.5283 & 0.9990466 & 0.4121 & 0.000 & -15.187 \\ 78 & 1837.4819 & 1829.4562 & 0.9956322 & 0.4121 & 0.000 & -33.125 \\ 81 & 1768.3113 & 1760.3094 & 0.9954749 & 0.4121 & 0.000 & -28.791 \\ 82 & 3195.1631 & 3197.1777 & 1.0006305 & 0.4121 & 0.000 & -23.791 \\ 201 & 15208.0459 & 15216.2832 & 1.0005417 & 0.000 & -18.25 & 0.000 \\ 206 & 9185.1904 & 9187.2959 & 1.0002292 & 0.000 & -18.25 & -11.25 \\ 209 & 12347.4111 & 12350.4678 & 1.0002476 & 0.000 & -18.25 & -5.625 \\ 212 & 6175.9893 & 6176.7090 & 1.0001166 & 0.000 & -18.25 & -16.625 \\ 219 & 2182.5669 & 2169.4517 & 0.9939909 & 0.000 & -18.25 & -33.125 \\ 222 & 2146.0637 & 2132.5413 & 0.9936990 & 0.000 & -18.25 & -27.625 \\ 223 & 3621.4751 & 3617.8396 & 0.9989961 & 0.000 & -18.25 & -22.125 \\ 401 & 18590.9414 & 18233.9648 & 0.9807984 & 0.4121 & 0.000 & -66.25 \\ 407 & 13989.6982 & 13734.1768 & 0.9817351 & 0.4121 & 0.000 & -55.00 \\ 410 & 17322.625 & 17000.6660 & 0.981414 & 0.4121 & 0.000 & -60.625 \\ 451 & 6976.9146 & 6840.0576 & 0.980384 & 0.4121 & 0.000 & -47.125 \\ 454 & 10640.6738 & 10.439 .8135 & 0.9811233 & 0.4121 & 0.000 & -51.062 \\ 481 & 1768.3112 & 1769.7229 & 1.0008023 & 0.4121 & 0.000 & -37.791 \\ 482 & 3195.1631 & 3133.3098 & 0.9806416 & 0.4121 & 0.000 & -42.458 \\ 601 & 15208.0459 & 14918.5811 & 0.9809663 & 0.000 & -18.25 & -66.25 \\ 606 & 9185.1904 & 9016.8330 & 0.9816708 & 0.000 & -18.25 & -55.00 \\ 609 & 12347.4111 & 12120.8242 & 0.9816490 & 0.000 & -18.25 & -60.625 \\ 612 & 6175.9893 & 6058.0015 & 0.9808957 & 0.000 & -18.25 & -49.625 \\ 622 & 2146.0637 & 2123.7041 & 0.9895811 & 0.000 & -18.25 & -38.625 \\ 623 & 3621.4751 & 3545.2822 & 0.9789608 & 0.000 & -18.25 & -44.125\end{array}$




\title{
ARM CONNECT
}

\author{
QUADRANT' 1
}

$\begin{array}{lllllll}\text { NODE } & \text { STATIC } & \text { FAILURE } & \text { FACTOR } & \text { X-LOC } & \text { Y-LOC } & \text { Z-LOC } \\ 121 & 14662.2695 & 14972.5654 & 1.0211629 & 28.2605 & -9.645 & -11.25 \\ 124 & 9262.8857 & 9028.5322 & 0.9746997 & 28.2605 & -9.645 & 0.000 \\ 125 & 5323.7173 & 5250.1123 & 0.9861742 & 28.2605 & -9.645 & -5.625 \\ 181 & 10540.7070 & 10242.5664 & 0.9717153 & 28.2605 & -18.25 & 0.000 \\ 182 & 12551.1602 & 12196.5088 & 0.9717435 & 28.2605 & -13.6475 & 0.000 \\ 228 & 16030.9043 & 16094.2285 & 1.0039501 & 28.2605 & -18.25 & -11.25 \\ 231 & 8540.9580 & 8414.4404 & 0.9851869 & 28.2605 & -18.25 & -5.625 \\ 282 & 19003.9219 & 19318.3281 & 1.0165442 & 28.2605 & -13.9475 & -11.25 \\ 305 & 6782.7661 & 6634.0449 & 0.9780737 & 28.2605 & -13.9475 & -5.625\end{array}$

\section{QUADRANT 2}

$\begin{array}{lllllll}\text { NODE } & \text { STATIC } & \text { FAILURE } & \text { FACTOR } & \text { X-LOC } & \text { Y-LOC } & \text { Z-LOC } \\ & & & & & & \\ 521 & 14662.2695 & 14128.0537 & 0.9635653 & 28.2605 & -9.645 & -55.00 \\ 524 & 9262.8857 & 9906.2305 & 1.0694541 & 28.2605 & -9.645 & -66.25 \\ 525 & 5323.7173 & 5825.7344 & 1.0942982 & 28.2605 & -9.945 & -60.625 \\ 581 & 10540.7070 & 10491.7090 & 0.9953516 & 28.2605 & -18.25 & -66.25 \\ 582 & 12551.1602 & 12846.7617 & 1.0235517 & 28.26050 & -13.9475 & -66.25 \\ 628 & 16030.9043 & 15977.2119 & 0.9966507 & 28.26050 & -18.25 & -55.00 \\ 631 & 8540.9580 & 8811.1035 & 1.0316294 & 28.2605 & -18.25 & -60.625 \\ 682 & 19003.9219 & 18502.3125 & 0.973605 & 28.2605 & -13.9475 & -55.00 \\ 705 & 6782.7661 & 7508.6611 & 1.1070205 & 28.2605 & -13.9475 & -60.625\end{array}$

\section{QUADRANT 3}

$\begin{array}{lllllll}\text { NODE } & \text { STATIC } & \text { FAILURE } & \text { FACTOR } & \text { X-LOC } & \text { Y-LOC } & \text { Z-LOC } \\ 1121 & 16442.2695 & 14346.7080 & 0.9784780 & -28.2605 & -9.645 & -11.25 \\ 1124 & 9262.8857 & 9485.6621 & 1.0240505 & -28.2605 & -9.645 & 0.000 \\ 1125 & 5323.7173 & 5390.4409 & 1.0125333 & -28.2605 & -9.645 & -5.625 \\ 1181 & 10540.7070 & 10833.9590 & 1.0278209 & -28.2605 & -18.25 & 0.000 \\ 1182 & 12551.1602 & 12891.3643 & 1.0271053 & -28.2605 & -13.9475 & 0.000 \\ 1228 & 16030.9043 & 15938.1523 & 0.9942142 & -28.2605 & -18.25 & -11.25 \\ 1231 & 8540.9580 & 8658.4346 & 1.01375454 & -28.2605 & -18.25 & -5.625 \\ 1282 & 19003.9219 & 18671.4492 & 0.982505 & -28.2605 & -13.9475 & -11.25 \\ 1305 & 6782.7661 & 6922.4951 & 1.0206006 & -28.2605 & -13.9475 & -5.625\end{array}$




\section{QUADRANT 4}

$\begin{array}{lllllll}\text { NODE } & \text { STATIC } & \text { FAILURE } & \text { FACTOR } & \text { X-LOC } & \text { Y-LOC } & \text { Z-LOC } \\ & & & & & & \\ 1521 & 14662.2695 & 14678.2686 & 1.0010911 & -28.2605 & -9.6450 & -55.00 \\ 1524 & 9262.8857 & 9138.5430 & 0.9865763 & -28.2605 & -9.645 & -66.25 \\ 1525 & 5325.7173 & 5322.3130 & 0.9997362 & -28.2605 & -9.645 & -60.625 \\ 1581 & 10540.7070 & 10303.2646 & 0.9774738 & -28.2605 & -18.25 & -66.25 \\ 1582 & 12551.1602 & 12300.6240 & 0.9800388 & -28.2605 & -13.9475 & -66.25 \\ 1628 & 16030.9043 & 16093.0664 & 1.0038776 & -28.2605 & -18.25 & -55.00 \\ 1631 & 8540.9580 & 8451.1113 & 0.9894805 & -28.2605 & -18.25 & -60.625 \\ 1682 & 19003.9219 & 19135.8457 & 1.0069419 & -28.2605 & -13.9475 & -55.00 \\ 1705 & 6782.7661 & 6722.1953 & 0.9910699 & -28.2605 & -13.9475 & -60.625\end{array}$




\section{STANCHION AND 3.5 INCH SUPPORT PLATES}

\section{QUADRANT 1}

NODE STATIC FAILURE FACTOR X-LOC Y-LOC ZLOC

$\begin{array}{lllllll}38 & 14083.2598 & 14086.7832 & 1.0002502 & 66.62949 & 23.63070 & -5.62500\end{array}$

$\begin{array}{lllllll}100 & 4698.7598 & 4686.8618 & 0.9974679 & 79.90000 & 23.63070 & 0.00000\end{array}$

$\begin{array}{lllllll}101 & 8676.7129 & 8671.6094 & 0.9994118 & 71.05299 & 23.63070 & 0.00000\end{array}$

$\begin{array}{lllllll}102 & 7697.5327 & 7671.7847 & 0.9966550 & 75.47649 & 23.63070 & 0.00000\end{array}$

$103 \quad 6581.2065 \quad 6578.6240 \quad 0.9996076 \quad 79.90000 \quad 23.63070-11.25000$

$\begin{array}{lllllll}104 & 7036.5752 & 7045.7700 & 1.0013068 & 79.90000 & 23.63070 & -5.62500\end{array}$

$\begin{array}{lllllll}105 & 13235.8369 & 13264.4111 & 1.0021589 & 75.47649 & 23.63070 & -11.25000\end{array}$

$\begin{array}{lllllll}106 & 15801.0771 & 15854.1289 & 1.0033575 & 71.05299 & 23.63070 & -11.25000\end{array}$

$\begin{array}{lllllll}107 & 21384.1797 & 21390.2676 & 1.0002847 & 71.05299 & 23.63070 & -5.62500\end{array}$

$\begin{array}{lllllll}108 & 15424.8838 & 15429.3447 & 1.0002892 & 75.47649 & 23.63070 & -5.62500\end{array}$

$\begin{array}{lllllll}111 & 7406.6182 & 7465.0781 & 1.0078930 & 15.25000 & -9.64501 & 0.00000\end{array}$

$\begin{array}{lllllll}124 & 9620.8887 & =9365.8760 & 0.9734939 & 28.26050 & -9.64501 & 0.00000\end{array}$

$\begin{array}{lllllll}138 & 6601.4473 & 6950.9175 & 1.0529385 & 15.25000 & -9.64501 & 10.78100\end{array}$

$\begin{array}{lllllll}139 & 9114.5664 & 9287.4629 & 1.0189693 & 15.25000 & -9.64501 & 5.39050\end{array}$

$\begin{array}{lllllll}142 & 6885.4316 & 6741.9795 & 0.9791659 & 19.58683 & -9.64501 & 0.00000\end{array}$

$\begin{array}{lllllll}143 & 7686.2979 & 7411.7759 & 0.9642842 & 23.92366 & -9.64501 & 0.00000\end{array}$

$\begin{array}{lllllll}144 & 7939.5073 & 7634.2236 & 0.9615488 & 28.26050 & -9.64501 & 10.78100\end{array}$

$\begin{array}{lllllll}145 & 10971.8213 & 10759.5469 & 0.9806527 & 28.26050 & -9.64501 & 5.39050\end{array}$

$\begin{array}{lllllll}146 & 11031.4912 & 11038.5010 & 1.0006354 & 23.92366 & -9.64501 & 10.78100\end{array}$

$\begin{array}{llllllll}147 & 11312.3691 & 11269.5176 & 0.9962119 & 19.58683 & -9.64501 & 10.78100\end{array}$

$\begin{array}{lllllll}148 & 14395.0352 & 14401.6133 & 1.0004569 & 19.58683 & -9.64501 & 5.39050\end{array}$

$\begin{array}{llllllll}149 & 14264.2383 & 14241.3594 & 0.9983960 & 23.92366 & -9.64501 & 5.39050\end{array}$ 
QUADRANT 2

NODE STATIC FAILURE FACTOR X-LOC Y-LOC ZLOC

$\begin{array}{lllllll}438 & 14083.2598 & 14077.9971 & 0.9996263 & 66.62949 & 23.63070 & -60.62500\end{array}$

$\begin{array}{lllllll}500 & 4698.7598 & 4717.7686 & 1.0040455 & 79.90000 & 23.63070 & -66.25000\end{array}$

$\begin{array}{lllllll}501 & 8676.7129 & 8681.0176 & 1.0004961 & 71.05299 & 23.63070 & -66.25000\end{array}$

$\begin{array}{lllllll}502 & 7697.5327 & 7736.8345 & 1.0051057 & 75.47649 & 23.63070 & -66.25000\end{array}$

$\begin{array}{lllllll}503 & 6581.2065 & 6587.2222 & 1.0009141 & 79.90000 & 23.63070 & -55.00000\end{array}$

$\begin{array}{lllllll}504 & 7036.5752 & 7023.4785 & 0.9981388 & 79.90000 & 23.63070 & -60.62500\end{array}$

$\begin{array}{lllllll}505 & 13235.8369 & 13195.3447 & 0.9969407 & 75.47649 & 23.63070 & -55.00000\end{array}$

$\begin{array}{lllllll}506 & 15801.0771 & 15724.3154 & 0.9951420 & 71.05299 & 23.63070 & -55.00000\end{array}$

$\begin{array}{lllllll}507 & 21384.1797 & 21374.8691 & 0.9995646 & 71.05299 & 23.63070 & -60.62500\end{array}$

$\begin{array}{llllllll}508 & 15424.8838 & 15418.3398 & 0.9995757 & 75.47649 & 23.63070 & -60.62500\end{array}$

$\begin{array}{lllllll}511 & 7406.6182 & 6829.8491 & 0.9221279 & 15.25000 & -9.64501 & -66.25000\end{array}$

$\begin{array}{lllllll}524 & 9620.8887 & 10108.7266 & 1.0507061 & 28.26050 & -9.64501 & -66.25000\end{array}$

$\begin{array}{lllllll}538 & 6601.4473 & 7869.1362 & 1.1920320 & 15.25000 & -9.64501 & -77.03100\end{array}$

$\begin{array}{lllllll}539 & 9114.5664 & 6595.8481 & 0.7236601 & 15.25000 & -9.64501 & -71.64000\end{array}$

$\begin{array}{lllllll}542 & 6885.4316 & 6373.0234 & 0.9255808 & 19.58683 & -9.64501 & -66.25000\end{array}$

$\begin{array}{llllllll}543 & 7686.2979 & 8013.3911 & 1.0425553 & 23.92366 & -9.64501 & -66.25000\end{array}$

$\begin{array}{lllllll}544 & 7939.5073 & 6806.8804 & 0.8573429 & 28.26050 & -9.64501 & -77.03100\end{array}$

$\begin{array}{llllllll}545 & 10971.8213 & 12951.3438 & 1.1804187 & 28.26050 & -9.64501 & -71.64000\end{array}$

$\begin{array}{lllllll}546 & 11031.4912 & 15168.6348 & 1.3750303 & 23.92366 & -9.64501 & -77.03100\end{array}$

$\begin{array}{llllllll}547 & 11312.3691 & 6459.8115 & 0.5710397 & 19.58683 & -9.64501 & -77.03100\end{array}$

$\begin{array}{llllllll}548 & 14395.0352 & 9077.9609 & 0.6306314 & 19.58683 & -9.64501 & -71.64000\end{array}$

$\begin{array}{lllllll}549 & 14264.2383 & 19345.8613 & 1.3562492 & 23.92366 & -9.64501 & -71.64000\end{array}$ 
NODE STATIC FAILURE FACTOR $X$-LOC Y-LOC ZLOC

$1038 \quad 14083.2598 \quad 14080.2090 \quad 0.9997834 \quad-66.62949 \quad 23.63070 \quad-5.62500$

$\begin{array}{lllllll}1100 & 4698.7598 & 4710.0820 & 1.0024096 & -79.90000 & 23.63070 & 0.00000\end{array}$

$\begin{array}{lllllll}1101 & 8676.7129 & 8682.2637 & 1.0006397 & -71.05299 & 23.63070 & 0.00000\end{array}$

$\begin{array}{lllllll}1102 & 7697.5327 & 7721.4653 & 1.0031091 & -75.47649 & 23.63070 & 0.00000\end{array}$

$\begin{array}{lllllll}1103 & 6581.2065 & 6583.8311 & 1.0003988 & -79.90000 & 23.63070 & -11.25000\end{array}$

$\begin{array}{lllllll}1104 & 7036.5752 & 7028.6108 & 0.9988682 & -79.90000 & 23.63070 & -5.62500\end{array}$

$\begin{array}{lllllll}1105 & 13235.8369 & 13209.4824 & 0.9980088 & -75.47649 & 23.63070 & -11.25000\end{array}$

$\begin{array}{lllllll}1106 & 15801.0771 & 15752.3721 & 0.9969176 & -71.05299 & 23.63070 & -11.25000\end{array}$

$\begin{array}{llllllll}1107 & 21384.1797 & 21378.9414 & 0.9997550 & -71.05299 & 23.63070 & -5.62500\end{array}$

$\begin{array}{llllllll}1108 & 15424.8838 & 15420.9531 & 0.99974452 & -75.47649 & 23.63070 & -5.62500\end{array}$

$\begin{array}{lllllll}1111 & 7406.6182 & 7360.6636 & 0.9937955 & -15.25000 & -9.64501 & 0.00000\end{array}$

$\begin{array}{lllllll}1124 & 9620.8887 & 9865.5557 & 1.0254308 & -28.26050 & -9.64501 & 0.00000\end{array}$

$\begin{array}{lllllll}1138 & 6601.4473 & 6270.7925 & 0.9499118 & -15.25000 & -9.64501 & 10.78100\end{array}$

$\begin{array}{lllllll}1139 & 9114.5664 & 8953.5605 & 0.9823353 & -15.25000 & -9.64501 & 5.39050\end{array}$

$\begin{array}{lllllll}1142 & 6885.4316 & 7024.0957 & 1.0201387 & -19.58683 & -9.64501 & 0.00000\end{array}$

$\begin{array}{lllllll}1143 & 7686.2979 & 7943.1948 & 1.0334227 & -23.92366 & -9.64501 & 0.00000\end{array}$

$\begin{array}{lllllll}1144 & 7939.5073 & 8230.5752 & 1.0366607 & -28.26050 & -9.64501 & 10.78100\end{array}$

$\begin{array}{lllllll}1145 & 10971.8213 & 11172.3926 & 1.0182806 & -28.26050 & -9.64501 & 5.39050\end{array}$

$\begin{array}{lllllll}1146 & 11031.4912 & 11025.5322 & 0.9994598 & -23.92366 & -9.64501 & 10.78100\end{array}$

$\begin{array}{lllllll}1147 & 11312.3691 & 11353.0723 & 1.0035981 & -19.58683 & -9.64501 & 10.78100\end{array}$

$\begin{array}{lllllll}1148 & 14395.0352 & 14388.4805 & 0.9995447 & -19.58683 & -9.64501 & 5.39050\end{array}$

$\begin{array}{lllllll}1149 & 14264.2383 & 14286.6270 & 1.0015695 & -23.92366 & -9.64501 & 5.39050\end{array}$ 
NODE STATIC FAILURE FACTOR X-LOC Y-LOC ZLOC

$\begin{array}{lllllll}1438 & 14083.2598 & 14086.7324 & 1.0002465 & -66.62949 & 23.63070 & -60.62500\end{array}$

$\begin{array}{lllllll}1500 & 4698.7598 & 4686.5581 & 0.9974032 & -79.90000 & 23.63070 & -66.25000\end{array}$

$\begin{array}{lllllll}1501 & 8676.7129 & 8672.0547 & 0.9994631 & -71.05299 & 23.63070 & -66.25000\end{array}$

$\begin{array}{lllllll}1502 & 7697.5327 & 7671.1323 & 0.9965703 & -75.47649 & 23.63070 & -66.25000\end{array}$

$\begin{array}{lllllll}1503 & 6581.2065 & 6578.4258 & 0.9995775 & -79.90000 & 23.63070 & -55.00000\end{array}$

$\begin{array}{lllllll}1504 & 7036.5752 & 7046.0713 & 1.0013496 & -79.90000 & 23.63070 & -60.62500\end{array}$

$\begin{array}{lllllll}1505 & 13235.8369 & 13264.9941 & 1.0022029 & -75.47649 & 23.63070 & -55.00000\end{array}$

$\begin{array}{lllllll}1506 & 15801.0771 & 15855.4746 & 1.0034426 & -71.05299 & 23.63070 & -55.00000\end{array}$

$\begin{array}{lllllll}1507 & 21384.1797 & 21390.4980 & 1.0002955 & -71.05299 & 23.63070 & -60.62500\end{array}$

$\begin{array}{lllllll}1508 & 15424.8838 & 15429.4551 & 1.0002964 & -75.47649 & 23.63070 & -60.62500\end{array}$

$\begin{array}{lllllll}1511 & 7406.6182 & 7730.1143 & 1.0436766 & -15.25000 & -9.64501 & -66.25000\end{array}$

$\begin{array}{lllllll}1524 & 9620.8887 & 9492.4131 & 0.9866462 & -28.26050 & -9.64501 & -66.25000\end{array}$

$\begin{array}{lllllll}1538 & 6601.4473 & 7348.6641 & 1.1131898 & -15.25000 & -9.64501 & -77.03100\end{array}$

$\begin{array}{lllllll}1539 & 9114.5664 & 9908.8457 & 1.0871439 & -15.25000 & -9.64501 & -71.64000\end{array}$

$\begin{array}{lllllll}1542 & 68885.4316 & 6773.4512 & 0.9837366 & -19.58683 & -9.64501 & -66.25000\end{array}$

$\begin{array}{lllllll}1543 & 7686.2979 & 7438.9023 & 0.9678134 & -23.92366 & -9.64501 & -66.25000\end{array}$

$\begin{array}{lllllll}1544 & 7939.5073 & 7928.6328 & 0.9986303 & -28.26050 & -9.64501 & -77.03100\end{array}$

$1545 \quad 10971.8213 \quad 11131.9619 \quad 1.0145956 \quad-28.26050 \quad-9.64501 \quad-71.64000$

$\begin{array}{lllllll}1546 & 11031.4912 & 11568.7227 & 1.0486999 & -23.92366 & -9.64501 & -77.03100\end{array}$

$\begin{array}{lllllll}1547 & 11312.3691 & 11759.5215 & 1.0395278 & -19.58683 & -9.64501 & -77.03100\end{array}$

$\begin{array}{lllllll}1548 & 14395.0352 & 15046.9102 & 1.0452847 & -19.58683 & -9.64501 & -71.64000\end{array}$

$\begin{array}{llllllll}1549 & 14264.2383 & 14932.9971 & 1.0468836 & -23.92366 & -9.64501 & -71.64000\end{array}$ 


\section{APPENDDX 4}

NUMBERT WO

FACTOR LISTING 


\section{NUMBERTWO WORST}

NODE STATIC FAILURE FACTOR $X$-LOC Y-LOC ZLOC

$119 \quad 6534.1294 \quad 4695.2827 \quad 0.7185782 \quad 15.25000-13.94750 \quad 0.00000$

$\begin{array}{lllllll}139 & 5000.1997 & 3747.8337 & 0.7495368 & 15.25000 & -9.64501 & 5.39050\end{array}$

$141 \quad 5901.5444 \quad 4250.4063 \quad 0.7202193 \quad 15.24870 \quad-4.27001 \quad 5.39050$

$\begin{array}{llllllll}142 & 3779.1233 & 2749.6501 & 0.7275894 & 19.58683 & -9.64501 & 0.00000\end{array}$

$\begin{array}{llllllll}145 & 4577.7803 & 5909.7163 & 1.2909567 & 28.26050 & -9.64501 & 5.39050\end{array}$

$\begin{array}{lllllll}152 & 4057.0024 & 5014.3447 & 1.2359728 & 28.26047 & -0.65095 & 5.39050\end{array}$

$\begin{array}{llllllll}153 & 6224.6641 & 8184.3057 & 1.3148189 & 28.26047 & -5.14798 & 5.39050\end{array}$

$\begin{array}{lllllll}188 & 7970.8433 & 5656.6582 & 0.7096688 & 15.24827 & -2.47834 & 0.00000\end{array}$

$\begin{array}{llllllll}189 & 2013.9762 & 2525.1011 & 1.2537889 & 15.24913 & -6.06167 & 0.00000\end{array}$

$\begin{array}{lllllll}194 & 6526.2607 & 4742.6914 & 0.7267088 & 23.93646 & -5.51314 & 0.00000\end{array}$

$\begin{array}{llllllll}195 & 4778.5171 & 3263.9058 & 0.6830374 & 19.59871 & -5.81784 & 0.00000\end{array}$

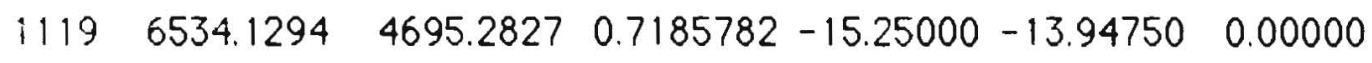

$\begin{array}{lllllll}7139 & 5000.1997 & 3747.8337 & 0.7495368 & -15.25000 & -9.64501 & 5.39050\end{array}$

$1141 \quad 5901.5444 \quad 4250.4063 \quad 0.7202193 \quad-15.24870 \quad-4.27001 \quad 5.39050$

$\begin{array}{lllllll}1142 & 3779.1233 & 2749.6501 & 0.7275894 & -19.58683 & -9.64501 & 0.00000\end{array}$

$\begin{array}{lllllll}1145 & 4577.7803 & 5909.7163 & 1.2909567 & -28.26050 & -9.64501 & 5.39050\end{array}$

$\begin{array}{lllllll}1152 & 4057.0024 & 5014.3447 & 1.2359728 & -28.26047 & -0.65095 & 5.39050\end{array}$

$\begin{array}{lllllll}1153 & 6224.6641 & 8184.3057 & 1.3148189 & -28.26047 & -5.14798 & 5.39050\end{array}$

$\begin{array}{lllllll}1188 & 7970.8433 & 5656.6582 & 0.7096688 & -15.24827 & -2.47834 & 0.00000\end{array}$

$\begin{array}{lllllll}1189 & 2013.9762 & 2525.1011 & 1.2537889 & -15.24913 & -6.06167 & 0.00000\end{array}$

$\begin{array}{lllllll}1194 & 6526.2607 & 4742.6914 & 0.7267088 & -23.93646 & -5.51314 & 0.00000\end{array}$

$\begin{array}{llllllll}1195 & 4778.5171 & 3263.9058 & 0.6830374 & -19.59871 & -5.81784 & 0.00000\end{array}$ 


\section{SECTION A-A}

\section{QUADRANT ।}

$\begin{array}{rrrrrrrr}\text { NODE } & \text { STATIC } & \text { FAILURE } & \text { FACTOR } & \text { X-LOC } & \text { Y-LOC } & \text { ZLOC } \\ 14 & 11704.3320 & 10917.6123 & 0.9327839 & 19.62247 & 1.83647 & 0.00000 \\ 15 & 9258.9902 & 9197.2881 & 0.9933360 & 23.96206 & 2.75057 & 0.00000 \\ 113 & 6400.1460 & 5148.7349 & 0.8044715 & 15.24870 & -4.27001 & 0.00000 \\ 126 & 5711.4658 & 5942.8662 & 1.0405151 & 28.26048 & -5.14798 & 0.00000 \\ 127 & 5161.3184 & 5433.3174 & 1.0526996 & 28.26047 & -0.65095 & 0.00000 \\ 142 & 3779.1233 & 2749.6501 & 0.7275894 & 19.58683 & -9.64501 & 0.00000 \\ 143 & 6404.4614 & 5235.0938 & 0.8174136 & 23.92366 & -9.64501 & 0.00000 \\ 188 & 7970.8433 & 5656.6582 & 0.7096688 & 15.24827 & -2.47834 & 0.00000 \\ 189 & 2013.9762 & 2525.1011 & 1.2537889 & 15.24913 & -6.06167 & 0.00000\end{array}$

QUADRANT 2

NODE STATIC FAILURE FACTOR X-LOC Y-LOC ZLOC

$\begin{array}{lllllll}414 & \overline{1} 1704.3320 & 11680.6465 & 0.9979764 & 19.62247 & 1.83647 & -66.25000\end{array}$

$\begin{array}{lllllll}415 & 9258.9902 & 9243.0996 & 0.9982837 & 23.96206 & 2.75057 & -66.25000\end{array}$

$\begin{array}{lllllll}513 & 6400.1460 & 6413.4307 & 1.0020757 & 15.24870 & -4.27001 & -66.25000\end{array}$

$\begin{array}{lllllll}526 & 5711.4658 & 5699.2935 & 0.9978688 & 28.26048 & -5.14798 & -66.25000\end{array}$

$\begin{array}{lllllll}527 & 5161.3184 & 5150.6313 & 0.9979294 & 28.26047 & -0.65095 & -66.25000\end{array}$

$\begin{array}{lllllll}542 & 3779.1233 & 3763.7559 & 0.9959336 & 19.58683 & -9.64501 & -66.25000\end{array}$

$\begin{array}{lllllll}543 & 6404.4609 & 6380.1396 & 0.9962025 & 23.92366 & -9.64501 & -66.25000\end{array}$

$\begin{array}{lllllll}588 & 7970.8433 & 7936.1641 & 0.9956492 & 15.24827 & -2.47834 & -66.25000\end{array}$

$\begin{array}{lllllll}589 & 2013.9762 & 2030.7102 & 1.0083089 & 15.24913 & -6.06167 & -66.25000\end{array}$ 


\section{QUADRANT 3}

NODE STATIC FAILURE FACTOR X-LOC Y-LOC ZLOC

$\begin{array}{lllllll}1014 & 11704.3320 & 10917.6123 & 0.9327839 & -19.62247 & 1.83647 & 0.00000\end{array}$

$\begin{array}{lllllll}1015 & 9258.9902 & 9197.2881 & 0.9933360 & -23.96206 & 2.75057 & 0.00000\end{array}$

$\begin{array}{lllllll}1113 & 6400.1460 & 5148.7349 & 0.8044715 & -15.24870 & -4.27001 & 0.00000\end{array}$

$\begin{array}{lllllll}1126 & 5711.4658 & 5942.8662 & 1.0405151 & -28.26048 & -5.14798 & 0.00000\end{array}$

$\begin{array}{lllllll}1127 & 5161.3184 & 5433.3174 & 1.0526996 & -28.26047 & -0.65095 & 0.00000\end{array}$

$\begin{array}{lllllll}1142 & 3779.1233 & 2749.6501 & 0.7275894 & -19.58683 & -9.64501 & 0.00000\end{array}$

$\begin{array}{lllllll}1143 & 6404.4614 & 5235.0938 & 0.8174136 & -23.92366 & -9.64501 & 0.00000\end{array}$

$\begin{array}{lllllll}1188 & 7970.8433 & 5656.6582 & 0.7096688 & -15.24827 & -2.47834 & 0.00000\end{array}$

$\begin{array}{lllllll}1189 & 2013.9762 & 2525.1011 & 1.2537889 & -15.24913 & -6.06167 & 0.00000\end{array}$

\section{QUADRANT 4}

NODE STATIC FAILURE FACTOR X-LOC Y-LOC ZLOC

$\begin{array}{llllllll}1414 & 11704.332 & 11680.6465 & 0.9979764 & -19.62247 & 1.83647 & -66.25\end{array}$

$\begin{array}{lllllll}1415 & 9258.9902 & 9243.0996 & 0.9982837 & -23.96206 & 2.75057 & -66.25\end{array}$

$\begin{array}{lllllll}1513 & 6400.1460 & 6413.4307 & 1.0020757 & -15.24870 & -4.27001 & -66.25000\end{array}$

$\begin{array}{lllllll}1526 & 5711.4658 & 5699.2935 & 0.9978688 & -28.26048 & -5.14798 & -66.25000\end{array}$

$\begin{array}{lllllll}1527 & 5161.3184 & 5150.6313 & 0.9979294 & -28.26047 & -0.65095 & -66.25000\end{array}$

$\begin{array}{lllllll}1542 & 3779.1233 & 3763.7559 & 0.9959336 & -19.58683 & -9.64501 & -66.25000\end{array}$

$\begin{array}{llllllll}1543 & 6404.4609 & 6380.1396 & 0.9962025 & -23.92366 & -9.64501 & -66.25000\end{array}$

$\begin{array}{lllllll}1588 & 7970.8433 & 7936.1641 & 0.9956492 & -15.24827 & -2.47834 & -66.25000\end{array}$

$\begin{array}{lllllll}1589 & 2013.9762 & 2030.7102 & 1.0083089 & -15.24913 & -6.06167 & -66.25000\end{array}$ 


\section{SECTION B-B}

NODE STATIC FAILURE FACTOR X-LOC Y-LOC ZLOC

$\begin{array}{lllllll}75 & 1145.4335 & 1124.5837 & 0.9817975 & 15.24740 & 1.10498 & -33.12500\end{array}$

$\begin{array}{lllllll}78 & 1837.4819 & 1811.1801 & 0.9856859 & 0.41207 & 0.00000 & -33.12500\end{array}$

$\begin{array}{lllllll}79 & 519.1904 & 530.3132 & 1.0214232 & 10.18418 & 0.49153 & -33.12500\end{array}$

$\begin{array}{lllllll}80 & 1419.4530 & 1408.1710 & 0.9920519 & 5.09876 & 0.12299 & -33.12500\end{array}$

$\begin{array}{lllllll}87 & 4898.3145 & 4903.1714 & 1.0009916 & 28.26045 & 3.84607 & -33.12500\end{array}$

$90 \quad 3179.0029 \quad 3161.2742 \quad 0.9944232 \quad 23.96206 \quad 2.75057 \quad-33.12500$

$91 \quad 2120.7888 \quad 2094.3047 \quad 0.9875121 \quad 19.62247 \quad 1.83647 \quad-33.12500$

$\begin{array}{lllllll}96 & 6835.5942 & 6858.2622 & 1.0033162 & 31.97561 & 4.95008 & -33.12500\end{array}$

$\begin{array}{lllllll}216 & 937.4582 & 904.4899 & 0.9648323 & 15.25000 & -18.25000 & -33.12500\end{array}$

$\begin{array}{lllllll}219 & 2182.5669 & 2153.4124 & 0.9866421 & 0.00000 & -18.25000 & -33.12500\end{array}$

$\begin{array}{lllllll}220 & 1316.0605 & 1283.5681 & 0.9753109 & 10.16666 & -18.25000 & -33.12500\end{array}$

$221 \quad 1952.1735 \quad 1924.6079 \quad 0.9858795 \quad 5.08333-18.25000-33.12500$

$\begin{array}{lllllll}1075 & 1145.4335 & 1124.5837 & 0.9817975 & -15.24740 & 1.10498 & -33.12500\end{array}$

$1079 \quad 519.1904 \quad 530.3132 \quad 1.0214232-10.18418 \quad 0.49153 \quad-33.12500$

$\begin{array}{lllllll}1080 & 1419.4530 & 1408.1710 & 0.9920519 & -5.09876 & 0.12299 & -33.12500\end{array}$

$\begin{array}{lllllll}1087 & 4898.3145 & 4903.1714 & 1.0009916 & -28.26045 & 3.84607 & -33.12500\end{array}$

$\begin{array}{lllllll}1090 & 3179.0029 & 3161.2742 & 0.9944232 & -23.96206 & 2.75057 & -33.12500\end{array}$

$\begin{array}{llllllll}1091 & 2120.7888 & 2094.3047 & 0.9875121 & -19.62247 & 1.83647 & -33.12500\end{array}$

$\begin{array}{lllllll}1096 & 6835.5942 & 6858.2622 & 1.0033162 & -31.97561 & 4.95008 & -33.12500\end{array}$

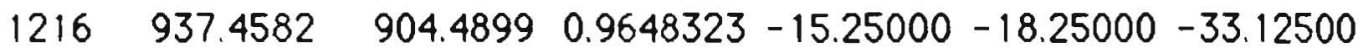

$\begin{array}{lllllll}1220 & 1316.0605 & 1283.5681 & 0.9753109 & -10.16666 & -18.25000 & -33.12500\end{array}$

$\begin{array}{lllllll}1221 & 1952.1735 & 1924.6079 & 0.9858795 & -5.08333 & -18.25000 & -33.12500\end{array}$

$1242 \quad 4850.7827 \quad 4819.7144 \quad 0.9935952-23.92366-18.25000 \quad-33.12500$

$1243 \quad 2281.9146 \quad 2253.0295 \quad 0.9873418-19.58683-18.25000-33.12500$ 


\section{QUADRANT 1}

NODE STATIC FAILURE FACTOR X-LOC Y-LOC ZLOC

$121 \quad 14662.2695 \quad 14116.0283 \quad 0.9627451 \quad 28.26050 \quad-9.64501 \quad-11.25000$

$\begin{array}{lllllll}124 & 9262.8857 & 9824.2871 & 1.0606076 & 28.26050 & -9.64501 & 0.00000\end{array}$

$125 \quad 5323.7173 \quad 5858.3745 \quad 1.1004293 \quad 28.26050 \quad-9.64501 \quad-5.62500$

$\begin{array}{lllllll}181 & 10540.7070 & 10246.0508 & 0.9720459 & 28.26050 & -18.25000 & 0.00000\end{array}$

$\begin{array}{lllllll}182 & 12551.1602 & 12615.9092 & 1.0051588 & 28.26050 & -13.94750 & 0.00000\end{array}$

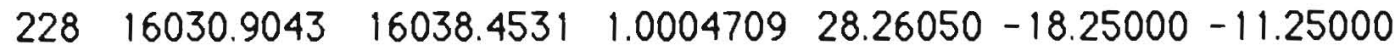

$\begin{array}{lllllll}231 & 8540.9580 & 8732.8896 & 1.0224719 & 28.26050 & -18.25000 & -5.62500\end{array}$

$\begin{array}{lllllll}282 & 19003.9219 & 18614.7031 & 0.9795190 & 28.26050 & -13.94750 & -11.25000\end{array}$

$\begin{array}{lllllll}305 & 6782.7661 & 7491.7134 & 1.1045219 & 28.26050 & -13.94750 & -5.62500\end{array}$

\section{QUADRANT 2}

NODE STATIC FAILURE FACTOR X-LOC-Y-LOC ZLOC

$\begin{array}{lllllll}521 & 14662.2695 & 14657.3818 & 0.9996666 & 28.26050 & -9.64501 & -55.00000\end{array}$

$\begin{array}{lllllll}524 & 9262.8857 & 9249.7090 & 0.9985775 & 28.26050 & -9.64501 & -66.25000\end{array}$

$\begin{array}{lllllll}525 & 5323.7173 & 5316.1890 & 0.9985859 & 28.26050 & -9.64501 & -60.62500\end{array}$

$\begin{array}{lllllll}581 & 10540.7070 & 10533.6426 & 0.9993298 & 28.26050 & -18.25000 & -66.25000\end{array}$

$\begin{array}{lllllll}582 & 12551.1602 & 12534.4990 & 0.9986725 & 28.26050 & -13.94750 & -66.25000\end{array}$

$\begin{array}{lllllll}628 & 16030.9043 & 15998.8115 & 0.9979981 & 28.26050 & -18.25000 & -55.00000\end{array}$

$\begin{array}{lllllll}631 & 8540.9580 & 8530.2480 & 0.9987460 & 28.26050 & -18.25000 & -60.62500\end{array}$

$\begin{array}{lllllll}682 & 19003.9219 & 18986.0566 & 0.9990599 & 28.26050 & -13.94750 & -55.00000\end{array}$

$\begin{array}{lllllll}705 & 6782.7661 & 6773.0342 & 0.9985652 & 28.26050 & -13.94750 & -60.62500\end{array}$ 


\section{QUADRANT 3}

NODE STATIC FAILURE FACTOR X-LOC Y-LOC ZLOC

$\begin{array}{lllllll}1121 & 14662.2695 & 14116.0283 & 0.9627451 & -28.26050 & -9.64501 & -11.25000\end{array}$

$\begin{array}{lllllll}1124 & 9262.8857 & 9824.2871 & 1.0606076 & -28.26050 & -9.64501 & 0.00000\end{array}$

$\begin{array}{lllllll}1125 & 5323.7173 & 5858.3745 & 1.1004293 & -28.26050 & -9.64501 & -5.62500\end{array}$

$\begin{array}{lllllll}1181 & 10540.7070 & 10246.0508 & 0.9720459 & -28.26050 & -18.25000 & 0.00000\end{array}$

$\begin{array}{lllllll}1182 & 12551.1602 & 12615.9092 & 1.0051588 & -28.26050 & -13.94750 & 0.00000\end{array}$

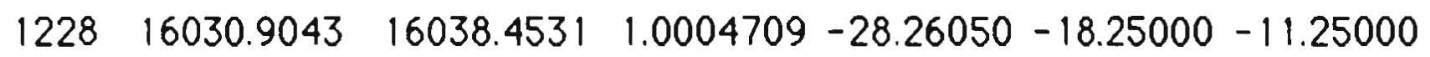

$1231 \quad 8540.9580 \quad 8732.8896 \quad 1.0224719-28.26050-18.25000 \quad-5.62500$

$\begin{array}{lllllll}1282 & 19003.9219 & 18614.7031 & 0.9795190 & -28.26050 & -13.94750 & -11.25000\end{array}$

$\begin{array}{lllllll}1305 & 6782.7661 & 7491.7134 & 1.1045219 & -28.26050 & -13.94750 & -5.62500\end{array}$

\section{QUADRANT 4}

NODE STATIC FAILURE FACTOR X-LOC Y-LOC ZLOC

$\begin{array}{lllllll}1521 & 14662.2695 & 14657.3818 & 0.99966666 & -28.26050 & -9.64501 & -55.00000\end{array}$

$\begin{array}{lllllll}1524 & 9262.8857 & 9249.7090 & 0.9985775 & -28.26050 & -9.64501 & -66.25000\end{array}$

$\begin{array}{lllllll}1525 & 5323.7173 & 5316.1890 & 0.9985859 & -28.26050 & -9.64501 & -60.62500\end{array}$

$\begin{array}{lllllll}1581 & 10540.7070 & 10533.6426 & 0.9993298 & -28.26050 & -18.25000 & -66.25000\end{array}$

$\begin{array}{lllllll}1582 & 12551.1602 & 12534.4990 & 0.9986725 & -28.26050 & -13.94750 & -66.25000\end{array}$

$\begin{array}{lllllll}1628 & 16030.9043 & 15998.8115 & 0.9979981 & -28.26050 & -18.25000 & -55.00000\end{array}$

$\begin{array}{lllllll}1631 & 8540.9580 & 8530.2480 & 0.9987460 & -28.26050 & -18.25000 & -60.62500\end{array}$

$\begin{array}{lllllll}1682 & 19003.9219 & 18986.0566 & 0.9990599 & -28.26050 & -13.94750 & -55.00000\end{array}$

$\begin{array}{lllllll}1705 & 6782.7661 & 6773.0342 & 0.9985652 & -28.26050 & -13.94750 & -60.62500\end{array}$ 


\section{TIE PLATE}

NODE STATIC FAILURE FACTOR X-LOC Y-LOC ZLOC

$1 \quad 18590.9414 \quad 17829.1992 \quad 0.9590262 \quad 0.41207 \quad 0.00000 \quad 0.00000$

$\begin{array}{lllllll}7 & 13989.6982 & 13451.2559 & 0.9615115 & 0.41207 & 0.00000 & -11.25000\end{array}$

$\begin{array}{lllllll}10 & 17322.6250 & 16646.3047 & 0.9609574 & 0.41207 & 0.00000 & -5.62500\end{array}$

$51 \quad 6976.9146 \quad 6680.1025 \quad 0.9574580 \quad 0.41207 \quad 0.00000-19.12500$

$\begin{array}{lllllll}54 & 10640.6738 & 10213.9355 & 0.9598956 & 0.41207 & 0.00000 & -15.18700\end{array}$

$\begin{array}{lllllll}78 & 1837.4819 & 1811.1801 & 0.9856859 & 0.41207 & 0.00000 & -33.12500\end{array}$

$\begin{array}{lllllll}81 & 1768.3112 & 1742.7345 & 0.9855361 & 0.41207 & 0.00000 & -28.45800\end{array}$

$\begin{array}{lllllll}82 & 3195.1631 & 3039.5500 & 0.9512973 & 0.41207 & 0.00000 & -23.79100\end{array}$

$\begin{array}{lllllll}201 & 15208.0459 & 14601.5918 & 0.9601228 & 0.00000 & -18.25000 & 0.00000\end{array}$

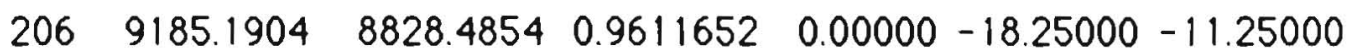

$\begin{array}{lllllll}209 & 12347.4111 & 11871.4229 & 0.9614503 & 0.00000 & -18.25000 & -5.62500\end{array}$

$\begin{array}{lllllll}212 & 6175,9893 & 5922.8037 & 0.9590049 & 0.00000 & -18.25000 & -16.62500\end{array}$

$\begin{array}{lllllll}219 & 2182.5669 & 2153.4124 & 0.9866421 & 0.00000 & -18.25000 & -33.12500\end{array}$

$\begin{array}{lllllll}222 & 2+46.0637 & 2098.5657 & 0.9778674 & 0.00000 & -18.25000 & -27.62500\end{array}$

$\begin{array}{lllllll}223 & 3621.4751 & 3454.3167 & 0.9538425 & 0.00000 & -18.25000 & -22.12500\end{array}$

$\begin{array}{lllllll}401 & 18590.9414 & 18601.1816 & 1.0005509 & 0.41207 & 0.00000 & -66.25000\end{array}$

$\begin{array}{lllllll}407 & 13989.6982 & 13961.0098 & 0.9979493 & 0.41207 & 0.00000 & -55.00000\end{array}$

$\begin{array}{lllllll}410 & 17322.6250 & 17302.0273 & 0.9988109 & 0.41207 & 0.00000 & -60.62500\end{array}$

$\begin{array}{lllllll}451 & 6976.9146 & 6965.3960 & 0.9983491 & 0.41207 & 0.00000 & -47.12500\end{array}$

$\begin{array}{lllllll}454 & 10640.6738 & 10617.7900 & 0.9978494 & 0.41207 & 0.00000 & -51.06200\end{array}$

$\begin{array}{lllllll}481 & 1768.3112 & 1742.7638 & 0.9855527 & 0.41207 & 0.00000 & -37.79100\end{array}$

$\begin{array}{lllllll}482 & 3195.1631 & 3196.3496 & 1.0003713 & 0.41207 & 0.00000 & -42.45800\end{array}$

$\begin{array}{lllllll}601 & 15208.0459 & 15224.8135 & 1.0011026 & 0.00000 & -18.25000 & -66.25000\end{array}$

$\begin{array}{lllllll}606 & 9185.1904 & 9187.8320 & 1.0002877 & 0.000 & -18.25 & -55.00\end{array}$

$\begin{array}{lllllll}609 & 12347.4111 & 12352.6768 & 1.0004264 & 0.000 & -18.25 & -60.625\end{array}$

$\begin{array}{lllllll}612 & 6175.9893 & 6175.7432 & 0.9999601 & 0.00000 & -18.25000 & -49.62500\end{array}$ 
$\begin{array}{llllllll}622 & 2146.0637 & 2116.6511 & 0.9862946 & 0.0000 & -18.25 & -38.625\end{array}$

$\begin{array}{lllllll}623 & 3621.4751 & 3612.3113 & 0.9974696 & 0.0000 & -18.25 & -44.125\end{array}$ 
APPENDDU 5
NUMBERTHREE

FACTOR LISTING 


\title{
STANCHION AND 3.5 IN SUPPORT PLATES
}

\author{
QUADRANT 1
}

NODE STATIC FAILURE FACTOR X-LOC Y-LOC ZLOC

$\begin{array}{lllllll}38 & 19647.1953 & 19647.3184 & 1.0000063 & 66.62949 & 23.63070 & -5.62500\end{array}$

$\begin{array}{lllllll}100 & 8247.0107 & 8236.5928 & 0.9987367 & 79.90000 & 23.63070 & 0.00000\end{array}$

$\begin{array}{lllllll}101 & 10987.7227 & 11005.5771 & 1.0016249 & 71.05299 & 23.63070 & 0.00000\end{array}$

$\begin{array}{lllllll}102 & 9593.8721 & 9604.1758 & 1.0010740 & 75.47649 & 23.63070 & 0.00000\end{array}$

$\begin{array}{lllllll}103 & 5315.9082 & 5311.6963 & 0.9992077 & 79.90000 & 23.63070 & -11.25000\end{array}$

$\begin{array}{llllllll}104 & 13225.1895 & 13221.5645 & 0.9997259 & 79.90000 & 23.63070 & -5.62500\end{array}$

$\begin{array}{lllllll}105 & 6819.0015 & 6804.6973 & 0.9979023 & 75.47649 & 23.63070 & -11.25000\end{array}$

$\begin{array}{lllllll}106 & 8967.4063 & 8973.6807 & 1.0006996 & 71.05299 & 23.63070 & -11.25000\end{array}$

$\begin{array}{lllllll}107 & 19577.0469 & 19578.4238 & 1.0000703 & 71.05299 & 23.63070 & -5.62500\end{array}$

$\begin{array}{lllllll}108 & 14296.0996 & 14296.4180 & 1.0000223 & 75.47649 & 23.63070 & -5.62500\end{array}$

$\begin{array}{lllllll}111 & 8474.1807 & 7096.0234 & 0.8373699 & 15.25000 & -9.64501 & 0.00000\end{array}$

$\begin{array}{lllllll}124 & 11943.5117 & 12422.1914 & 1.0400786 & 28.26050 & -9.64501 & 0.00000\end{array}$

$\begin{array}{lllllll}138 & 2833.5259 & 3569.9216 & 1.2598867 & 15.25000 & -9.64501 & 10.78100\end{array}$

$\begin{array}{lllllll}139 & 6096.6338 & 5121.8384 & 0.8401092 & 15.25000 & -9.64501 & 5.39050\end{array}$

$\begin{array}{lllllll}142 & 9376.6250 & 8290.0439 & 0.8841181 & 19.58683 & -9.64501 & 0.00000\end{array}$

$\begin{array}{llllllll}143 & 10627.6641 & 11998.2119 & 1.1289604 & 23.92366 & -9.64501 & 0.00000\end{array}$

$\begin{array}{llllllll}144 & 2313.8179 & 2638.8293 & 1.1404655 & 28.26050 & -9.64501 & 10.78100\end{array}$

$\begin{array}{lllllll}145 & 6125.6860 & 7169.2417 & 1.1703573 & 28.26050 & -9.64501 & 5.39050\end{array}$

$\begin{array}{lllllll}146 & 12254.0566 & 17151.2793 & 1.3996409 & 23.92366 & -9.64501 & 10.78100\end{array}$

$\begin{array}{lllllll}147 & 12552.8975 & 7952.0659 & 0.6334845 & 19.58683 & -9.64501 & 10.78100\end{array}$

$\begin{array}{lllllll}148 & 14107.5088 & 9657.8145 & 0.6845868 & 19.58683 & -9.64501 & 5.39050\end{array}$

$\begin{array}{lllllll}149 & 13893.0703 & 19624.5352 & 1.4125413 & 23.92366 & -9.64501 & 5.39050\end{array}$ 


\section{QUADRANT $\mathbf{z}$}

NODE STATIC FAILURE FACTOR $X$-LOC Y-LOC ZLOC

$\begin{array}{lllllll}438 & 19647.1953 & 19647.1289 & 0.9999966 & 66.62949 & 23.63070 & -60.62500\end{array}$

$\begin{array}{lllllll}500 & 8247.0107 & 8248.8594 & 1.0002241 & 79.90000 & 23.63070 & -66.25000\end{array}$

$\begin{array}{lllllll}501 & 10987.7227 & 10984.3555 & 0.9996936 & 71.05299 & 23.63070 & -66.25000\end{array}$

$\begin{array}{lllllll}502 & 9593.8721 & 9591.9902 & 0.9998038 & 75.47649 & 23.63070 & -66.25000\end{array}$

$\begin{array}{lllllll}503 & 5315.9082 & 5316.7061 & 1.0001501 & 79.90000 & 23.63070 & -55.00000\end{array}$

$\begin{array}{lllllll}504 & 13225.1895 & 13225.8604 & 1.0000508 & 79.90000 & 23.63070 & -60.62500\end{array}$

$\begin{array}{lllllll}505 & 6819.0015 & 6821.5044 & 1.0003670 & 75.47649 & 23.63070 & -55.00000\end{array}$

$\begin{array}{lllllll}506 & 8967.4063 & 8966.5879 & 0.9999087 & 71.05299 & 23.63070 & -55.00000\end{array}$

$\begin{array}{lllllll}507 & 19577.0469 & 19576.7637 & 0.9999855 & 71.05299 & 23.63070 & -60.62500\end{array}$

$\begin{array}{llllllll}508 & 14296.0996 & 14296.0195 & 0.9999944 & 75.47649 & 23.63070 & -60.62500\end{array}$

$\begin{array}{lllllll}511 & 8474.1807 & 8484.9297 & 1.0012684 & 15.25000 & -9.64501 & -66.25000\end{array}$

$\begin{array}{lllllll}524 & 11943.5117 & 11923.8584 & 0.9983545 & 28.26050 & -9.64501 & -66.25000\end{array}$

$\begin{array}{llllllll}538 & 2833.5259 & 2833.8892 & 1.0001281 & 15.25000 & -9.64501 & -77.03100\end{array}$

$\begin{array}{lllllll}539 & 6096.6338 & 6092.6724 & 0.9993502 & 15.25000 & -9.64501 & -71.64000\end{array}$

$\begin{array}{lllllll}542 & 9376.6250 & 9374.9619 & 0.9998226 & 19.58683 & -9.64501 & -66.25000\end{array}$

$\begin{array}{lllllll}543 & 10627.6641 & 10618.3945 & 0.9991278 & 23.92366 & -9.64501 & -66.25000\end{array}$

$\begin{array}{lllllll}544 & 2313.8179 & 2305.5981 & 0.9964476 & 28.26050 & -9.64501 & -77.03100\end{array}$

$\begin{array}{lllllll}545 & 6125.6860 & 6114.8623 & 0.9982331 & 28.26050 & -9.64501 & -71.64000\end{array}$

$\begin{array}{lllllll}546 & 12254.0566 & 12254.7988 & 1.0000606 & 23.92366 & -9.64501 & -77.03100\end{array}$

$\begin{array}{lllllll}547 & 12552.8975 & 12551.1973 & 0.9998646 & 19.58683 & -9.64501 & -77.03100\end{array}$

$\begin{array}{llllllll}548 & 14107.5088 & 14107.9824 & 1.0000336 & 19.58683 & -9.64501 & -71.64000\end{array}$

$\begin{array}{llllllll}549 & 13893.0703 & 13891.7881 & 0.9999077 & 23.92366 & -9.64501 & -71.64000\end{array}$ 


\section{QUADRANT 3}

NODE STATIC FAILURE FACTOR X-LOC Y-LOC ZLOC

$\begin{array}{lllllll}1038 & 19647.1953 & 19647.3184 & 1.0000063 & -66.62949 & 23.63070 & -5.62500\end{array}$

$\begin{array}{lllllll}1100 & 8247.0107 & 8236.5928 & 0.9987367 & -79.90000 & 23.63070 & 0.00000\end{array}$

$\begin{array}{lllllll}1101 & 10987.7227 & 11005.5771 & 1.0016249 & -71.05299 & 23.63070 & 0.00000\end{array}$

$\begin{array}{lllllll}1102 & 9593.8721 & 9604.1758 & 1.0010740 & -75.47649 & 23.63070 & 0.00000\end{array}$

$\begin{array}{lllllll}1103 & 5315.9082 & 5311.6963 & 0.9992077 & -79.90000 & 23.63070 & -11.25000\end{array}$

$\begin{array}{lllllll}1104 & 13225.1895 & 13221.5645 & 0.9997259 & -79.90000 & 23.63070 & -5.62500\end{array}$

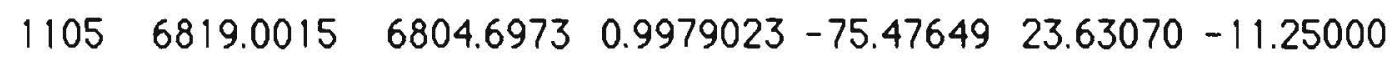

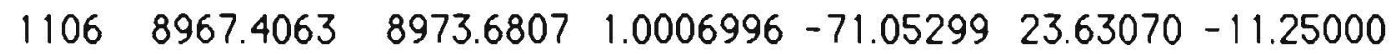

$\begin{array}{lllllll}1107 & 19577.0469 & 19578.4238 & 1.0000703 & -71.05299 & 23.63070 & -5.62500\end{array}$

$\begin{array}{lllllll}1108 & 14296.0996 & 14296.4180 & 1.0000223 & -75.47649 & 23.63070 & -5.62500\end{array}$

$\begin{array}{lllllll}1111 & 8474.1807 & 7096.0234 & 0.8373699 & -15.25000 & -9.64501 & 0.00000\end{array}$

$\begin{array}{lllllll}1124 & 11943.5117 & 12422.1914 & 1.0400786 & -28.26050 & -9.64501 & 0.00000\end{array}$

$\begin{array}{lllllll}1138 & 2833.5259 & 3569.9216 & 1.2598867 & -15.25000 & -9.64501 & 10.78100\end{array}$

$\begin{array}{lllllll}1139 & 6096.6338 & 5121.8384 & 0.8401092 & -15.25000 & -9.64501 & 5.39050\end{array}$

$\begin{array}{lllllll}1142 & 9376.6250 & 8290.0439 & 0.8841181 & -19.58683 & -9.64501 & 0.00000\end{array}$

$\begin{array}{lllllll}1143 & 10627.6641 & 11998.2119 & 1.1289604 & -23.92366 & -9.64501 & 0.00000\end{array}$

$\begin{array}{llllllll}1144 & 2313.8179 & 2638.8293 & 1.1404655 & -28.26050 & -9.64501 & 10.78100\end{array}$

$\begin{array}{lllllll}1145 & 6125.6860 & 7169.2417 & 1.1703573 & -28.26050 & -9.64501 & 5.39050\end{array}$

$\begin{array}{lllllll}1146 & 12254.0566 & 17151.2793 & 1.3996409 & -23.92366 & -9.64501 & 10.78100\end{array}$

$\begin{array}{lllllll}1147 & 12552.8975 & 7952.0659 & 0.6334845 & -19.58683 & -9.64501 & 10.78100\end{array}$

$\begin{array}{lllllll}1148 & 14107.5088 & 9657.8145 & 0.6845868 & -19.58683 & -9.64501 & 5.39050\end{array}$

$\begin{array}{lllllll}1149 & 13893.0703 & 19624.5352 & 1.4125413 & -23.92366 & -9.64501 & 5.39050\end{array}$ 


\section{QUADRANT 4}

NODE STATIC FAILURE FACTOR X-LOC Y-LOC ZLOC

$\begin{array}{lllllll}1438 & 19647.1953 & 19647.1289 & 0.9999966 & -66.62949 & 23.63070 & -60.62500\end{array}$

$\begin{array}{lllllll}1500 & 8247.0107 & 8248.8594 & 1.0002241 & -79.90000 & 23.63070 & -66.25000\end{array}$

$\begin{array}{lllllll}1501 & 10987.7227 & 10984.3555 & 0.9996936 & -71.05299 & 23.63070 & -66.25000\end{array}$

$\begin{array}{lllllll}1502 & 9593.8721 & 9591.9902 & 0.9998038 & -75.47649 & 23.63070 & -66.25000\end{array}$

$\begin{array}{lllllll}1503 & 5315.9082 & 5316.7061 & 1.0001501 & -79.90000 & 23.63070 & -55.00000\end{array}$

$\begin{array}{lllllll}1504 & 13225.1895 & 13225.8604 & 1.0000508 & -79.90000 & 23.63070 & -60.62500\end{array}$

$\begin{array}{lllllll}1505 & 6819.0015 & 6821.5044 & 1.0003670 & -75.47649 & 23.63070 & -55.00000\end{array}$

$\begin{array}{lllllll}1506 & 8967.4063 & 8966.5879 & 0.9999087 & -71.05299 & 23.63070 & -55.00000\end{array}$

$\begin{array}{lllllll}1507 & 19577.0469 & 19576.7637 & 0.9999855 & -71.05299 & 23.63070 & -60.62500\end{array}$

$\begin{array}{lllllll}1508 & 14296.0996 & 14296.0195 & 0.9999944 & -75.47649 & 23.63070 & -60.62500\end{array}$

$\begin{array}{lllllll}1511 & 8474.1807 & 8484.9297 & 1.0012684 & -15.25000 & -9.64501 & -66.25000\end{array}$

$\begin{array}{lllllll}1524 & 11943.5117 & 11923.8584 & 0.9983545 & -28.26050 & -9.64501 & -66.25000\end{array}$

$\begin{array}{lllllll}1538 & 2833.5259 & 2833.8892 & 1.0001281 & -15.25000 & -9.64501 & -77.03100\end{array}$

$1539 \quad 6096.6338 \quad 6092.6724 \quad 0.9993502-15.25000-9.64501-71.64000$

$\begin{array}{lllllll}1542 & 9376.6250 & 9374.9619 & 0.9998226 & -19.58683 & -9.64501 & -66.25000\end{array}$

$\begin{array}{lllllll}1543 & 10627.6641 & 10618.3945 & 0.9991278 & -23.92366 & -9.64501 & -66.25000\end{array}$

$\begin{array}{lllllll}1544 & 2313.8179 & 2305.5981 & 0.9964476 & -28.26050 & -9.64501 & -77.03100\end{array}$

$1545 \quad 6125.6860 \quad 6114.8623 \quad 0.9982331-28.26050 \quad-9.64501-71.64000$

$\begin{array}{lllllll}1546 & 12254.0566 & 12254.7988 & 1.0000606 & -23.92366 & -9.64501 & -77.03100\end{array}$

$\begin{array}{lllllll}1547 & 12552.8975 & 12551.1973 & 0.9998646 & -19.58683 & -9.64501 & -77.03100\end{array}$

$\begin{array}{lllllll}1548 & 14107.5088 & 14107.9824 & 1.0000336 & -19.58683 & -9.64501 & -71.64000\end{array}$

$\begin{array}{lllllll}1549 & 13893.0703 & 13891.7881 & 0.9999077 & -23.92366 & -9.64501 & -71.64000\end{array}$ 
NUMBERTHREE WORST

$\begin{array}{ccccccc}\text { NODE } & \text { STATIC } & \text { FAILURE } & \text { FACTOR } & \text { X-LOC } & \text { Y-LOC } & \text { ZLOC } \\ 519 & 6534.1294 & 4821.9385 & 0.7379619 & 15.25000 & -13.94750 & -66.25000 \\ 534 & 539.0928 & 690.3599 & 1.2805957 & 19.58510 & 2.01868 & -77.03100 \\ 539 & 5000.1997 & 3611.5278 & 0.7222767 & 15.25000 & -9.64501 & -71.64000 \\ 541 & 5901.5444 & 4095.4136 & 0.6939563 & 15.24870 & -4.27001 & -71.64000 \\ 545 & 4577.7803 & 5808.2876 & 1.2688000 & 28.26050 & -9.64501 & -71.64000 \\ 552 & 4057.0024 & 4921.1367 & 1.2129983 & 28.26047 & -0.65095 & -71.64000 \\ 553 & 6224.6641 & 8029.9155 & 1.2900159 & 28.26047 & -5.14798 & -71.64000 \\ 595 & 4778.5171 & 3528.1873 & 0.7383435 & 19.59871 & -5.81784 & -66.25000\end{array}$




\section{SECTION A-A}

\section{QUADRANT I}

NODE STATIC FAILURE FACTOR X-LOC Y-LOC ZLOC

$\begin{array}{lllllll}14 & 11704.3320 & 11568.3838 & 0.9883848 & 19.62247 & 1.83647 & 0.00000\end{array}$

$\begin{array}{lllllll}15 & 9258.9902 & 9091.1211 & 0.9818696 & 23.96206 & 2.75057 & 0.00000\end{array}$

$113 \quad 6400.1460 \quad 6316.8682 \quad 0.9869881 \quad 15.24870 \quad-4.27001 \quad 0.00000$

$\begin{array}{lllllll}126 & 5711.4658 & 5545.5776 & 0.9709553 & 28.26048 & -5.14798 & 0.00000\end{array}$

$\begin{array}{lllllll}127 & 5161.3184 & 5028.5488 & 0.9742761 & 28.26047 & -0.65095 & 0.00000\end{array}$

$\begin{array}{llllllll}142 & 3779.1233 & 3754.7361 & 0.9935468 & 19.58683 & -9.64501 & 0.00000\end{array}$

$143 \quad 6404.4614 \quad 6262.7324 \quad 0.9778703 \quad 23.92366 \quad-9.64501 \quad 0.00000$

$\begin{array}{lllllll}188 & 7970.8433 & 7991.4873 & 1.0025899 & 15.24827 & -2.47834 & 0.00000\end{array}$

$\begin{array}{llllllll}189 & 2013.9762 & 1965.2789 & 0.9758204 & 15.24913 & -6.06167 & 0.00000\end{array}$

\section{QUADRANT 2}

NODE STATIC FAILURE FACTOR X-LOC Y-LOC ZLOC $\begin{array}{lllllll}414 & 11704.3320 & 11422.1670 & 0.9758922 & 19.62247 & 1.83647 & -66.25000\end{array}$

$\begin{array}{lllllll}415 & 9258.9902 & 9364.8896 & 1.0114374 & 23.96206 & 2.75057 & -66.25000\end{array}$

$\begin{array}{llllllll}513 & 6400.1460 & 4936.4824 & 0.7713078 & 15.24870 & -4.27001 & -66.25000\end{array}$

$\begin{array}{lllllll}526 & 5711.4658 & 6046.2788 & 1.0586212 & 28.26048 & -5.14798 & -66.25000\end{array}$

$\begin{array}{llllllll}527 & 5161.3184 & 5497.0283 & 1.0650434 & 28.26047 & -0.65095 & -66.25000\end{array}$

$\begin{array}{lllllll}542 & 3779.1233 & 2947.3413 & 0.7799008 & 19.58683 & -9.64501 & -66.25000\end{array}$

$\begin{array}{lllllll}543 & 6404.4609 & 5563.5586 & 0.8687005 & 23.92366 & -9.64501 & -66.25000\end{array}$

$\begin{array}{lllllll}588 & 7970.8433 & 6378.8081 & 0.8002676 & 15.24827 & -2.47834 & -66.25000\end{array}$

$\begin{array}{lllllll}589 & 2013.9762 & 2151.2693 & 1.0681702 & 15.24913 & -6.06167 & -66.25000\end{array}$ 
NODE STATIC FAILURE FACTOR X-LOC Y-LOC ZLOC

$\begin{array}{lllllll}1014 & 11704.3320 & 11816.8047 & 1.0096095 & -19.62247 & 1.83647 & 0.00000\end{array}$

$\begin{array}{lllllll}1015 & 9258.9902 & 9410.9697 & 1.0164143 & -23.96206 & 2.75057 & 0.00000\end{array}$

$11136400.1460 \quad 6496.3145 \quad 1.0150260-15.24870-4.27001 \quad 0.00000$

$\begin{array}{lllllll}1126 & 5711.4658 & 5867.0444 & 1.0272397 & -28.26048 & -5.14798 & 0.00000\end{array}$

$\begin{array}{lllllll}1127 & 5161.3184 & 5283.7036 & 1.0237120 & -28.26047 & -0.65095 & 0.00000\end{array}$

$\begin{array}{lllllll}1142 & 3779.1233 & 3789.9307 & 1.0028597 & -19.58683 & -9.64501 & 0.00000\end{array}$

$\begin{array}{lllllll}1143 & 6404.4614 & 6523.7998 & 1.0186336 & -23.92366 & -9.64501 & 0.00000\end{array}$

$\begin{array}{lllllll}1188 & 7970.8433 & 7917.8696 & 0.9933541 & -15.24827 & -2.47834 & 0.00000\end{array}$

$\begin{array}{lllllll}1189 & 2013.9762 & 2078.9578 & 1.0322653 & -15.24913 & -6.06167 & 0.00000\end{array}$

\section{QUADRANT 4}

NODE STATIC FAILURE FACTOR X-LOC Y-LOC ZLOC

$\begin{array}{llllllll}1414 & 11704.3320 & 11204.7324 & 0.9573150 & -19.62247 & 1.83647 & -66.25000\end{array}$

$\begin{array}{lllllll}1415 & 9258.9902 & 9058.2051 & 0.9783146 & -23.96206 & 2.75057 & -66.25000\end{array}$

$1513 \quad 6400.1460 \quad 6668.8052 \quad 1.0419770-15.24870-4.27001 \quad-66.25000$

$\begin{array}{llllllll}1526 & 5711.4658 & 5600.6108 & 0.9805908 & -28.26048 & -5.14798 & -66.25000\end{array}$

$\begin{array}{lllllll}1527 & 5161.3184 & 5081.4795 & 0.9845313 & -28.26047 & -0.65095 & -66.25000\end{array}$

$\begin{array}{lllllll}1542 & 3779.1233 & 3498.1377 & 0.9256479 & -19.58683 & -9.64501 & -66.25000\end{array}$

$15436404.4609 \quad 6014.4795 \quad 0.9391078-23.92366-9.64501-66.25000$

$\begin{array}{lllllll}1588 & 7970.8433 & 7070.3589 & 0.8870277 & -15.24827 & -2.47834 & -66.25000\end{array}$

$\begin{array}{llllllll}1589 & 2013.9762 & 2340.8745 & 1.1623149 & -15.24913 & -6.06167 & -66.25000\end{array}$ 


\section{SECTION B-B}

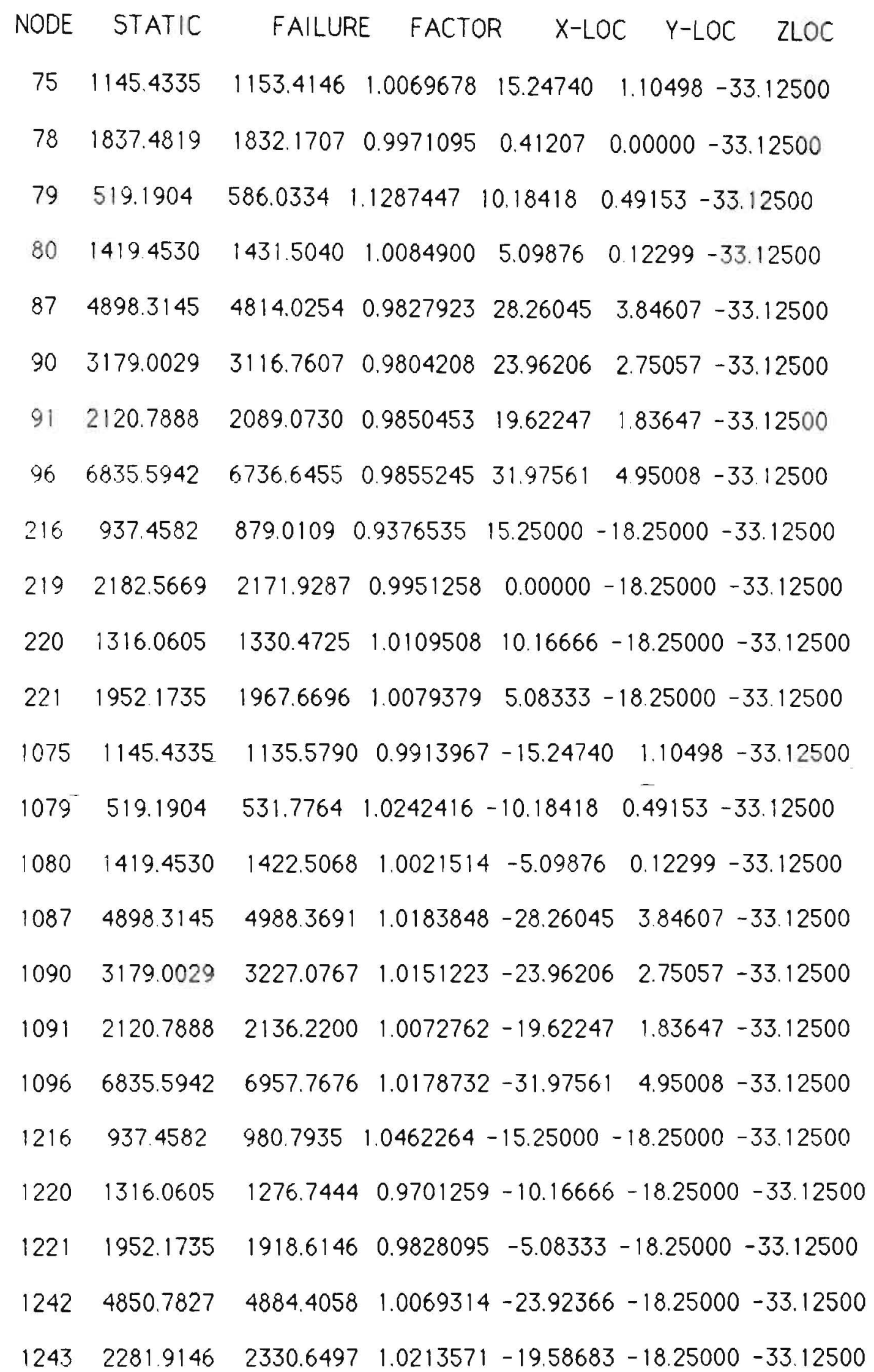




\section{STANCHION AND 3.5 in SUPPORT PLATES}

QUADRANT 1

NODE STATIC FAILURE FACTOR X-LOC Y-LOC ZLOC

$\begin{array}{lllllll}38 & 19647.1953 & 19646.3477 & 0.9999568 & 66.62949 & 23.63070 & -5.62500\end{array}$

$\begin{array}{lllllll}100 & 8247.0107 & 8260.5742 & 1.0016446 & 79.90000 & 23.63070 & 0.00000\end{array}$

$101 \quad 10987.7227 \quad 10362.1270 \quad 0.9976705 \quad 71.05299 \quad 23.63070 \quad 0.00000$

$\begin{array}{lllllll}102 & 9593.8721 & 9579.7275 & 0.9985257 & 75.47649 & 23.63070 & 0.00000\end{array}$

$103 \quad 5315.9082 \quad 5322.0034 \quad 1.0011466 \quad 79.90000 \quad 23.63070 \quad-11.25000$

$104 \quad 13225.1895 \quad 13230.2607 \quad 1.0003835 \quad 79.90000 \quad 23.63070 \quad-5.62500$

$105 \quad 6819.0015 \quad 6837.2109 \quad 1.0026704 \quad 75.47649 \quad 23.63070-11.25000$

$\begin{array}{lllllll}106 & 8967.4063 & 8962.4541 & 0.9994478 & 71.05299 & 23.63070 & -11.25000\end{array}$

$107 \quad 19577.0469 \quad 19575.2188 \quad 0.9999066 \quad 71.05299 \quad 23.63070 \quad-5.62500$

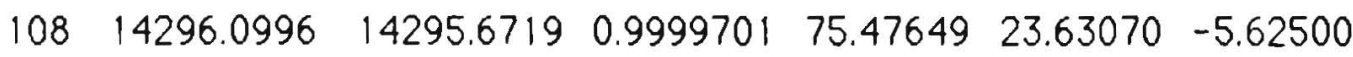

$\begin{array}{llllllll}111 & 8474.1807 & 8498.2451 & 1.0028397 & 15.25000 & -9.64501 & 0.00000\end{array}$

$124 \quad 11943.5117 \quad 11635.7227 \quad 0.97422296 \quad 28.26050 \quad-9.64501 \quad 0.00000$

$\begin{array}{lllllll}138 & 2833.5259 & 2814.6316 & \overline{0} .9933319 & 15.25000 & -9.64501 & 10.78100\end{array}$

$\begin{array}{lllllll}339 & 6096.6338 & 5993.0161 & 0.9830041 & 15.25000 & -9.64501 & 5.39050\end{array}$

$\begin{array}{lllllll}142 & 9376.6250 & 9238.4893 & 0.9852681 & 19.58683 & -9.64501 & 0.00000\end{array}$

$\begin{array}{llllllll}143 & 10627.6641 & 10376.8594 & 0.97640008 & 23.92366 & -9.64501 & 0.00000\end{array}$

$\begin{array}{llllllll}144 & 2313.8179 & 2186.9985 & 0.9451904 & 28.26050 & -9.64501 & 10.78100\end{array}$

$\begin{array}{lllllll}145 & 6125.6860 & 5905.7476 & 0.9640957 & 28.26050 & -9.64501 & 5.39050\end{array}$

$\begin{array}{llllllll}146 & 12254.0566 & 11960.7012 & 0.9760606 & 23.92366 & -9.64501 & 10.78100\end{array}$

$\begin{array}{llllllll}147 & 12552.8975 & 12247.3721 & 0.9756610 & 19.58683 & -9.64501 & 10.78100\end{array}$

$\begin{array}{lllllll}148 & 14107.5088 & 13791.8730 & 0.9776264 & 19.58683 & -9.64501 & 5.39050\end{array}$

$\begin{array}{lllllll}149 & 13893.0703 & 13524.7920 & 0.9734920 & 23.92366 & -9.64501 & 5.39050\end{array}$ 


\section{QUADRANT 2}

NODE StATIC FaILURE FACTOR X-LOC Y-LOC ZLOC

$\begin{array}{lllllll}438 & 19647.1953 & 19648.1934 & 1.0000508 & 66.62949 & 23.63070 & -60.62500\end{array}$

$\begin{array}{lllllll}500 & 8247.0107 & 8222.6953 & 0.9970516 & 79.90000 & 23.63070 & -66.25000\end{array}$

$\begin{array}{lllllll}501 & 10987.7227 & 11031.6240 & 1.0039955 & 71.05299 & 23.63070 & -66.25000\end{array}$

$\begin{array}{lllllll}502 & 9593.8721 & 9618.6914 & 1.0025870 & 75.47649 & 23.63070 & -66.25000\end{array}$

$\begin{array}{lllllll}503 & 5315.3082 & 5306.2256 & 0.9981785 & 79.90000 & 23.63070 & -55.00000\end{array}$

$\begin{array}{lllllll}504 & 13225.1895 & 13216.6895 & 0.9993573 & 79.90000 & 23.63070 & -60.62500\end{array}$

$\begin{array}{lllllll}505 & 6819.0015 & 6786.2065 & 0.9951907 & 75.47649 & 23.63070 & -55.00000\end{array}$

$\begin{array}{lllllll}506 & 8967.4063 & 8979.3896 & 1.0013363 & 71.05299 & 23.63070 & -55.00000\end{array}$

$\begin{array}{lllllll}507 & 19577.0469 & 19580.5547 & 1.0001792 & 71.05299 & 23.63070 & -60.62500\end{array}$

$\begin{array}{lllllll}508 & 14296.0996 & 14297.0342 & 1.0000653 & 75.47649 & 23.63070 & -60.62500\end{array}$

$\begin{array}{llllllll}511 & 8474.1807 & 6799.0254 & 0.8023224 & 15.25000 & -9.64501 & -66.25000\end{array}$

$\begin{array}{llllllll}524 & 11943.5117 & 12552.6133 & 1.0509986 & 28.26050 & -9.64501 & -66.25000\end{array}$

$\begin{array}{lllllll}538 & 2833.5259 & 3287.7349 & 1.1602981 & 15.25000 & -9.64501 & -77.03100\end{array}$

$\begin{array}{llllllll}539 & 6096.6338 & 4892.1211 & 0.8024299 & 15.25000 & -9.64501 & -71.64000\end{array}$

$\begin{array}{lllllll}542 & 9376.6250 & 8161.4751 & 0.8704064 & 19.58683 & -9.64501 & -66.25000\end{array}$

$\begin{array}{llllllll}543 & 10627.6641 & 11910.8281 & 1.1207381 & 23.92366 & -9.64501 & -66.25000\end{array}$

$\begin{array}{lllllll}544 & 2313.8179 & 2488.1580 & 1.0753474 & 28.26050 & -9.64501 & -77.03100\end{array}$

$\begin{array}{lllllll}545 & 6125.6860 & 7005.7715 & 1.1436713 & 28.26050 & -9.64501 & -71.64000\end{array}$

$\begin{array}{llllllll}546 & 12254.0566 & 16725.5313 & 1.3648975 & 23.92366 & -9.64501 & -77.03100\end{array}$

$\begin{array}{lllllll}547 & 12552.8975 & 7792.6753 & 0.6207870 & 19.58683 & -9.64501 & -77.03100\end{array}$

$\begin{array}{lllllll}548 & 14107.5088 & 9425.7988 & 0.6681406 & 19.58683 & -9.64501 & -71.64000\end{array}$

$\begin{array}{llllllll}549 & 13893.0703 & 19137.4336 & 1.3774805 & 23.92366 & -9.64501 & -71.64000\end{array}$ 


\section{QUADRANT 3}

NODE STATIC FAILURE FACTOR X-LOC Y-LOC ZLOC

$\begin{array}{lllllll}1038 & 19647.1953 & 19648.0586 & 1.0000440 & -66.62949 & 23.63070 & -5.62500\end{array}$

$\begin{array}{lllllll}1100 & 8247.0107 & 8235.2109 & 0.9985692 & -79.90000 & 23.63070 & 0.00000\end{array}$

$\begin{array}{lllllll}1101 & 10987.7227 & 11010.2012 & 1.0020458 & -71.05299 & 23.63070 & 0.00000\end{array}$

$\begin{array}{lllllll}1102 & 9593.8721 & 9606.2852 & 1.0012939 & -75.47649 & 23.63070 & 0.00000\end{array}$

$\begin{array}{lllllll}1103 & 5315.9082 & 5310.9199 & 0.9990616 & -79.90000 & 23.63070 & -11.25000\end{array}$

$\begin{array}{lllllll}1104 & 13225.1895 & 13220.9014 & 0.9996758 & -79.90000 & 23.63070 & -5.62500\end{array}$

$\begin{array}{lllllll}1105 & 6819.0015 & 6803.3247 & 0.9977010 & -75.47649 & 23.63070 & -11.25000\end{array}$

$\begin{array}{lllllll}1106 & 8967.4063 & 8971.6631 & 1.0004747 & -71.05299 & 23.63070 & -11.25000\end{array}$

$\begin{array}{lllllll}1107 & 19577.0469 & 19578.6660 & 1.0000827 & -71.05299 & 23.63070 & -5.62500\end{array}$

$\begin{array}{llllllll}1108 & 14296.0996 & 14296.4980 & 1.0000279 & -75.47649 & 23.63070 & -5.62500\end{array}$

$\begin{array}{llllllll}1111 & 8474.1807 & 8460.9297 & 0.9984363 & -15.25000 & -9.64501 & 0.00000\end{array}$

$\begin{array}{lllllll}1124 & 11943.5117 & 12233.1592 & 1.0242515 & -28.26050 & -9.64501 & 0.00000\end{array}$

$\begin{array}{lllllll}1138 & 2833.5259 & 2855.0281 & 1.0075885 & -15.25000 & -9.64501 & 10.78100\end{array}$

$\begin{array}{lllllll}11.39 & 6096.6338 & 6198.3765 & 1.0166883 & -15.25000 & -9.64501 & 5.39050\end{array}$

$1142 \quad 9376.6250 \quad 9513.9150 \quad 1.0146418-19.58683 \quad-9.64501 \quad 0.00000$

$\begin{array}{lllllll}1143 & 10627.6641 & 10869.9805 & 1.0228006 & -23.92366 & -9.64501 & 0.00000\end{array}$

$\begin{array}{lllllll}1144 & 2313.8179 & 2433.6897 & 1.0518069 & -28.26050 & -9.64501 & 10.78100\end{array}$

$1145 \quad 6125.6860 \quad 6336.8384 \quad 1.0344700 \quad-28.26050 \quad-9.64501 \quad 5.39050$

$\begin{array}{lllllll}1146 & 12254.0566 & 12547.9053 & 1.0239797 & -23.92366 & -9.64501 & 10.78100\end{array}$

$\begin{array}{lllllll}1147 & 12552.8975 & 12856.7500 & 1.0242058 & -19.58683 & -9.64501 & 10.78100\end{array}$

$\begin{array}{lllllll}1148 & 14107.5088 & 14423.3311 & 1.0223868 & -19.58683 & -9.64501 & 5.39050\end{array}$

$\begin{array}{lllllll}1149 & 13893.0703 & 14260.0693 & 1.0264159 & -23.92366 & -9.64501 & 5.39050\end{array}$ 
NODE STATIC FAILURE FACTOR X-LOC Y-LOC ZLOC $1438 \quad 19647.1953 \quad 19646.4590 \quad 0.9999625 \quad-66.62949 \quad 23.63070 \quad-60.62500$ $\begin{array}{lllllll}1500 & 8247.0107 & 8261.2793 & 1.0017302 & -79.90000 & 23.63070 & -66.25000\end{array}$ $\begin{array}{llllllll}1501 & 10987.7227 & 10961.1621 & 0.9975827 & -71.05299 & 23.63070 & -66.25000\end{array}$ $\begin{array}{lllllll}1502 & 9593.8721 & 9579.0615 & 0.9984562 & -75.47649 & 23.63070 & -66.25000\end{array}$

$\begin{array}{lllllll}1503 & 5315.9082 & 5322.2690 & 1.0011966 & -79.90000 & 23.63070 & -55.00000\end{array}$

$\begin{array}{lllllll}1504 & 13225.1895 & 13230.5107 & 1.0004023 & -79.90000 & 23.63070 & -60.62500\end{array}$

$\begin{array}{lllllll}1505 & 6819.0015 & 6838.2900 & 1.0028286 & -75.47649 & 23.63070 & -55.00000\end{array}$

$1506 \quad 8967.4063 \quad 8961.8096 \quad 0.9993759-71.05299 \quad 23.63070-55.00000$

$\begin{array}{lllllll}1507 & 19577.0469 & 19575.0195 & 0.9998965 & -71.05299 & 23.63070 & -60.62500\end{array}$

$\begin{array}{llllllll}1508 & 14296.0996 & 14295.5840 & 0.9999639 & -75.47649 & 23.63070 & -60.62500\end{array}$

$1511 \quad 8474.1807 \quad 8598.4824 \quad 1.0146683-15.25000 \quad-9.64501-66.25000$

$\begin{array}{lllllll}1524 & 11943.5117 & 11789.3838 & 0.9870952 & -28.26050 & -9.64501 & -66.25000\end{array}$

$\begin{array}{llllllll}1538 & 2833.5259 & 2868.1726 & 1.0122274 & -15.25000 & -9.64501 & -77.03100\end{array}$

$1539 \quad 6096.6338 \quad 6081.4976 \quad 0.9975173-15.25000 \quad-9.64501=-71.64000$

$\begin{array}{lllllll}1542 & 9376.6250 & 9459.0508 & 1.0087906 & -19.58683 & -9.64501 & -66.25000\end{array}$

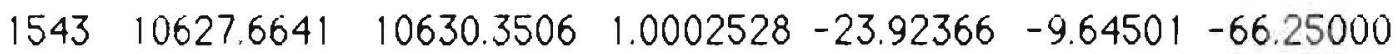

$1544 \quad 2313.8179 \quad 2248.8025 \quad 0.9719012 \quad-28.26050 \quad-9.64501-77.03100$

$1545 \quad 6125.6860 \quad 6076.3843 \quad 0.9919516-28.26050 \quad-9.64501-71.64000$

$\begin{array}{llllllll}1546 & 12254.0566 & 12564.6689 & 1.0253477 & -23.92366 & -9.64501 & -77.03100\end{array}$

$1547 \quad 12552.8975 \quad 12815.1484 \quad 1.0208917-19.58683 \quad-9.64501-77.03100$

$\begin{array}{llllllll}1548 & 14107.5088 & 14444.8857 & 1.0239147 & -19.58683 & -9.64501 & -71.64000\end{array}$

$\begin{array}{llllllll}1549 & 13893.0703 & 14216.0986 & 1.0232511 & -23.92366 & -9.64501 & -71.64000\end{array}$ 


\section{ARM CONNECT}

\section{QUADRANT 1}

NODE STATIC FAILURE FACTOR X-LOC Y-LOC ZLOC

$121 \quad 14662.2695 \quad 14924.6592 \quad 1.0178956 \quad 28.26050 \quad-9.64501-11.25000$

$\begin{array}{lllllll}124 & 9262.8857 & 9040.2852 & 0.9759685 & 28.26050 & -9.64501 & 0.00000\end{array}$

$125 \quad 5323.7173 \quad 5207.7720 \quad 0.9782210 \quad 28.26050 \quad-9.64501 \quad-5.62500$

$\begin{array}{lllllll}181 & 10540.7070 & 10354.2490 & 0.9823107 & 28.26050 & -18.25000 & 0.00000\end{array}$

$\begin{array}{lllllll}182 & 12551.1602 & 12267.7109 & 0.9774165 & 28.26050 & -13.94750 & 0.00000\end{array}$

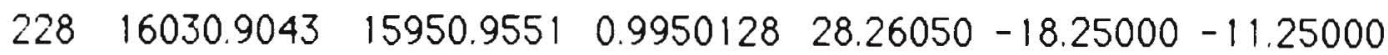

$231 \quad 8540.9580 \quad 8408.0000 \quad 0.9844329 \quad 28.26050-18.25000 \quad-5.62500$

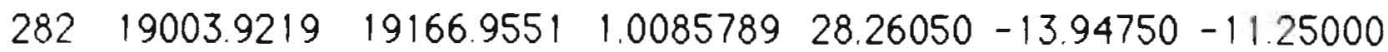

$\begin{array}{llllllll}305 & 6782.7661 & 6598.5850 & 0.9728457 & 28.26050 & -13.94750 & -5.62500\end{array}$

\section{QUADRANT 2}

NODE STATIC FAILURE FACTOR X-LOC Y-LOC ZLOC

$\begin{array}{lllllll}-521 & 14662.2695 & 14173.5430 & 0.9666677 & 28.26050 & -9.64501 & -55.00000\end{array}$

$\begin{array}{lllllll}524 & 9262.8857 & 9908.8857 & 1.0697407 & 28.26050 & -9.64501 & -66.25000\end{array}$

$\begin{array}{lllllll}525 & 5323.7173 & 5888.1465 & 1.1060216 & 28.26050 & -9.64501 & -60.62500\end{array}$

$\begin{array}{lllllll}581 & 10540.7070 & 10372.5850 & 0.9840502 & 28.26050 & -18.25000 & -66.25000\end{array}$

$\begin{array}{lllllll}582 & 12551.1602 & 12778.7686 & 1.0181345 & 28.26050 & -13.94750 & -66.25000\end{array}$

$\begin{array}{lllllll}628 & 16030.9043 & 16120.8691 & 1.0056120 & 28.26050 & -18.25000 & -55.00000\end{array}$

$\begin{array}{lllllll}631 & 8540.9580 & 8822.0215 & 1.0329077 & 28.26050 & -18.25000 & -60.62500\end{array}$

$\begin{array}{lllllll}682 & 19003.9219 & 18655.8008 & 0.9816816 & 28.26050 & -13.94750 & -55.00000\end{array}$

$\begin{array}{lllllll}705 & 6782.7661 & 7571.4819 & 1.1162823 & 28.26050 & -13.94750 & -60.62500\end{array}$ 


\section{QUADRANT 3}

NODE STATIC FAILURE FACTOR X-LOC Y-LOC ZLOC

$\begin{array}{lllllll}1121 & 14662.2695 & 14395.7334 & 0.9818217 & -28.26050 & -9.64501 & -11.25000\end{array}$

$\begin{array}{lllllll}1124 & 9262.8857 & 9473.3262 & 1.0227187 & -28.26050 & -9.64501 & 0.00000\end{array}$

$\begin{array}{lllllll}1125 & 5323.7173 & 5436.1372 & 1.0211169 & -28.26050 & -9.64501 & -5.62500\end{array}$

$\begin{array}{lllllll}1181 & 10540.7070 & 10721.9199 & 1.0171918 & -28.26050 & -18.25000 & 0.00000\end{array}$

$\begin{array}{lllllll}1182 & 12551.1602 & 12819.9844 & 1.0214183 & -28.26050 & -13.94750 & 0.00000\end{array}$

$1228 \quad 16030.9043 \quad 16080.5762 \quad 1.0030985-28.26050-18.25000-11.25000$

$1231 \quad 8540.9580 \quad 8664.6016 \quad 1.0144765-28.26050-18.25000 \quad-5.62500$

$1282 \quad 19003.9219 \quad 18823.5508 \quad 0.9905087-28.26050-13.94750-11.25000$

$1305 \quad 6782.7661 \quad 6962.0176 \quad 1.0264275 \quad-28.26050-13.94750 \quad-5.62500$

QUADRANT 4

NODE STATIC FAILURE FACTOR X-LOC Y-LOC ZLOC

$1521 \quad 14662.2695 \quad 14622.6006 \quad 0.9972945 \quad-28.26050 \quad-9.64501 \quad-55.00000$

$\begin{array}{lllllll}1524 & 9262.8857 & 9152.4102 & 0.9880733 & -28.26050 & -9.64501 & -66.25000\end{array}$

$1525 \quad 5323.7173 \quad 5279.7324 \quad 0.9917380-28.26050 \quad-9.64501 \quad-60.62500$

$1581 \quad 10540.7070 \quad 10416.3477 \quad 0.9882020-28.26050-18.25000 \quad-66.25000$

$\begin{array}{lllllll}1582 & 12551.1602 & 12374.0615 & 0.9858899 & -28.26050 & -13.94750 & -66.25000\end{array}$

$\begin{array}{lllllll}1628 & 16030.9043 & 15949.8291 & 0.9949425 & -28.26050 & -18.25000 & -55.00000\end{array}$

$1631 \quad 8540.9580 \quad 8445.2852 \quad 0.9887983-28.26050-18.25000-60.62500$

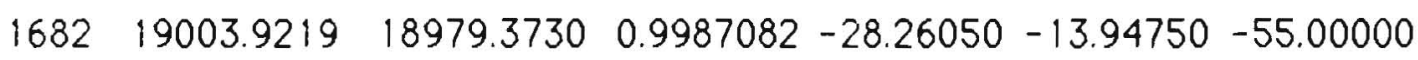

$\begin{array}{lllllll}1705 & 6782.7661 & 6685.4932 & 0.9856588 & -28.26050 & -13.94750 & -60.62500\end{array}$ 
NODE STATIC FAILURE FACTOR X-LOC Y-LOC ZLOC

$1 \quad 18590.9414 \quad 18594.4863 \quad 1.0001907 \quad 0.41207 \quad 0.00000 \quad 0.00000$

$\begin{array}{lllllll}7 & 13989.6982 & 13976.1563 & 0.9990320 & 0.41207 & 0.00000 & -11.25000\end{array}$

$\begin{array}{lllllll}10 & 17322.6250 & 17312.3027 & 0.9994041 & 0.41207 & 0.00000 & -5.62500\end{array}$

$\begin{array}{lllllll}51 & 6976.9146 & 6973.7173 & 0.9995418 & 0.41207 & 0.00000 & -19.12500\end{array}$

$\begin{array}{lllllll}54 & 10640.6738 & 10630.4678 & 0.9990408 & 0.41207 & 0.00000 & -15.18700\end{array}$

$\begin{array}{lllllll}78 & 1837.4819 & 1832.1707 & 0.9971095 & 0.41207 & 0.00000 & -33.12500\end{array}$

$\begin{array}{lllllll}81 & 1768.3112 & 1773.2323 & 1.0027829 & 0.41207 & 0.00000 & -28.45800\end{array}$

$\begin{array}{lllllll}82 & 3195.1631 & 3205.2837 & 1.0031675 & 0.41207 & 0.00000 & -23.79100\end{array}$

$\begin{array}{lllllll}201 & 15208.0459 & 15216.3975 & 1.0005492 & 0.00000 & -18.25000 & 0.00000\end{array}$

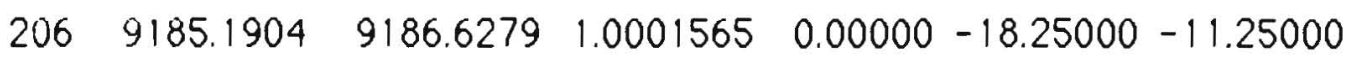

$\begin{array}{lllllll}209 & 12347.4111 & 12350.2119 & 1.0002269 & 0.00000 & -18.25000 & -5.62500\end{array}$

$\begin{array}{lllllll}212 & 6175.9893 & 6176.1074 & 1.0000191 & 0.00000 & -18.25000 & -16.62500\end{array}$

$\begin{array}{lllllll}222 & 2146.0637 & 2137.6670 & 0.9960874 & 0.00000 & -18.25000 & -27.62500\end{array}$

$\begin{array}{lllllll}223 & 3621.4751 & 3618.2639 & 0.9991133 & 0.00000 & -18.25000 & -22.12500\end{array}$

$\begin{array}{lllllll}401 & 18590.9414 & 18225.3848 & 0.9803368 & 0.41207 & 0.00000 & -66.25000\end{array}$

$\begin{array}{lllllll}407 & 13989.6982 & 13727.1631 & 0.9812337 & 0.41207 & 0.00000 & -55.00000\end{array}$

$\begin{array}{lllllll}410 & 17322.6250 & 16992.8535 & 0.9809629 & 0.41207 & 0.00000 & -60.62500\end{array}$

$\begin{array}{lllllll}451 & 6976.9146 & 6831.5249 & 0.9791613 & 0.41207 & 0.00000 & -47.12500\end{array}$

$\begin{array}{lllllll}454 & 10640.6738 & 10432.2891 & 0.9804162 & 0.41207 & 0.00000 & -51.06200\end{array}$

$\begin{array}{lllllll}481 & 1768.3112 & 1762.5574 & 0.9967462 & 0.41207 & 0.00000 & -37.79100\end{array}$

$\begin{array}{lllllll}482 & 3195.1631 & 3119.2341 & 0.9762363 & 0.41207 & 0.00000 & -42.45800\end{array}$

$\begin{array}{lllllll}601 & 15208.0459 & 14911.4434 & 0.9804970 & 0.00000 & -18.25000 & -66.25000\end{array}$

$\begin{array}{lllllll}606 & 9185.1904 & 9011.2051 & 0.9810581 & 0.00000 & -18.25000 & -55.00000\end{array}$

$\begin{array}{lllllll}609 & 12347.4111 & 12114.7822 & 0.9811597 & 0.00000 & -18.25000 & -60.62500\end{array}$

$\begin{array}{lllllll}612 & 6175.9893 & 6052.6807 & 0.9800342 & 0.00000 & -18.25000 & -49.62500\end{array}$

$\begin{array}{lllllll}622 & 2146.0637 & 2126.1782 & 0.9907340 & 0.00000 & -18.25000 & -38.62500\end{array}$

$\begin{array}{lllllll}623 & 3621.4751 & 3540.1851 & 0.9775533 & 0.00000 & -18.25000 & -44.12500\end{array}$ 


\section{APPENDDX 6 NUMBERFOUR}

Factor Listing 


\section{NUMBERFOUR WORST}

$\begin{array}{llllllll}\text { NODE } & \text { STATIC } & \text { FAILURE } & \text { FACTOR } & \text { X-LOC } & \text { Y-LOC } & \text { ZLOC } \\ 1119 & 6534.1294 & 4865.1260 & 0.7445714 & -15.25000 & -13.94750 & 0.00000 \\ 1134 & 539.0928 & 693.4313 & 1.2862930 & -19.58510 & 2.01868 & 10.78100 \\ 1139 & 5000.1997 & 3570.0325 & 0.7139780 & -15.25000 & -9.64501 & 5.39050 \\ 1141 & 5901.5444 & 4034.0669 & 0.6835612 & -15.24870 & -4.27001 & 5.39050 \\ 1145 & 4577.7803 & 5648.5156 & 1.2338984 & -28.26050 & -9.64501 & 5.39050 \\ 1153 & 6224.6641 & 7817.8853 & 1.2559530 & -28.26047 & -5.14798 & 5.39050 \\ 1195 & 4778.5171 & 3501.4636 & 0.7327511 & -19.59871 & -5.81784 & 0.00000 \\ 1519 & 6534.1294 & 4863.5645 & 0.7443324 & -15.25000 & -13.94750 & -66 & 25000 \\ 1534 & 539.0928 & 693.7038 & 1.2867984 & -19.58510 & 2.01868 & -77.03100 \\ 1539 & 5000.1997 & 3569.3191 & 0.7138353 & -15.25000 & -9.64501 & -71.64000 \\ 1541 & 5901.5444 & 4034.0757 & 0.6835627 & -15.24870 & -4.27001 & -71.64000 \\ 1545 & 4577.7803 & 5647.3994 & 1.2336545 & -28.26050 & -9.64501 & -71.64000 \\ 1553 & 6224.6641 & 7818.0493 & 1.2559793 & -28.26047 & -5.14798 & -71.64000 \\ 1595 & 4778.5171 & 3502.5527 & 0.7329790 & -19.59871 & -5.81784 & -66.25000\end{array}$




\section{SECTION A-A}

\section{QUADRANT 1}

NODE STATIC FAILURE FACTOR X-LOC Y-LOC ZLOC

$\begin{array}{llllllll}14 & 11704.3320 & 11326.7246 & 0.9677378 & 19.62247 & 1.83647 & 0.00000\end{array}$

$\begin{array}{lllllll}15 & 9258.9902 & 9211.0342 & 0.9948206 & 23.96206 & 2.75057 & 0.00000\end{array}$

$\begin{array}{lllllll}113 & 6400.1460 & 6757.6450 & 1.0558579 & 15.24870 & -4.27001 & 0.00000\end{array}$

$\begin{array}{lllllll}126 & 5711.4658 & 5754.7256 & 1.0075742 & 28.26048 & -5.14798 & 0.00000\end{array}$

$\begin{array}{lllllll}127 & 5161.3184 & 5203.0288 & 1.0080813 & 28.26047 & -0.65095 & 0.00000\end{array}$

$\begin{array}{llllllll}142 & 3779.1233 & 3523.8606 & 0.9324545 & 19.58683 & -9.64501 & 0.00000\end{array}$

$143 \quad 6404.4614 \quad 6141.8330 \quad 0.9589929 \quad 23.92366 \quad-9.64501 \quad 0.00000$

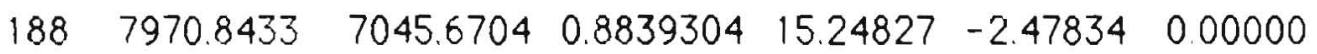

$\begin{array}{llllllll}189 & 2013.9762 & 2397.6921 & 1.1905266 & 15.24913 & -6.06167 & 0.00000\end{array}$

\section{QUADRANT 2}

NODE STATIC FAILURE FACTOR X-LOC Y-LOC ZLOC

$\begin{array}{llllllll}414 & 11704.3320 & -11325.3486 & 0.9676203 & 19.62247 & 1.83647 & -66.25000\end{array}$

$\begin{array}{lllllll}415 & 9258.9902 & 9210.4512 & 0.9947577 & 23.96206 & 2.75057 & -66.25000\end{array}$

$\begin{array}{lllllll}513 & 6400.1460 & 6759.2915 & 1.0561152 & 15.24870 & -4.27001 & -66.25000\end{array}$

$\begin{array}{lllllll}526 & 5711.4658 & 5753.4727 & 1.0073549 & 28.26048 & -5.14798 & -66.25000\end{array}$

$\begin{array}{lllllll}527 & 5161.3184 & 5202.4111 & 1.0079616 & 28.26047 & -0.65095 & -66.25000\end{array}$

$\begin{array}{lllllll}542 & 3779.1233 & 3522.7603 & 0.9321634 & 19.58683 & -9.64501 & -66.25000\end{array}$

$\begin{array}{lllllll}543 & 6404.4609 & 6139.2422 & 0.9585884 & 23.92366 & -9.64501 & -66.25000\end{array}$

$\begin{array}{lllllll}588 & 7970.8433 & 7042.1489 & 0.8834886 & 15.24827 & -2.47834 & -66.25000\end{array}$

$\begin{array}{lllllll}589 & 2013.9762 & 2399.3064 & 1.1913280 & 15.24913 & -6.06167 & -66.25000\end{array}$ 
NODE STATIC FAILURE FACTOR X-LOC Y-LOC ZLOC $\begin{array}{lllllll}1014 & 11704.3320 & 11297.2842 & 0.9652225 & -19.62247 & 1.83647 & 0.00000\end{array}$

$\begin{array}{lllllll}1015 & 9258.9902 & 9198.8076 & 0.9935001 & -23.96206 & 2.75057 & 0.00000\end{array}$

$\begin{array}{lllllll}1113 & 6400.1460 & 4905.0737 & 0.7664003 & -15.24870 & -4.27001 & 0.00000\end{array}$

$\begin{array}{lllllll}1126 & 5711.4658 & 5872.4146 & 1.0281799 & -28.26048 & -5.14798 & 0.00000\end{array}$

$\begin{array}{lllllll}1127 & 5161.3184 & 5359.8398 & 1.0384634 & -28.26047 & -0.65095 & 0.00000\end{array}$

$\begin{array}{lllllll}1142 & 3779.1233 & 2941.4873 & 0.7783518 & -19.58683 & -9.64501 & 0.00000\end{array}$

$\begin{array}{lllllll}1143 & 6404.4614 & 5448.1128 & 0.8506746 & -23.92366 & -9.64501 & 0.00000\end{array}$

$\begin{array}{llllllll}1188 & 7970.8433 & 6427.2773 & 0.8063485 & -15.24827 & -2.47834 & 0.00000\end{array}$

$\begin{array}{lllllll}1189 & 2013.9762 & 2095.5859 & 1.0405217 & -15.24913 & -6.06167 & 0.00000\end{array}$

\section{QUADRANT 4}

NODE STATIC FAILURE FACTOR X-LOC Y-LOC ZLOC

$\begin{array}{llllllll}1414 & 11704.3320 & 11299.7031 & 0.9654291 & -19.62247 & 1.83647 & -66.25000\end{array}$

- $\quad \begin{array}{lllllll}4415 & 9258.9902 & 9200.3994 & 0.9936720 & -23.96206 & 2.75057 & -66.25000\end{array}$

$1513 \quad 6400.1460 \quad 4905.1621 \quad 0.76641411-15.24870 \quad-4.27001-66.25000$

$\begin{array}{lllllll}1526 & 5711.4658 & 5872.8311 & 1.0282528 & -28.26048 & -5.14798 & -66.25000\end{array}$

$\begin{array}{llllllll}1527 & 5161.3184 & 5360.8711 & 1.0386631 & -28.26047 & -0.65095 & -66.25000\end{array}$

$1542 \quad 3779.1233 \quad 2941.4290 \quad 0.7783363-19.58683 \quad-9.64501 \quad-66.25000$

$1543 \cdot 6404.4609 \quad 5447.47850 .8505756-23.92366 \quad-9.64501-66.25000$

$\begin{array}{lllllll}1588 & 7970.8433 & 6427.5815 & 0.8063866 & -15.24827 & -2.47834 & -66.25000\end{array}$

$\begin{array}{llllllll}1589 & 2013.9762 & 2096.0745 & 1.0407642 & -15.24913 & -6.06167 & -66.25000\end{array}$ 


\section{SECTION B-B}

NOUE StATIt FAILURE FAtTOR $x$-LUt $y$-LOT ZLOE

$\begin{array}{lllllll}75 & 1145.4335 & 1110.6465 & 0.9696298 & 15.24740 & 1.10498 & -33.12500\end{array}$

$\begin{array}{lllllll}78 & 1837.4819 & 1813.6466 & 0.9870282 & 0.41207 & 0.00000 & -33.12500\end{array}$

$\begin{array}{lllllll}79 & 519.1904 & 477.3048 & 0.9193251 & 10.18418 & 0.49153 & -33.12500\end{array}$

$\begin{array}{lllllll}80 & 14194530 & 1401.3214 & 0.9872264 & 5.09876 & 0.12299 & -33.12500\end{array}$

$\begin{array}{lllllll}87 & 4898.3145 & 5073.9873 & 1.0358640 & 28.26045 & 3.84607 & -33.12500\end{array}$

$\begin{array}{lllllll}90 & 3179.0029 & 3270.9680 & 1.0289289 & 23.96206 & 2.75057 & -33.12500\end{array}$

$91 \quad 2120.7888 \quad 2143.7686 \quad 1.0108354 \quad 19.62247 \quad 1.83647 \quad-33.12500$

$\begin{array}{lllllll}96 & 6835.5942 & 7074.0869 & 1.0348898 & 31.97561 & 4.95008 & -33.12500\end{array}$

$\begin{array}{lllllll}216 & 937.4582 & 1009.5634 & 1.0769156 & 15.25000 & -18.25000 & -33.12500\end{array}$

$219 \quad 2182.5669 \quad 2154.99780 .9873685 \quad 0.00000-18.25000 \quad-33.12500$

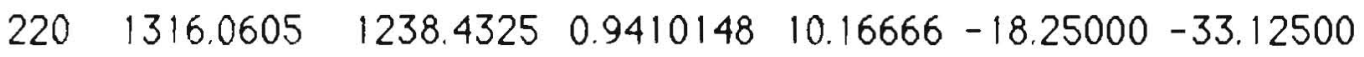

$221 \quad 1952.1735 \quad 1878.22010 .9621174 \quad 5.08333-18.25000-33.12500$

$\begin{array}{lllllll}1075 & 1145.4335 & 1141.9307 & 0.9969419 & -15.24740 & 1.10498 & -33.12500\end{array}$

$\begin{array}{lllllll}1079 & 519.1904 & 583.6582 & 1.1241698 & -10.18418 & 0.49153 & -33.12500\end{array}$

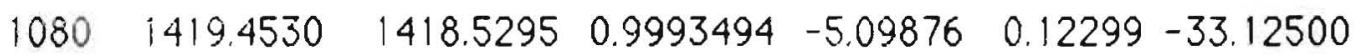

$\begin{array}{lllllll}1087 & 4898.3145 & 4732.7095 & 0.9661914 & -28.26045 & 3.84607 & -33.12500\end{array}$

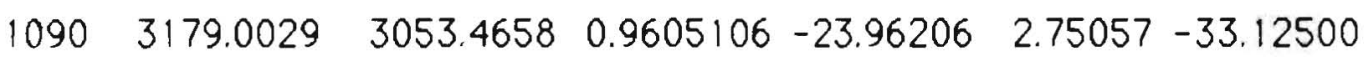

$\begin{array}{lllllll}1091 & 2120.7888 & 2046.8429 & 0.9651328 & -19.62247 & 1.83647 & -33.12500\end{array}$

$1096 \quad 6835.5942 \quad 6641.4946 \quad 0.9716046 \quad-31.97561 \quad 4.95008 \quad-33.12500$

$\begin{array}{lllllll}1216 & 937.4582 & 808.6712 & 0.8626211 & -15.25000 & -18.25000 & -33.12500\end{array}$

$1220 \quad 1316.0605 \quad 1342.1357 \quad 1.0198131-10.16666-18.25000-33.12500$

$1221 \quad 1952.1735 \quad 1973.8665 \quad 1.0111122-5.08333-18.25000-33.12500$

$1242 \quad 4850.7827 \quad 4727.5112 \quad 0.9745873-23.92366-18.25000-33.12500$

$1243 \quad 2281.9146 \quad 2139.8457 \quad 0.9377414-19.58683-18.25000-33.12500$ 


\section{STANCHION AND 3.5 in PLATE}

\begin{tabular}{ccccccccc}
\multicolumn{7}{c}{ QUADRANT 1} \\
NODE & STATIC & FAILURE & FACTOR & X-LOC & Y-LOC & ZLOC \\
38 & 19647.1953 & 19647.2617 & 1.0000033 & 66.62949 & 23.63070 & -5.62500 \\
100 & 8247.0107 & 8249.3184 & 1.0002798 & 79.90000 & 23.63070 & 0.00000 \\
101 & 10987.7227 & 10983.8721 & 0.9996496 & 71.05299 & 23.63070 & 0.00000 \\
102 & 9593.8721 & 9591.5986 & 0.9997630 & 75.47649 & 23.63070 & 0.00000 \\
103 & 5315.9082 & 5316.8491 & 1.0001770 & 79.90000 & 23.63070 & -11.25000 \\
104 & 13225.1895 & 13226.0088 & 1.0000620 & 79.90000 & 23.63070 & -5.62500 \\
105 & 6819.0015 & 6822.2559 & 1.0004772 & 75.47649 & 23.63070 & -11.25000 \\
106 & 8967.4063 & 8965.9883 & 0.9998419 & 71.05299 & 23.63070 & -11.25000 \\
107 & 19577.0469 & 19576.5957 & 0.9999769 & 71.05299 & 23.63070 & -5.52500 \\
108 & 14296.0996 & 14295.9385 & 0.9999887 & 75.47649 & 23.63070 & -5.62500 \\
111 & 8474.1807 & 8651.0000 & 1.0208657 & 15.25000 & -9.64501 & 0.00000 \\
124 & 11943.5117 & 12076.8330 & 1.0111626 & 28.26050 & -9.64501 & 0.00000 \\
138 & 2833.5259 & 2880.5356 & 1.0165906 & 15.25000 & -9.64501 & 10.78100 \\
139 & 6096.6338 & 6178.8779 & 1.0134901 & 15.25000 & -9.64501 & 5.39050 \\
142 & 9376.6250 & 9594.2002 & 1.0232040 & 19.58683 & -9.64501 & 0.00000 \\
143 & 10627.6641 & 10867.0879 & 1.0225284 & 23.92366 & -9.64501 & 0.00000 \\
144 & 2313.8179 & 2365.2698 & 1.0222368 & 28.26050 & -9.64501 & 10.78100 \\
145 & 6125.6860 & 6281.1919 & 1.0253859 & 28.26050 & -9.64501 & 5.39050 \\
146 & 12254.0566 & 12843.2969 & 1.04808553 & 23.92366 & -9.64501 & 10.78100 \\
147 & 12552.8975 & 13104.9707 & 1.0439798 & 19.58683 & -9.64501 & 10.78100 \\
148 & 14107.5088 & 14744.4043 & 1.0451459 & 19.58683 & -9.64501 & 5.39050
\end{tabular}




\section{QUADRANT 2}

NODE STATIC FAILURE FACTOR X-LOC Y-LOC ZLOC

$\begin{array}{lllllll}438 & 19647.1953 & 19647.2422 & 1.0000024 & 66.62949 & 23.63070 & -60.62500\end{array}$

$\begin{array}{lllllll}500 & 82470107 & 8249.4619 & 1.0002972 & 79.90000 & 23.63070 & -66.25000\end{array}$

$\begin{array}{lllllll}501 & 10987.7227 & 10983.5674 & 0.9996218 & 71.05299 & 23.63070 & -66.25000\end{array}$

$\begin{array}{lllllll}502 & 9593.8721 & 9591.4375 & 0.9997463 & 75.47649 & 23.63070 & -66.25000\end{array}$

$\begin{array}{lllllll}503 & 5315.9082 & 5316.9160 & 1.0001895 & 79.90000 & 23.63070 & -55.00000\end{array}$

$\begin{array}{lllllll}504 & 13225.1895 & 13226.0664 & 1.0000663 & 79.90000 & 23.63070 & -60.62500\end{array}$

$\begin{array}{lllllll}505 & 6819.0015 & 6822.4419 & 1.0005045 & 75.47649 & 23.63070 & -55.00000\end{array}$

$\begin{array}{lllllll}506 & 8967.4063 & 8965.9746 & 0.9998404 & 71.05299 & 23.63070 & -55.00000\end{array}$

$\begin{array}{lllllll}507 & 19577.0469 & 19576.5762 & 0.9999760 & 71.05299 & 23.63070 & -60.62500\end{array}$

$\begin{array}{lllllll}508 & 14296.0996 & 14295.9336 & 0.9999884 & 75.47649 & 23.63070 & -60.62500\end{array}$

$\begin{array}{lllllll}511 & 8474.1807 & 8652.5342 & 1.0210466 & 15.25000 & -9.64501 & -66.25000\end{array}$

$\begin{array}{lllllll}524 & 11943.5117 & 12074.7305 & 1.0109866 & 28.26050 & -9.6450 & -66.25000\end{array}$

$\begin{array}{llllllll}538 & 2833.5259 & 2880.9033 & 1.0167203 & 15.25000 & -9.64501 & -77.03100\end{array}$

$\begin{array}{lllllll}539 & 6096.6338 & 6178.7417 & 1.0134678 & 15.25000_{-}-9.64501 & -71.64000\end{array}$

$\begin{array}{llllllll}542 & 9376.6250 & 9594.5439 & 1.0232407 & 19.58683 & -9.64501 & -66.25000\end{array}$

$\begin{array}{llllllll}543 & 10627.6641 & 10866.2500 & 1.0224495 & 23.92366 & -9.64501 & -66.25000\end{array}$

$\begin{array}{llllllll}544 & 2313.8179 & 2364.3181 & 1.0218256 & 28.26050 & -9.64501 & -77.03100\end{array}$

$\begin{array}{lllllll}545 & 6125.6860 & 6279.9922 & 1.0251900 & 28.26050 & -9.64501 & -71.64000\end{array}$

$\begin{array}{llllllll}546 & 12254.0566 & 12844.1846 & 1.0481578 & 23.92366 & -9.64501 & -77.03100\end{array}$

$\begin{array}{lllllll}547 & 12552.8975 & 13105.5488 & 1.0440258 & 19.58683 & -9.6450 & -77.03100\end{array}$

$\begin{array}{llllllll}548 & 14107.5088 & 14745.4531 & 1.0452203 & 19.58683 & -9.64501 & -71.64000\end{array}$

$\begin{array}{llllllll}549 & 13893.0703 & 14566.6504 & 1.0484831 & 23.92366 & -9.64501 & -71.64000\end{array}$ 
QUADRANT 3

NODE STATIC FAILURE FACTOR X-LOC Y-LOC ZLOC

$\begin{array}{lllllll}1038 & 19647.1953 & 19647.2012 & 1.0000004 & -66.62949 & 23.63070 & -5.62500\end{array}$

$\begin{array}{lllllll}1100 & 8247.0107 & 8236.4814 & 0.9987233 & -79.90000 & 23.63070 & 0.00000\end{array}$

$\begin{array}{lllllll}1101 & 10987.7227 & 11005.5166 & 1.0016195 & -71.05299 & 23.63070 & 0.00000\end{array}$

$\begin{array}{lllllll}1102 & 9593.8721 & 9604.2412 & 1.0010808 & -75.47649 & 23.63070 & 0.00000\end{array}$

$\begin{array}{lllllll}1103 & 5315.9082 & 5311.6968 & 0.9992078 & -79.90000 & 23.63070 & -11.25000\end{array}$

$\begin{array}{lllllll}1104 & 13225.1895 & 13221.5352 & 0.9997237 & -79.90000 & 23.63070 & -5.62500\end{array}$

$\begin{array}{lllllll}1105 & 6819.0015 & 6804.4390 & 0.9978644 & -75.47649 & 23.63070 & -11.25000\end{array}$

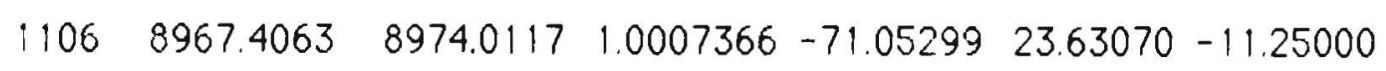

$\begin{array}{lllllll}1107 & 19577.0469 & 19578.5508 & 1.0000768 & -71.05299 & 23.63070 & -5.62500\end{array}$

$\begin{array}{lllllll}1108 & 14296.0996 & 14296.4922 & 1.0000274 & -75.47649 & 23.63070 & -5.62500\end{array}$

$\begin{array}{lllllll}1111 & 8474.1807 & 6850.4917 & 0.8083957 & -15.25000 & -9.64501 & 0.00000\end{array}$

$\begin{array}{lllllll}1124 & 11943.5117 & 12238.7881 & 1.0247227 & -28.26050 & -9.64501 & 0.00000\end{array}$

$\begin{array}{lllllll}1138 & 2833.5259 & 3346.2517 & 1.1809498 & -15.25000 & -9.64501 & 10.78100\end{array}$

$\begin{array}{lllllll}1139 & 6096.6338 & 4868.6694 & 0.7985832 & -15.25000 & -9.64501 & 5.39050\end{array}$

$\begin{array}{lllllll}1142 & 9376.6250 & 8080.1689 & 0.8617353 & -19.58683 & -9.64501 & 0.00000\end{array}$

$\begin{array}{llllllll}1143 & 10627.6641 & 11649.0137 & 1.0961030 & -23.92366 & -9.64501 & 0.00000\end{array}$

$\begin{array}{lllllll}1144 & 2313.8179 & 2450.9060 & 1.0592476 & -28.26050 & -9.64501 & 10.78100\end{array}$

$\begin{array}{lllllll}1145 & 6125.6860 & 6851.5029 & 1.1184875 & -28.26050 & -9.64501 & 5.39050\end{array}$

$\begin{array}{lllllll}1146 & 12254.0566 & 16329.2227 & 1.3325565 & -23.92366 & -9.64501 & 10.78100\end{array}$

$\begin{array}{lllllll}1147 & 12552.8975 & 7606.4595 & 0.6059525 & -19.58683 & -9.64501 & 10.78100\end{array}$

$\begin{array}{lllllll}1148 & 14107.5088 & 9233.9512 & 0.6545416 & -19.58683 & -9.64501 & 5.39050\end{array}$

$\begin{array}{lllllll}1149 & 13893.0703 & 18675.4629 & 1.3442286 & -23.92366 & -9.64501 & 5.39050\end{array}$

$\begin{array}{lllllll}1438 & 19647.1953 & 19647.1953 & 1.0000000 & -66.62949 & 23.63070 & -60.62500\end{array}$ 


\section{QUADRANT 4}

\begin{tabular}{|c|c|c|c|c|c|c|}
\hline NODE & STATIC & FAILURE & FACTOR & $X-L O C$ & $Y-L O C$ & ZLOC \\
\hline 500 & 8247.0107 & 8236.4561 & 0.9987202 & -79.90000 & $23.63070-$ & -66.25000 \\
\hline 50 & 0987.7227 & 05.5381 & .0016214 & -71.05299 & 23.63070 & -66.25000 \\
\hline 502 & 9593.8721 & 9604.2588 & .0010827 & -75.47649 & $23.63070-$ & -66.25000 \\
\hline 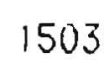 & 5315.9082 & 5311.6895 & 0.9992064 & -79.90000 & $23.63070-$ & -55.00000 \\
\hline 50 & 13225.1895 & 13221.5283 & 0.9997232 & $2-79.90000$ & 23.63070 & -60.6 \\
\hline 150 & 6819 & 6804.4019 & 0.9978590 & -75.47649 & $23.63070-$ & -55.00000 \\
\hline 50 & 8967 & 8974.0498 & 1.0007409 & -71.05299 & $23.63070-$ & -55 \\
\hline 0 & 577.0469 & 19578.5547 & 1.0000770 & -71.05299 & 23.63070 & -60.62500 \\
\hline 50 & 996 & 14296.4941 & 1.0000277 & $7-75.47649$ & 23.63070 & -60 \\
\hline 15 & 307 & 6849.3560 & 0.8082618 & -15.25000 & $-9.64501-$ & -66.25000 \\
\hline 52 & 5117 & 12236.6436 & 1.0245432 & -28.26050 & -9.645538 & $8-6$ \\
\hline 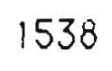 & 33.5259 & $3345.6338 \quad 1$ & $1.1807317-$ & -15.25000 & $-9.64501-$ & 77.0 \\
\hline 52 & 6096.6338 & 4867.5815 & 0.7984048 & -15.25000 & $-9.64501=$ & 000 \\
\hline דרו & 9376.6250 & $\overline{80} 78.8242$ & 0.8615919 & -19.5868 & -9.64501 & 5000 \\
\hline 154 & 10627.6641 & 11647.6816 & 1.095977 & -23.9236 & -9.64501 & -66.25000 \\
\hline 1544 & 2312.8179 & 2450.3433 & 1.059 & -28.2605 & -9.64501 & -77.031 \\
\hline 1545 & 6125.6860 & 6851.7090 & 1.1185211 & -28.26050 & -9.64501 & -71.64000 \\
\hline 1546 & 12254.0566 & 16331.8252 & 1.33276 & -23.9236 & -9.64501 & -77.031 \\
\hline 154 & 12552.8975 & 7607.6499 & 0.6060473 & $3-19.58683$ & $3-9.64501$ & -77.031 \\
\hline & 14107.5088 & 9234.04 & 0.654547 & -19.58683 & $3-9.64501$ & -71.64000 \\
\hline & & 60 & 1.3 & $2-23$ & & \\
\hline
\end{tabular}




\section{ARM CONNECT}

\section{OUADRANT 1}

NODE STATIC FAILURE FACTOR X-LOC Y-LOC ZLOC

$121 \quad 14662.2695 \quad 14361.5508 \quad 0.9794903 \quad 28.26050-9.64501-11.25000$

$\begin{array}{llllllll}124 & 9262.8857 & 9361.3965 & 1.0106350 & 28.26050 & -9.64501 & 0.00000\end{array}$

$125 \quad 5323.7173 \quad 5389.6025 \quad 1.0123758 \quad 28.26050-9.64501 \quad-5.62500$

$\begin{array}{lllllll}181 & 10540.7070 & 10597.0186 & 1.0053422 & 28.26050 & -18.25000 & 0.00000\end{array}$

$\begin{array}{lllllll}182 & 12551.1602 & 12641.7402 & 1.0072168 & 28.26050 & -13.94750 & 0.00000\end{array}$

$228 \quad 16030.9043 \quad 15999.17190 .9980205 \quad 28.26050-18.25000-11.25000$

$231 \quad 8540.9580 \quad 8568.4609 \quad 1.0032201 \quad 28.26050-18.25000 \quad-5.62500$

$\begin{array}{lllllll}282 & 19003.9219 & 18802.1660 & 0.9893835 & 28.26050 & -13.94750 & -11.25000\end{array}$

$\begin{array}{lllllll}305 & 6782.7661 & 6862.4082 & 1.0117419 & 28.26050 & -13.94750 & -5.62500\end{array}$

\section{QUADRANT 2}

NODE STATIC FAILURE FACTOR X-LOC Y-LOC ZLOC

$\begin{array}{llllllll}\text { - } & 521 & 14662.2695 & 14363.4014 & 0.9796165 & 28.26050 & -9.64501 & -55.00000\end{array}$

$\begin{array}{lllllll}524 & 9262.8857 & 9359.7129 & 1.0104532 & 28.26050 & -9.64501 & -66.25000\end{array}$

$\begin{array}{lllllll}525 & 5323.7173 & 5389.1201 & 1.0122852 & 28.26050 & -9.64501 & -60.62500\end{array}$

$\begin{array}{lllllll}581 & 10540.7070 & 10594.7178 & 1.0051240 & 28.26050 & -18.25000 & -66.25000\end{array}$

$\begin{array}{lllllll}582 & 12551.1602 & 12639.0977 & 1.0070063 & 28.26050 & -13.94750 & -66.25000\end{array}$

$\begin{array}{lllllll}628 & 16030.9043 & 15999.7861 & 0.9980589 & 28.26050 & -18.25000 & -55.00000\end{array}$

$\begin{array}{lllllll}631 & 8540.9580 & 8567.5049 & 1.0031081 & 28.26050 & -18.25000 & -60.62500\end{array}$

$\begin{array}{lllllll}682 & 19003.9219 & 18804.3223 & 0.9894969 & 28.26050 & -13.94750 & -55.00000\end{array}$

$\begin{array}{lllllll}705 & 6782.7661 & 6861.3423 & 1.0115846 & 28.26050 & -13.94750 & -60.62500\end{array}$ 
QUADRANT 3

NODE STATIC FAILURE FACTOR X-LOC Y-LOC ZLOC

$\begin{array}{lllllll}1121 & 14662.2695 & 14435.0400 & 0.9845024 & -28.26050 & -9.64501 & -11.25000\end{array}$

$\begin{array}{lllllll}1124 & 9262.8857 & 9678.4131 & 1.0448594 & -28.26050 & -9.64501 & 0.00000\end{array}$

$\begin{array}{llllllll}1125 & 5323.7173 & 5751.7881 & 1.0804082 & -28.26050 & -9.64501 & -5.62500\end{array}$

$\begin{array}{lllllll}1181 & 10540.7070 & 10196.4961 & 0.9673446 & -28.26050 & -18.25000 & 0.00000\end{array}$

$\begin{array}{lllllll}1182 & 12551.1602 & 12499.0732 & 0.9958500 & -28.26050 & -13.94750 & 0.00000\end{array}$

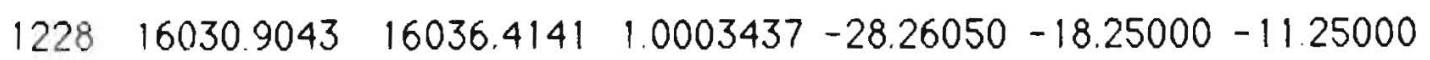

$1231 \quad 8540.9580 \quad 8686.1484 \quad 1.0169994-28.26050-18.25000 \quad-5.62500$

$\begin{array}{lllllll}1282 & 19003.9219 & 18808.8516 & 0.9897352 & -28.26050 & -13.94750 & -11.25000\end{array}$

$\begin{array}{lllllll}1305 & 6782.7661 & 7361.3481 & 1.0853018 & -28.26050 & -13.94750 & -5.62500\end{array}$

\section{QUADRANT 4}

NODE STATIC FAILURE FACTOR X-LOC Y-LOC ZLOC

$\begin{array}{lllllll}1521 & 14662.2695 & 14436.3906 & 0.9845945 & -28.26050 & -9.64501 & -55.00000\end{array}$

$\begin{array}{lllllll}1524 & 9262.8857 & 9676.4414 & 1.0446465 & -28.26050 & -9.64501 & -66.25000\end{array}$

$\begin{array}{lllllll}1525 & 5323.7173 & 5749.9414 & 1.0800613 & -28.26050 & -9.64501 & -60.62500\end{array}$

$\begin{array}{lllllll}1581 & 10540.7070 & 10195.9131 & 0.9672893 & -28.26050 & -18.25000 & -66.25000\end{array}$

$\begin{array}{lllllll}1582 & 12551.1602 & 12497.3945 & 0.9957163 & -28.26050 & -13.94750 & -66.25000\end{array}$

$\begin{array}{lllllll}1628 & 16030.9043 & 16036.5898 & 1.0003546 & -28.26050 & -18.25000 & -55.00000\end{array}$

$\begin{array}{lllllll}1631 & 8540.9580 & 8685.2510 & 1.0168942 & -28.26050 & -18.25000 & -60.62500\end{array}$

$\begin{array}{lllllll}1682 & 19003.9219 & 18810.0449 & 0.9897981 & -28.26050 & -13.94750 & -55.00000\end{array}$

$\begin{array}{lllllll}1705 & 6782.7661 & 7359.1597 & 1.0849792 & -28.26050 & -13.94750 & -60.62500\end{array}$ 


\section{TIE PLAIE}

NODE STATIC FAILURE FACTOR X-LOC Y-LOC ZLOC

$\begin{array}{llllllll}1 & 18590.9414 & 18231.5313 & 0.9806675 & 0.41207 & 0.00000 & 0.00000\end{array}$

$\begin{array}{lllllll}7 & 13989.6982 & 13720.7881 & 0.9807780 & 0.41207 & 0.00000 & -11.25000\end{array}$

$\begin{array}{lllllll}10 & 17322.6250 & 16990.4883 & 0.9808264 & 0.41207 & 0.00000 & -5.62500\end{array}$

$51 \quad 6976.9146 \quad 6834.0474 \quad 0.9795229 \quad 0.41207 \quad 0.00000-19.12500$

$\begin{array}{lllllll}54 & 10640.6738 & 10429.1543 & 0.9801216 & 0.41207 & 0.00000 & -15.18700\end{array}$

$\begin{array}{lllllll}78 & 1837.4819 & 1813.6466 & 0.9870282 & 0.41207 & 0.00000 & -33.12500\end{array}$

$\begin{array}{llllllll}81 & 1768.3112 & 1748.2881 & 0.9886767 & 0.41207 & 0.00000 & -28.45800\end{array}$

$\begin{array}{lllllll}201 & 15208.0459 & 14927.3467 & 0.9815427 & 0.00000 & -18.25000 & 0.00000\end{array}$

$\begin{array}{lllllll}206 & 9185.1904 & 9016.8994 & 0.9816780 & 0.00000 & -18.25000 & -11.25000\end{array}$

$\begin{array}{lllllll}209 & 12347.4111 & 12123.4717 & 0.9818634 & 0.00000 & -18.25000 & -5.62500\end{array}$

$\begin{array}{lllllll}212 & 6175.9893 & 6055.8711 & 0.9805508 & 0.00000 & -18.25000 & -16.62500\end{array}$

$\begin{array}{lllllll}219 & 2182.5669 & 2154.9978 & 0.9873685 & 0.00000 & -18.25000 & -33.12500\end{array}$

$\begin{array}{lllllll}222 & 2146.0637 & 2108.7981 & 0.9826354 & 0.00000 & -18.25000 & -27.62500\end{array}$

$\begin{array}{lllllll}223 & 3621.4751 & 3538.4832 & 0.9770834 & 0.00000 & -18.25000 & -22.12500\end{array}$

$\begin{array}{lllllll}401 & 18590.9414 & 18238.8047 & 0.9810587 & 0.41207 & 0.00000 & -66.25000\end{array}$

$\begin{array}{lllllll}407 & 13989.6982 & 13719.6523 & 0.9806968 & 0.41207 & 0.00000 & -55.00000\end{array}$

$\begin{array}{lllllll}410 & 17322.6250 & 16990.5781 & 0.9808316 & 0.41207 & 0.00000 & -60.62500\end{array}$

$\begin{array}{llllllll}451 & 6976.9146 & 6832.4688 & 0.9792966 & 0.41207 & 0.00000 & -47.12500\end{array}$

$\begin{array}{lllllll}454 & 10640.6738 & 10427.5078 & 0.9799669 & 0.41207 & 0.00000 & -51.06200\end{array}$

$\begin{array}{lllllll}481 & 1768.3112 & 1748.5225 & 0.9888093 & 0.41207 & 0.00000 & -37.79100\end{array}$

$\begin{array}{lllllll}482 & 3195.1631 & 3127.6179 & 0.9788602 & 0.41207 & 0.00000 & -42.45800\end{array}$

$\begin{array}{lllllll}601 & 15208.0459 & 14926.4502 & 0.9814838 & 0.00000 & -18.25000 & -66.25000\end{array}$

$\begin{array}{lllllll}606 & 9185.1904 & 9016.5889 & 0.9816442 & 0.00000 & -18.25000 & -55.00000\end{array}$

$\begin{array}{lllllll}609 & 12347.4111 & 12122.7559 & 0.9818055 & 0.00000 & -18.25000 & -60.62500\end{array}$

$\begin{array}{lllllll}612 & 6175.9893 & 6055.8369 & 0.9805452 & 0.00000 & -18.25000 & -49.62500\end{array}$

$\begin{array}{lllllll}622 & 2146.0637 & 2108.7649 & 0.9826199 & 0.00000 & -18.25000 & -38.62500\end{array}$

$\begin{array}{lllllll}623 & 3621.4751 & 3538.5681 & 0.9771069 & 0.00000 & -18.25000 & -44.12500\end{array}$ 


\section{APPENDIX 7 NUMBERFIVE}

Factor Listing 


\section{NUMBERFIVE WORST}

NODE STATIC FAILURE FACTOR X-LOC Y-LOC ZLOC

$\begin{array}{lllllll}63 & 5058.0073 & 6255.3447 & 1.2367212 & 40.91170 & 8.23438 & -19.12500\end{array}$

$\begin{array}{lllllll}75 & 1214.2780 & 5037.9116 & 4.1488948 & 15.24740 & 1.10498 & -33.12500\end{array}$

$\begin{array}{llllllll}77 & 1121.9081 & 4337.3657 & 3.8660617 & 15.24740 & 1.10498 & -28.45800\end{array}$

$\begin{array}{lllllll}83 & 4028.0276 & 8129.6084 & 2.0182602 & 5.09876 & 0.12299 & -23.79100\end{array}$

$\begin{array}{lllllll}84 & 3228.4978 & 6326.5479 & 1.9595950 & 10.18418 & 0.49153 & -23.79100\end{array}$

$\begin{array}{lllllll}86 & 1924.1449 & 6990.4146 & 3.6329980 & 10.18418 & 0.49153 & -28.45800\end{array}$

$88 \quad 5962.2173 \quad 7184.9082 \quad 1.2050732 \quad 28.26045 \quad 3.84607 \quad-23.79100$

$90 \quad 3170.8860 \quad 43 \$ 4.3813 \quad 1.3606232 \quad 23.96206 \quad 2.75057 \quad-33.12500$

$91 \quad 2845.5210 \quad 5009.4805 \quad 1.7604792 \quad 19.62247 \quad 1.83647 \quad-33.12500$

$\begin{array}{llllllll}92 & 4733.1221 & 5811.0273 & 1.2277366 & 19.62247 & 1.83647 & -23.79100\end{array}$

$93 \quad 5175.9380 \quad 6746.4570 \quad 1.3034270 \quad 23.96206 \quad 2.75057 \quad-23.79100$

$94 \quad 2421.5706 \quad 3969.0112 \quad 1.6390235 \quad 19.62247 \quad 1.83647 \quad-28.45800$

$95 \quad 3137.9849 \quad 4572.3472 \quad 1.4570966 \quad 23.96206 \quad 2.75057 \quad-28.45800$

$111 \quad 6053.9985 \quad 7963.8711 \quad 1.3154730 \quad 15.25000-9.64501 \quad 0.00000$

$\begin{array}{lllllll}112 & 5787.0298 & 7733.1177 & 1.3362844 & 15.25000 & -9.64501 & -5.62500\end{array}$

$\begin{array}{llllllll}113 & 6672.7446 & 8348.7227 & 1.2511678 & 15.24870 & -4.27001 & 0.00000\end{array}$

$114 \quad 2493.1843 \quad 4040.9524 \quad 1.6207997 \quad 15.24870 \quad-4.27001 \quad-5.62500$

$120 \quad 6524.2920 \quad 9393.4014 \quad 1.4397579 \quad 15.25000 \quad-13.94750 \quad-5.62500$

$\begin{array}{lllllll}133 & 618.8265 & 460.7569 & 0.7445656 & 23.92280 & 2.93238 & 10.78100\end{array}$

$\begin{array}{lllllll}139 & 5438.0469 & 6527.6367 & 1.2003642 & 15.25000 & -9.64501 & 5.39050\end{array}$

$\begin{array}{lllllll}141 & 6579.0015 & 7958.2290 & 1.2096409 & 15.24870 & -4.27001 & 5.39050\end{array}$

$\begin{array}{llllllll}142 & 3789.2974 & 2753.1521 & 0.7265601 & 19.58683 & -9.64501 & 0.00000\end{array}$

$\begin{array}{lllllll}146 & 720.4385 & 884.5084 & 1.2277361 & 23.92366 & -9.64501 & 10.78100\end{array}$

$\begin{array}{lllllll}147 & 895.4142 & 1125.6899 & 1.2571723 & 19.58683 & -9.64501 & 10.78100\end{array}$

$\begin{array}{lllllll}148 & 526.9106 & 669.5283 & 1.2706676 & 19.58683 & -9.64501 & 5.39050\end{array}$

$\begin{array}{lllllll}188 & 9572.9775 & 6707.5996 & 0.7006806 & 15.24827 & -2.47834 & 0.00000\end{array}$ 
NODE STATIC FAILURE FACTOR X-LOC Y-LOC ZLOC

$\begin{array}{llllllll}189 & 1850.8263 & 2622.8689 & 1.4171340 & 15.24913 & -606167 & 0.00000\end{array}$

$\begin{array}{lllllll}194 & 6172.1460 & 4312.0439 & 0.6986296 & 23.93646 & -5.51314 & 0.00000\end{array}$

$\begin{array}{llllllll}195 & 4537.6011 & 3193.2126 & 0.7037227 & 19.59871 & -5.81784 & 0.00000\end{array}$

$199 \quad 9574.3340 \quad 6191.4819 \quad 0.6466749 \quad 19.58683-13.94750 \quad 0.00000$

$\begin{array}{llllllll}476 & 4482.5850 & 7391.4624 & 1.6489285 & 15.24740 & 1.10498 & -42.45800\end{array}$

$\begin{array}{llllllll}477 & 4067.4087 & 6586.9727 & 1.6194519 & 15.24740 & 1.10498 & -37.79100\end{array}$

$\begin{array}{llllllll}492 & 2937.9636 & 4075.9580 & 1.3873413 & 19.62247 & 1.83647 & -42.45800\end{array}$

$\begin{array}{llllllll}494 & 3148.3630 & 5277.8384 & 1.6763754 & 19.62247 & 1.83647 & -37.79100\end{array}$

$\begin{array}{llllllll}495 & 3038.2180 & 3981.0769 & 1.3103329 & 23.96206 & 2.7505 ? & -37.79100\end{array}$

$497 \quad 5092.8276 \quad 6298.2798 \quad 1.2366960 \quad 33.11902 \quad 5.27150 \quad-38.99900$

$\begin{array}{lllllll}512 & 5603.2339 & 4056.3938 & 0.7239380 & 15.25000 & -9.64501 & -60.62500\end{array}$

$\begin{array}{lllllll}520 & 6347.5664 & 4345.3447 & 0.6845686 & 15.25000 & -13.94750 & -60.62500\end{array}$

$\begin{array}{lllllll}533 & 738.6489 & 1172.9719 & 1.5879966 & 23.92280 & 2.93238 & -77.03100\end{array}$

$\begin{array}{llllllll}534 & 770.9153 & 1334.7360 & 1.7313652 & 19.58510 & 2.01868 & -77.03100\end{array}$

$\begin{array}{lllllll}542 & 4080.1113 & 5856.6660 & 1.4354182 & 19.58683 & -9.64501 & -66.25000\end{array}$

$\begin{array}{lllllll}588 & 10556.6924 & 13547.4023 & 1.2832999 & 15.24827 & -2.47834 & -66.25000\end{array}$

$\begin{array}{lllllll}589 & 2271.6011 & 4967.1338 & 2.1866224 & 15.24913 & -6.06167 & -66.25000\end{array}$

$\begin{array}{lllllll}593 & 10118.9873 & 12564.6240 & 1.2416879 & 19.61059 & -1.99068 & -66.25000\end{array}$

$\begin{array}{lllllll}594 & 6707.6372 & 8549.3389 & 1.2745678 & 23.93646 & -5.51314 & -66.25000\end{array}$

$\begin{array}{llllllll}595 & 4904.3843 & 6732.2134 & 1.3726928 & 19.59871 & -5.81784 & -66.25000\end{array}$

$\begin{array}{lllllll}599 & 10249.5762 & 14247.6973 & 1.3900768 & 19.58683 & -13.94750 & -66.25000\end{array}$

$\begin{array}{lllllll}626 & 2175.5720 & 2734.0874 & 1.2567211 & 5.08333 & -18.25000 & -38.62500\end{array}$

$\begin{array}{lllllll}627 & 1817.0812 & 2281.1533 & 1.2553943 & 10.16666 & -18.25000 & -38.62500\end{array}$

$1075 \quad 1214.2780 \quad 4503.7134 \quad 3.7089641-15.24740 \quad 1.10498 \quad-33.12500$

$1077 \quad 1121.9081 \quad 3326.1904 \quad 2.9647620-15.24740 \quad 1.10498-28.45800$

$1086 \quad 1924.1449 \quad 4209.1440 \quad 2.1875401-10.18418 \quad 0.49153-28.45800$

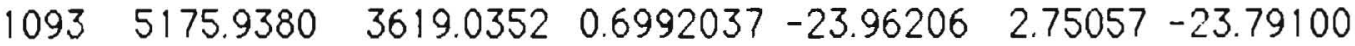


NODE STATIC FAILURE FACTOR X-LOC Y-LOC ZLOC

$\begin{array}{lllllll}1095 & 3137.9849 & 2262.1355 & 0.7208880 & -23.96206 & 2.75057 & -28.45800\end{array}$

$\begin{array}{lllllll}1112 & 5787.0298 & 4103.7036 & 0.7091209 & -15.25000 & -9.64501 & -5.62500\end{array}$

$\begin{array}{llllllll}1114 & 2493.1843 & 1827.6001 & 0.7330385 & -15.24870 & -4.27001 & -5.62500\end{array}$

$\begin{array}{lllllll}1120 & 6524.2920 & 4288.3623 & 0.6572916 & -15.25000 & -13.94750 & -5.62500\end{array}$

$\begin{array}{lllllll}1133 & 618.8265 & 1020.4310 & 1.6489776 & -23.92280 & 2.93238 & 10.78100\end{array}$

$\begin{array}{lllllll}1134 & 603.2517 & 1070.6567 & 1.7748092 & -19.58510 & 2.01868 & 10.78100\end{array}$

$\begin{array}{lllllll}1142 & 3789.2974 & 5674.7534 & 1.4975741 & -19.58683 & -9.64501 & 0.00000\end{array}$

$\begin{array}{lllllll}1143 & 6419.9321 & 7842.2515 & 1.2215474 & -23.92366 & -9.64501 & 0.00000\end{array}$

$\begin{array}{lllllll}1147 & 895.4142 & 665.9202 & 0.7437007 & -19.58683 & -9.64501 & 10.78100\end{array}$

$\begin{array}{lllllll}1148 & 526.9106 & 392.8171 & 0.7455099 & -19.58683 & -9.64501 & 5.39050\end{array}$

$\begin{array}{lllllll}1188 & 9572.9775 & 12650.5313 & 1.3214835 & -15.24827 & -2.47834 & 0.00000\end{array}$

$\begin{array}{lllllll}1189 & 1850.8263 & 4745.8682 & 2.5641890 & -15.24913 & -6.06167 & 0.00000\end{array}$

$\begin{array}{lllllll}1192 & 7223.4541 & 8672.1611 & 1.2005560 & -23.94926 & -1.38128 & 0.00000\end{array}$

$\begin{array}{lllllll}1193 & 9302.0889 & 11862.1553 & 1.2752142 & -19.61059 & -1.99068 & 0.00000\end{array}$

$\begin{array}{lllllll}1194 & 6172.1460 & 8205.7822 & 1.3294861 & -23.93646 & -5.51314 & 0.00000\end{array}$

$\begin{array}{llllllll}1195 & 4537.6011 & 6496.9121 & 1.4317945 & -19.59871 & -5.81784 & 0.00000\end{array}$

$\begin{array}{lllllll}1199 & 9574.3340 & 13796.6572 & 1.4410044 & -19.58683 & -13.94750 & 0.00000\end{array}$

$\begin{array}{lllllll}1216 & 913.7531 & 1119.7089 & 1.2253954 & -15.25000 & -18.25000 & -33.12500\end{array}$

$\begin{array}{lllllll}1226 & 2185.2837 & 2761.0942 & 1.2634946 & -5.08333 & -18.25000 & -27.62500\end{array}$

$1227 \quad 1874.0566 \quad 2355.3962 \quad 1.2568437-10.16666-18.25000 \quad-27.62500$

$\begin{array}{lllllll}1452 & 7318.2026 & 9372.7119 & 1.2807395 & -10.18418 & 0.49153 & -47.12500\end{array}$

$\begin{array}{lllllll}1459 & 7887.6396 & 9713.1953 & 1.2314451 & -23.96206 & 2.75057 & -47.12500\end{array}$

$\begin{array}{lllllll}1460 & 7685.8706 & 9693.2275 & 1.2611749 & -19.62247 & 1.83647 & -47.12500\end{array}$

$\begin{array}{lllllll}1463 & 4804.3564 & 6091.1470 & 1.2678382 & -40.91170 & 8.23438 & -47.125000\end{array}$

$\begin{array}{lllllll}1512 & 5603.2339 & 7410.7129 & 1.3225778 & -15.25000 & -9.64501 & -60.62500\end{array}$

$\begin{array}{llllllll}1513 & 6620.7393 & 8125.8618 & 1.2273345 & -15.24870 & -4.27001 & -66.25000\end{array}$

$\begin{array}{llllllll}1514 & 2737.3845 & 4113.3452 & 1.5026553 & -15.24870 & -4.27001 & -60.62500\end{array}$ 
NODE STATIC FAILURE FACTOR X-LOC Y-LOC ZLOC

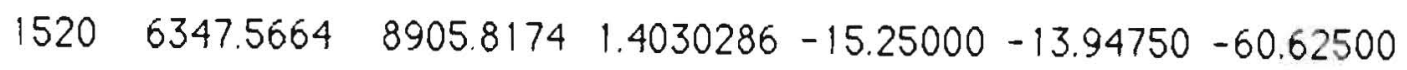

$1528 \quad 3458.7295 \quad 4215.4878 \quad 1.2187966-28.26048-0.65095 \quad-60.62500$

$\begin{array}{lllllll}1533 & 738.6489 & 495.6037 & 0.6709598 & -23.92280 & 2.93238 & -77.03100\end{array}$

$\begin{array}{lllllll}1534 & 770.9153 & 547.1519 & 0.7097431 & -19.58510 & 2.01868 & -77.03100\end{array}$

$1541 \quad 6479.7485 \quad 7837.4990 \quad 1.2095375 \quad-15.24870 \quad-4.27001-71.64000$

$\begin{array}{lllllll}1542 & 4080.1113 & 2943.3879 & 0.7213989 & -19.58683 & -9.64501 & -66.25000\end{array}$

$\begin{array}{lllllll}1546 & 729.3591 & 892.3582 & 1.2234826 & -23.92366 & -9.64501 & -77.03100\end{array}$

$1547 \quad 888.4908 \quad 1106.3942 \quad 1.2452511 \quad-19.58683 \quad-9.64501 \quad-77.03100$

$\begin{array}{lllllll}1548 & 512.0069 & 643.9552 & 1.2577081 & -19.58683 & -9.64501 & -71.64000\end{array}$

$\begin{array}{lllllll}1588 & 10556.6924 & 7633.0361 & 0.7230518 & -15.24827 & -2.47834 & -66.25000\end{array}$

$\begin{array}{lllllll}1594 & 6707.6372 & 4977.5317 & 0.7420693 & -23.93646 & -5.51314 & -66.25000\end{array}$

$\begin{array}{llllllll}1595 & 4904.3843 & 3568.8181 & 0.7276791 & -19.59871 & -5.81784 & -66.25000\end{array}$

$1599 \quad 10249.5762 \quad 6960.7695 \quad 0.6791276-19.58683-13.94750-66.25000$ 


\section{SECTION A-A}

$\begin{array}{rrrrrrrr}\text { NOOE } & \text { STATIC } & \text { FAILURE } & \text { FACTOR } & X \text {-LOC } & Y \text {-LOC } & \text { ZLOC } \\ 14 & 12598.3779 & 11791.8184 & 0.9359791 & 19.62247 & 1.83647 & 0.00000 \\ 15 & 9870.0508 & 10115.6328 & 1.0248815 & 23.96206 & 2.75057 & 0.00000 \\ 113 & 6672.7446 & 8348.7227 & 1.2511678 & 15.24870 & -4.27001 & 0.00000 \\ 126 & 5887.9609 & 5804.4741 & 0.9858208 & 28.26048 & -5.14798 & 0.00000 \\ 127 & 5448.1396 & 5680.3828 & 1.0426279 & 28.26047 & -0.65095 & 0.00000 \\ 142 & 3789.2974 & 2753.1521 & 0.7265601 & 19.58683 & -9.64501 & 0.00000 \\ 143 & 6419.9321 & 5027.4707 & 0.7831034 & 23.92366 & -9.64501 & 0.00000 \\ 188 & 9572.9775 & 6707.5996 & 0.7006806 & 15.24827 & -2.47834 & 0.00000 \\ 189 & 1850.8263 & 2622.8689 & 1.4171340 & 15.24913 & -6.06167 & 0.00000\end{array}$

QUADRANT 2

NODE STATIC FAILURE FACTOR X-LOC Y-LOC ZLOC

$\begin{array}{llllllll}414 & 13735.7178 & 14714.0322 & 1.0712241 & 19.62247 & 1.83647 & -66.25000\end{array}$

$\begin{array}{llllllll}415 & 10490.8438 & 10334.7891 & 0.985 ! 246 & 23.96206 & 2.75057 & -66.25000\end{array}$

$\begin{array}{llllllll}513 & 6620.7393 & 5340.4595 & 0.8066258 & 15.24870 & -4.27001 & -66.25000\end{array}$

$\begin{array}{lllllll}526 & 6102.7935 & 6120.2676 & 1.0028633 & 28.26048 & -5.14798 & -66.25000\end{array}$

$\begin{array}{llllllll}527 & 5643.4819 & 5403.8066 & 0.9575306 & 28.26047 & -0.65095 & -66.25000\end{array}$

$\begin{array}{lllllll}542 & 4080.1113 & 5856.6660 & 1.4354182 & 19.58683 & -9.64501 & -66.25000\end{array}$

$\begin{array}{llllllll}543 & 6830.1108 & 8072.9741 & 1.1819682 & 23.92366 & -9.64501 & -66.25000\end{array}$

$\begin{array}{llllllll}588 & 10556.6924 & 13547.4023 & 1.2832999 & 15.24827 & -2.47834 & -66.25000\end{array}$

$\begin{array}{lllllll}589 & 2271.6011 & 4967.1338 & 2.1866224 & 15.24913 & -6.06167 & -66.25000\end{array}$ 
NODE STATIC FAILURE FACTOR X-LOC Y-LOC ZLOC

$\begin{array}{llllllll}1014 & 12598.3779 & 13433.9111 & 1.0663207 & -19.62247 & 1.83647 & 0.00000\end{array}$

$\begin{array}{lllllll}1015 & 9870.0508 & 9751.8311 & 0.9880224 & -23.96206 & 2.75057 & 0.00000\end{array}$

$\begin{array}{lllllll}1113 & 6672.7446 & 5287.7793 & 0.7924444 & -15.24870 & -4.27001 & 0.00000\end{array}$

$\begin{array}{lllllll}1126 & 5887.9609 & 6017.7896 & 1.0220498 & -28.26048 & -5.14798 & 0.00000\end{array}$

$\begin{array}{lllllll}1127 & 5448.1396 & 5254.7271 & 0.9644994 & -28.26047 & -0.65095 & 0.00000\end{array}$

$\begin{array}{lllllll}1142 & 3789.2974 & 5674.7534 & 1.4975741 & -19.58683 & -9.64501 & 0.00000\end{array}$

$\begin{array}{lllllll}1143 & 6419.9321 & 7842.2515 & 1.2215474 & -23.92366 & -9.64501 & 0.00000\end{array}$

$\begin{array}{lllllll}1188 & 9572.9775 & 12650.5313 & 1.3214835 & -15.24827 & -2.47834 & 0.00000\end{array}$

$\begin{array}{lllllll}1189 & 1850.8263 & 4745.8682 & 2.5641890 & -15.24913 & -6.06167 & 0.00000\end{array}$

QUADRANT 4

NODE STATIC FAILURE FACTOR X-LOC Y-LOC ZLOC

$\begin{array}{lllllll}1414 & 13735.7178 & 12782.0352 & 0.9305692 & -19.62247 & 1.83647 & -66.25000\end{array}$

$\begin{array}{llllllll}1415 & 10490.8438 & 10738.1572 & 1.0235742 & -23.96206 & 2.75057 & -66.25000\end{array}$

$\begin{array}{llllllll}1513 & 6620.7393 & 8125.8618 & 1.2273345 & -15.24870 & -4.27001 & -66.25000^{-}\end{array}$

$\begin{array}{lllllll}1526 & 6102.7935 & 6107.2676 & 1.0007331 & -28.26048 & -5.14798 & -66.25000\end{array}$

$\begin{array}{lllllll}1527 & 5643.4819 & 5909.6851 & 1.0471700 & -28.26047 & -0.65095 & -66.25000\end{array}$

$\begin{array}{lllllll}1542 & 4080.1113 & 2943.3879 & 0.7213989 & -19.58683 & -9.64501 & -66.25000\end{array}$

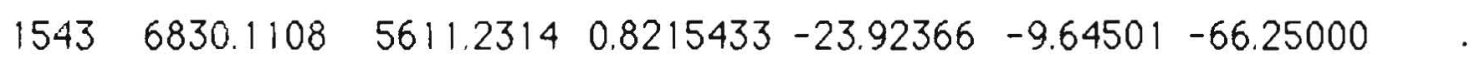

$\begin{array}{lllllll}1588 & 10556.6924 & 7633.0361 & 0.7230518 & -15.24827 & -2.47834 & -66.25000\end{array}$

$\begin{array}{lllllll}1589 & 2271.6011 & 2420.1196 & 1.0653806 & -15.24913 & -6.06167 & -66.25000\end{array}$ 


\section{SECTION B-B}

NODE STATIC FAILURE FACTOR $X$-LOC $y$-LOC ZLOC

$\begin{array}{lllllll}75 & 1214.2780 & 5037.9116 & 4.1488948 & 15.24740 & 1.10498 & -33.12500\end{array}$

$\begin{array}{llllllll}87 & 4162.9565 & 4641.5479 & 1.1149642 & 28.26045 & 3.84607 & -33.12500\end{array}$

$90 \quad 3170.8860 \quad 4314.3813 \quad 1.3606232 \quad 23.96206 \quad 2.75057 \quad-33.12500$

$\begin{array}{llllllll}91 & 2845.5210 & 5009.4805 & 1.7604792 & 19.62247 & 1.83647 & -33.12500\end{array}$

$\begin{array}{lllllll}96 & 5683.3965 & 6317.6992 & 1.1116062 & 31.97561 & 4.95008 & -33.12500\end{array}$

$\begin{array}{lllllll}216 & 913.7531 & 1047.6416 & 1.1465260 & 15.25000 & -18.25000 & -33.12500\end{array}$

$219 \quad 2392.3420 \quad 2645.5015 \quad 1.1058208 \quad 0.00000-18.25000-33.12500$

$\begin{array}{lllllll}220 & 1392.2172 & 1575.4454 & 1.1316090 & 10.16666 & -18.25000 & -33.12500\end{array}$

$\begin{array}{lllllll}221 & 2134.5977 & 2366.6130 & 1.1086928 & 5.08333 & -18.25000 & -33.12500\end{array}$

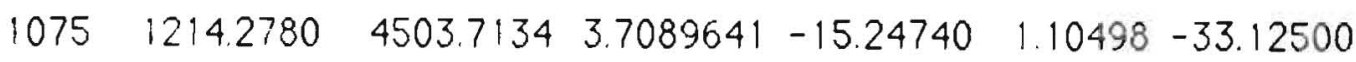

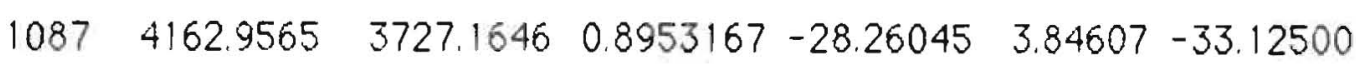

$1090 \quad 3170.8860 \quad 2759.7566 \quad 0.8703424-23.96206 \quad 2.75057-33.12500$

$10912845.5210 \quad 2910.4431 \quad 1.0228156-19.62247 \quad 1.83647-33.12500$

$1096 \quad 5683.3965 \quad 5+19.51610 .9007846-31.97561 \quad 4.95008-33.12500$

$\begin{array}{lllllll}1216 & 913.7531 & 1119.7089 & 1.2253954 & -15.25000 & -18.25000 & -33.12500\end{array}$

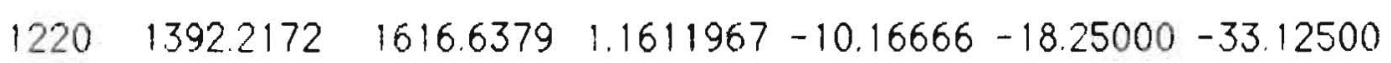

$\begin{array}{lllllll}1221 & 2134.5977 & 2389.9517 & 1.1196263 & -5.08333 & -18.25000 & -33.12500\end{array}$

$12425220.9829 \quad 5264.5967 \quad 1.0083536-23.92366-18.25000-33.12500$

$1243 \quad 2415.5967 \quad 2485.2119 \quad 1.0288191-19.58683-18.25000-33.12500$ 


\section{ARM CONNECT}

NODE STATIC FAILURE FACTOR X-LOC Y-LOC ZLOC

$\begin{array}{llllllll}121 & 15304.7686 & 15814.9473 & 1.0333346 & 28.26050 & -9.64501 & -11.25000\end{array}$

$\begin{array}{lllllll}124 & 9821.5498 & 9683.4590 & 0.9859400 & 28.26050 & -9.64501 & 0.00000\end{array}$

$125 \quad 5693.9976 \quad 6040.4214 \quad 1.0608401 \quad 28.26050 \quad-9.64501 \quad-5.62500$

$\begin{array}{lllllll}181 & 10963.5166 & 10026.1689 & 0.9145030 & 28.26050 & -18.25000 & 0.00000\end{array}$

$\begin{array}{lllllll}182 & 13214.3350 & 12588.0078 & 0.9526024 & 28.26050 & -13.94750 & 0.00000\end{array}$

$\begin{array}{lllllll}228 & 16970.1816 & 18126.7520 & 1.0681531 & 28.26050 & -18.25000 & -11.25000\end{array}$

$2319056.7744 \quad 9069.7773 \quad 1.0014358 \quad 28.26050-18.25000 \quad-5.62500$

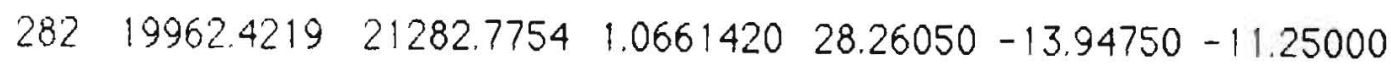

$\begin{array}{lllllll}305 & 7261.1777 & 7576.2388 & 1.0433898 & 28.26050 & -13.94750 & -5.62500\end{array}$

QUADRANT 2

NODE STATIC FAILURE FACTOR X-LOC Y-LOC ZLOC

$\begin{array}{llllllll}521 & 14896.8818 & 14559.9443 & 0.9773820 & 28.26050 & -9.64501 & -55.00000\end{array}$

$\begin{array}{lllllll}-524 & 10119.7666 & 10144.3369 & 1.0024279 & 28.26050 & -9.64501 & -66.25000\end{array}$

$\begin{array}{llllllll}525 & 5833.1396 & 5524.3052 & 0.9470552 & 28.26050 & -9.64501 & -60.62500\end{array}$

$\begin{array}{llllllll}581 & 11380.4199 & 12200.0381 & 1.0720201 & 28.26050 & -18.25000 & -66.25000\end{array}$

$\begin{array}{lllllll}582 & 13696.2100 & 14132.6904 & 1.0318687 & 28.26050 & -13.94750 & -66.25000\end{array}$

$\begin{array}{lllllll}628 & 16811.6035 & 15628.7354 & 0.9296398 & 28.26050 & -18.25000 & -55.00000\end{array}$

$631 \quad 9250.3848 \quad 9182.5303 \quad 0.9926647 \quad 28.26050-18.25000-60.62500$

$\begin{array}{lllllll}682 & 19503.0332 & 18310.3184 & 0.9388446 & 28.26050 & -13.94750 & -55.00000\end{array}$

$\begin{array}{lllllll}705 & 7568.0835 & 7375.0459 & 0.9744932 & 28.26050 & -13.94750 & -60.62500\end{array}$ 


\section{QUADRANT 3}

$\begin{array}{lllllll}\text { NODE } & \text { STATIC } & \text { FAILURE } & \text { FACTOR } & \text { X-LOC } & \text { Y-LOC } & \text { ZLOC } \\ 1121 & 15304.7686 & 14825.1855 & 0.9686645 & -28.26050 & -9.64501 & -11.25000 \\ 1124 & 9821.5498 & 9985.7168 & 1.0167149 & -28.26050 & -9.64501 & 0.00000 \\ 1125 & 5693.9976 & 5445.6196 & 0.9563790 & -28.26050 & -9.64501 & -5.62500 \\ 1181 & 10963.5166 & 11917.9160 & 1.0870523 & -28.26050 & -18.25000 & 0.00000 \\ 1182 & 13214.3350 & 13858.9023 & 1.0487779 & -28.26050 & -13.94750 & 0.00000 \\ 1228 & 16970.1816 & 15819.4238 & 0.9321894 & -28.26050 & -18.25000 & -11.25000 \\ 1231 & 9056.7744 & 9068.8096 & 1.0013288 & -28.26050 & -18.25000 & -5.62500 \\ 1282 & 19962.4219 & 18662.9531 & 0.9349043 & -28.26050 & -13.94750 & -11.25000 \\ 1305 & 7261.1777 & 7176.5845 & 0.9883499 & -28.26050 & -13.94750 & -5.62500\end{array}$

QUADRANT 4

NODE STATIC FAILURE FACTOR X-LOC Y-LOC ZLOC

$\begin{array}{lllllll}1521 & 14896.8818 & 15276.3467 & 1.0254728 & -28.26050 & -9.64501 & -55.00000\end{array}$

$\begin{array}{lllllll}1524 & 10119.7666 & 10117.9736 & 0.9998228 & -28.26050 & -9.64501 & -66.25000\end{array}$

$1525^{-} 5833.1396 \quad 6255.3540 \quad 1.0723820-28.26050-9.64501-60.62500$

$1581 \quad 11380.4199 \quad 10590.0791 \quad 0.9305526-28.26050-18.25000-66.25000$

$\begin{array}{lllllll}1582 & 13696.2100 & 13278.0430 & 0.9694684 & -28.26050 & -13.94750 & -66.25000\end{array}$

$\begin{array}{lllllll}1628 & 16811.6035 & 17999.1348 & 1.0706376 & -28.26050 & -18.25000 & -55.00000\end{array}$

$16319250.3848 \quad 9355.4053 \quad 1.0113531-28.26050-18.25000 \quad-60.62500$

$\begin{array}{lllllll}1682 & 19503.0332 & 20744.4395 & 1.0636519 & -28.26050 & -13.94750 & -55.00000\end{array}$

$\begin{array}{llllllll}1705 & 7568.0835 & 8038.0371 & 1.0620968 & -28.26050 & -13.94750 & -60.62500\end{array}$ 


\section{TIE PLATE}

NODE STATIC FAILURE FACTOR X-LOC Y-LOC ZLOC

$1 \quad \begin{array}{llllll}19640.0820 & 19644.3984 & 1.0002198 & 0.41207 & 0.00000 & 0.00000\end{array}$

$\begin{array}{lllllll}7 & 15607.1484 & 15718.1660 & 1.0071132 & 0.41207 & 0.00000 & -11.25000\end{array}$

$\begin{array}{llllllll}10 & 18799.2285 & 18825.8184 & 1.0014144 & 0.41207 & 0.00000 & -5.62500\end{array}$

$51 \quad 8697.8447 \quad 9168.4990 \quad 1.0541116 \quad 0.41207 \quad 0.00000-19.12500$

$\begin{array}{lllllll}54 & 12335.9551 & 12610.5342 & 1.0222584 & 0.41207 & 0.00000 & -15.18700\end{array}$

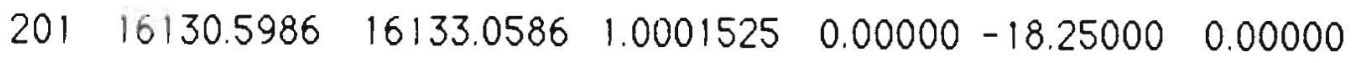

$206 \quad 9685.4326 \quad 9724.5205 \quad 1.0040357 \quad 0.00000-18.25000-11.25000$

$\begin{array}{lllllll}209 & 13084.1748 & 13096.5195 & 1.0009435 & 0.00000 & -18.25000 & -5.62500\end{array}$

$2126461.3804 \quad 6542.1323 \quad 1.0124977 \quad 0.00000-18.25000-16.62500$

$219 \quad 2392.3420 \quad 2645.5015 \quad 1.1058208 \quad 0.00000-18.25000-33.12500$

$\begin{array}{lllllll}222 & 2305.1980 & 2715.9443 & 1.1781827 & 0.00000 & -18.25000 & -27.62500\end{array}$

$\begin{array}{lllllll}223 & 3737.9561 & 3923.7046 & 1.0496925 & 0.00000 & -18.25000 & -22.12500\end{array}$

$\begin{array}{lllllll}401 & 18701.4219 & 18726.4688 & 1.0013393 & 0.41207 & 0.00000 & -66.25000\end{array}$

$\begin{array}{lllllll}407 & 19952.5508 & 20054.6563 & 1.0051174 & 0.41207 & 0.00000 & -55.00000\end{array}$

$\begin{array}{llllllll}410 & 20354.2109 & 20427.5723 & 1.0036043 & 0.41207 & 0.00000 & -60.62500\end{array}$

$\begin{array}{lllllll}454 & 26097.8770 & 26143.9609 & 1.0017658 & 0.41207 & 0.00000 & -51.06200\end{array}$

$\begin{array}{lllllll}601 & 16349.8105 & 16351.6484 & 1.0001124 & 0.00000 & -18.25000 & -66.25000\end{array}$

$\begin{array}{lllllll}606 & 9756.7295 & 9788.5986 & 1.0032663 & 0.00000 & -18.25000 & -55.00000\end{array}$

$\begin{array}{lllllll}609 & 13253.6445 & 13263.2666 & 1.0007260 & 0.00000 & -18.25000 & -60.62500\end{array}$

$\begin{array}{lllllll}612 & 6468.4209 & 6538.3867 & 1.0108166 & 0.00000 & -18.25000 & -49.62500\end{array}$

$\begin{array}{lllllll}622 & 2322.6453 & 2715.5752 & 1.1691735 & 0.00000 & -18.25000 & -38.62500\end{array}$

$\begin{array}{lllllll}623 & 3720.1455 & 3894.8093 & 1.0469508 & 0.00000 & -18.25000 & -44.12500\end{array}$ • 


\section{STANCHION AND 3.5 in SUPPORT PLATE}

NODE STATIC FAILURE FACTOR X-LOC Y-LOC ZLOC

$\begin{array}{llllllll}38 & 20611.9492 & 20601.4395 & 0.9994901 & 66.62949 & 23.63070 & -5.62500\end{array}$

$\begin{array}{lllllll}100 & 8643.2266 & 8670.5479 & 1.0031610 & 79.90000 & 23.63070 & 0.00000\end{array}$

$101 \quad 11539.8076 \quad 11460.6406 \quad 0.9931397 \quad 71.05299 \quad 23.63070 \quad 0.00000$

$\begin{array}{lllllll}102 & 10073.4092 & 10036.0547 & 0.9962918 & 75.47649 & 23.63070 & 0.00000\end{array}$

$103 \quad 5573.9229 \quad 5589.1187 \quad 1.002726279 .90000 \quad 23.63070-11.25000$

$104 \quad 13872.4805 \quad 13885.1934 \quad 1.0009164 \quad 79.90000 \quad 23.63070 \quad-5.62500$

$105 \quad 7140.9375 \quad 7172.3394 \quad 1.004397475 .47649 \quad 23.63070-11.25000$

$\begin{array}{lllllll}106 & 9418.0049 & 9440.0400 & 1.0023397 & 71.05299 & 23.63070 & -11.25000\end{array}$

$107 \quad 20541.1270 \quad 20538.4902 \quad 0.9998716 \quad 71.0529923 .63070 \quad-5.62500$

$\begin{array}{llllllll}108 & 14999.4717 & 14999.2900 & 0.9999879 & 75.47649 & 23.63070 & -5.62500\end{array}$

$\begin{array}{llllllll}111 & 9475.7090 & 10692.2480 & 1.1283851 & 15.25000 & -9.64501 & 0.00000\end{array}$

$\begin{array}{lllllll}124 & 12426.9697 & 12405.5225 & 0.9982741 & 28.26050 & -9.64501 & 0.00000\end{array}$

$\begin{array}{llllllll}138 & 3173.0437 & 3529.7180 & 1.1124077 & 15.25000 & -9.64501 & 10.78100\end{array}$

$\begin{array}{lllllll}139 & 83 \overline{30} .6182 & 8975.0898 & 1.0773618 & 15.25000 & -9.64501 & 5.39050\end{array}$

$\begin{array}{lllllll}142 & 8351.6768 & 8982.9365 & 1.0755848 & 19.58683 & -9.64501 & 0.00000\end{array}$

$\begin{array}{llllllll}143 & 9834.9131 & 10144.9697 & 1.0315261 & 23.92366 & -9.64501 & 0.00000\end{array}$

$\begin{array}{llllllll}444 & 2550.2729 & 2502.7712 & 0.9813738 & 28.26050 & -9.64501 & 10.78100\end{array}$

$\begin{array}{lllllll}145 & 6295.2349 & 7411.9106 & 1.1773843 & 28.26050 & -9.64501 & 5.39050\end{array}$

$\begin{array}{lllllll}146 & 11103.1084 & 12900.5859 & 1.1618896 & 23.92366 & -9.64501 & 10.78100\end{array}$

$147 \quad 11455.2148 \quad 13455.4805 \quad 1.1746162 \quad 19.58683 \quad-9.64501 \quad 10.78100$

$\begin{array}{lllllll}148 & 11864.2979 & 13876.7324 & 1.1696210 & 19.58683 & -9.64501 & 5.39050\end{array}$ 
QUADRANT 2

NODE STATIC FAILURE FACTOR $X$-LOC Y-LOC ZLOC

$\begin{array}{lllllll}438 & 20613.4004 & 20623.7344 & 1.0005013 & 66.62949 & 23.63070 & -60.62500\end{array}$

$\begin{array}{lllllll}500 & 8634.4326 & 8617.4297 & 0.9980308 & 79.90000 & 23.63070 & -66.25000\end{array}$

$\begin{array}{lllllll}501 & 11559.0742 & 11621.0313 & 1.0053600 & 71.05299 & 23.63070 & -66.25000\end{array}$

$\begin{array}{lllllll}502 & 10083.4600 & 10110.8447 & 1.0027158 & 75.47649 & 23.63070 & -66.25000\end{array}$

$\begin{array}{lllllll}503 & 5570.0698 & 5560.5732 & 0.9982951 & 79.90000 & 23.63070 & -55.00000\end{array}$

$\begin{array}{lllllll}504 & 13869.1133 & 13860.7451 & 0.9993966 & 79.90000 & 23.63070 & -60.62500\end{array}$

$\begin{array}{lllllll}505 & 7129.6475 & 7112.3633 & 0.9975758 & 75.47649 & 23.63070 & -55.00000\end{array}$

$\begin{array}{lllllll}506 & 9418.7100 & 9391.2734 & 0.9970870 & 71.05299 & 23.63070 & -55.00000\end{array}$

$\begin{array}{lllllll}507 & 20541.9375 & 20542.8926 & 1.0000465 & 71.05299 & 23.63070 & -60.62500\end{array}$

$\begin{array}{lllllll}508 & 14999.5244 & 14999.2148 & 0.9999794 & 75.47649 & 23.63070 & -60.62500\end{array}$

$\begin{array}{lllllll}511 & 9637.7139 & 9562.7676 & 0.9922236 & 15.25000 & -9.64501 & -66.25000\end{array}$

$\begin{array}{lllllll}524 & 12829.6514 & 12708.9414 & 0.9905913 & 28.26050 & -9.64501 & -66.25000\end{array}$

$\begin{array}{lllllll}538 & 3232.4375 & 3300.4346 & 1.0210358 & 15.25000 & -9.64501 & -77.03100\end{array}$

$\begin{array}{llllllll}539 & 8546.4316 & 8086.9478 & 0.9462367 & 15.25000 & -9.54501 & -71.64000\end{array}$

$\begin{array}{lllllll}542 & 8571.9434 & 8737.6299 & 1.0193290 & 19.58683 & -9.54501 & -66.25000\end{array}$

$\begin{array}{lllllll}543 & 10170.0645 & 10651.5283 & 1.0473412 & 23.92366 & -9.64501 & -66.25000\end{array}$

$\begin{array}{lllllll}544 & 2739.4868 & 2949.8948 & 1.0768056 & 28.26050 & -9.64501 & -77.03100\end{array}$

$\begin{array}{lllllll}545 & 6406.6108 & 5852.2310 & 0.9134675 & 28.26050 & -9.64501 & -71.64000\end{array}$

$\begin{array}{llllllll}546 & 11204.1172 & 9378.0381 & 0.8370171 & 23.92366 & -9.64501 & -77.03100\end{array}$

$\begin{array}{llllllll}547 & 11401.9746 & 9438.3428 & 0.8277814 & 19.58683 & -9.64501 & -77.03100\end{array}$

$\begin{array}{llllllll}548 & 11870.9609 & 9863.2178 & 0.8308694 & 19.58683 & -9.64501 & -71.64000\end{array}$

$\begin{array}{lllllll}549 & 11699.2109 & 9691.1621 & 0.8283603 & 23.92366 & -9.64501 & -71.64000\end{array}$ 
NODE STATIC FAILURE FACTOR X-LOC Y-LOC ZLOC

$1038 \quad 20611.9492 \quad 20622.5098 \quad 1.0005124 \quad-66.62949 \quad 23.63070 \quad-5.62500$

$\begin{array}{lllllll}1100 & 8643.2266 & 8616.0234 & 0.9968526 & -79.90000 & 23.63070 & 0.00000\end{array}$

$\begin{array}{lllllll}1101 & 11539.8076 & 11619.3447 & 1.0068924 & -71.05299 & 23.63070 & 0.00000\end{array}$

$\begin{array}{lllllll}1102 & 10073.4092 & 10110.9346 & 1.0037252 & -75.47649 & 23.63070 & 0.00000\end{array}$

$1103 \quad 5573.9229 \quad 5560.5020 \quad 0.9975922 \quad-79.90000 \quad 23.63070-11.25000$

$\begin{array}{lllllll}1104 & 13872.4805 & 13860.6553 & 0.9991476 & -79.90000 & 23.63070 & -5.62500\end{array}$

$\begin{array}{lllllll}1105 & 7140.9375 & 7109.9888 & 0.9956660 & -75.47649 & 23.63070 & -11.25000\end{array}$

$\begin{array}{lllllll}1106 & 9418.0049 & 9396.2188 & 0.9976867 & -71.05299 & 23.63070 & -11.25000\end{array}$

$\begin{array}{llllllll}1107 & 20541.1270 & 20543.7930 & 1.0001298 & -71.05299 & 23.63070 & -5.62500\end{array}$

$\begin{array}{llllllll}1108 & 14999.4717 & 14999.7148 & 1.0000162 & -75.47649 & 23.63070 & -5.62500\end{array}$

$\begin{array}{llllllll}1111 & 9475.7090 & 9222.7266 & 0.9733020 & -15.25000 & -9.64501 & 0.00000\end{array}$

$\begin{array}{lllllll}1124 & 12426.9697 & 12482.3721 & 1.0044582 & -28.26050 & -9.64501 & 0.00000\end{array}$

$1138 \quad 3173.0437 \quad 3155.4670 \quad 0.9944606-15.25000 \quad-9.64501 \neq 0.78100$

$1139 \quad 8330.6182 \quad 7861.4546 \quad 0.9436820-15.25000 \quad-9.64501 \quad 5.39050$

$\begin{array}{lllllll}1142 & 8351.6768 & 8528.2871 & 1.0211467 & -19.58683 & -9.64501 & 0.00000\end{array}$

$\begin{array}{lllllll}1143 & 9834.9131 & 10431.3623 & 1.0606461 & -23.92366 & -9.64501 & 0.00000\end{array}$

$\begin{array}{lllllll}1144 & 2550.2729 & 2782.8181 & 1.0911844 & -28.26050 & -9.64501 & 10.78100\end{array}$

$1145 \quad 6295.2349 \quad 5734.3325 \quad 0.9109005 \quad-28.26050 \quad-9.64501 \quad 5.39050$

$\begin{array}{lllllll}1146 & 11103.1084 & 9311.4326 & 0.8386329 & -23.92366 & -9.64501 & 10.78100\end{array}$

$\begin{array}{lllllll}1147 & 11455.2148 & 9456.7842 & 0.8255440 & -19.58683 & -9.64501 & 10.78100\end{array}$

$\begin{array}{lllllll}1148 & 11864.2979 & 9854.3691 & 0.8305902 & -19.58683 & -9.64501 & 5.39050\end{array}$

$\begin{array}{lllllll}1149 & 11676.6074 & 9671.9199 & 0.8283159 & -23.92366 & -9.64501 & 5.39050\end{array}$ 


\section{QUADRANT 4}

NODE STATIC FAILURE FACTOR X-LOC Y-LOC ZLOC

$\begin{array}{lllllll}1438 & 20613.4004 & 20603.0781 & 0.9994993 & -66.62949 & 23.63070 & -60.62500\end{array}$

$\begin{array}{lllllll}1500 & 8634.4326 & 8651.5156 & 1.0019785 & -79.90000 & 23.63070 & -66.25000\end{array}$

$\begin{array}{lllllll}1501 & 11559.0742 & 11497.3867 & 0.9946633 & -71.05299 & 23.63070 & -66.25000\end{array}$

$\begin{array}{lllllll}1502 & 10083.4600 & 10056.1709 & 0.9972937 & -75.47649 & 23.63070 & -66.25000\end{array}$

$1503 \quad 5570.0698 \quad 5580.3613 \quad 1.0018476 \quad-79.90000 \quad 23.63070-55.00000$

$\begin{array}{lllllll}1504 & 13869.1133 & 13877.9121 & 1.0006344 & -79.90000 & 23.63070 & -60.62500\end{array}$

$\begin{array}{lllllll}1505 & 7129.6475 & 7147.0601 & 1.0024422 & -75.47649 & 23.63070 & -55.00000\end{array}$

$\begin{array}{lllllll}1506 & 9418.7100 & 9446.2285 & 1.0029217 & -71.05299 & 23.63070 & -55.00000\end{array}$

$\begin{array}{lllllll}1507 & 20541.9375 & 20540.9922 & 0.9999540 & -71.05299 & 23.63070 & -60.62500\end{array}$

$\begin{array}{lllllll}1508 & 14999.5244 & 14999.8369 & 1.0000209 & -75.47649 & 23.63070 & -60.62500\end{array}$

$\begin{array}{lllllll}1511 & 9637.7139 & 10489.1299 & 1.0883421 & -15.25000 & -9.64501 & -66.25000\end{array}$

$\begin{array}{lllllll}1524 & 12829.6514 & 12983.3398 & 1.0119791 & -28.26050 & -9.64501 & -66.25000\end{array}$

$\begin{array}{llllllll}1538 & 3232.4375 & 3446.4961 & 1.0662221 & -15.25000 & -9.64501 & -77.03100\end{array}$

$\begin{array}{lllllll}1539 & 8546.4316 & 9206.3154 & 1.0772116 & -15.25000 & -9.64501 & -71.64000\end{array}$

$\begin{array}{llllllll}1542 & 8571.9434 & 9083.2158 & 1.0596448 & -19.58683 & -9.64501 & -66.25000\end{array}$

$\begin{array}{llllllll}1543 & 10170.0645 & 10356.4727 & 1.0183291 & -23.92366 & -9.64501 & -66.25000\end{array}$

$\begin{array}{lllllll}1544 & 2739.4868 & 2696.3022 & 0.9842362 & -28.26050 & -9.64501 & -77.03100\end{array}$

$1545 \quad 6406.6108 \quad 7287.0957 \quad 1.1374338-28.26050 \quad-9.64501-71.64000$

$1546 \quad 11204.1172 \quad 130345352 \quad 1.1633701 \quad-23.92366 \quad-9.64501 \quad-77.03100$

$\begin{array}{lllllll}1547 & 11401.9746 & 13366.8760 & 1.1723299 & -19.58683 & -9.64501 & -77.03100\end{array}$

$\begin{array}{lllllll}1548 & 11870.9609 & 13880.4238 & 1.1692755 & -19.58683 & -9.64501 & -71.64000\end{array}$

$\begin{array}{lllllll}1549 & 11699.2109 & 13713.2676 & 1.1721532 & -23.92366 & -9.64501 & -71.64000\end{array}$ 
APPENDIX :

HAND CALCULATIONS 


\section{Failure Stress Calculations}

For all stress hand calculations for the Jack Failure Analysis of the Central Calorimeter Cradle, the most severe reaction force will be used along with Leo Engstrom's stress calculations from Engineering Note $3740.251-E N-14$, which also contains all moment of inertia and static case calculations.

These diagrams will be referred to in all five cases and are defined in the above mentioned Engineering Note.

\section{Central Beam}

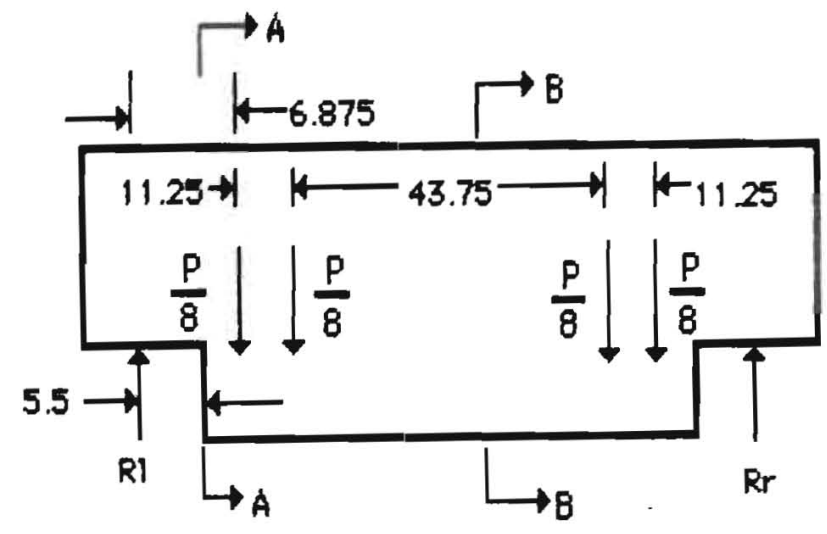

Figure 9

Section A-A

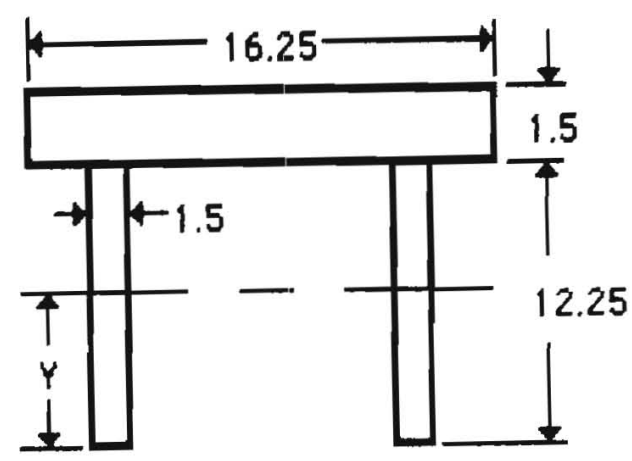

Figure 10 
Section B-B

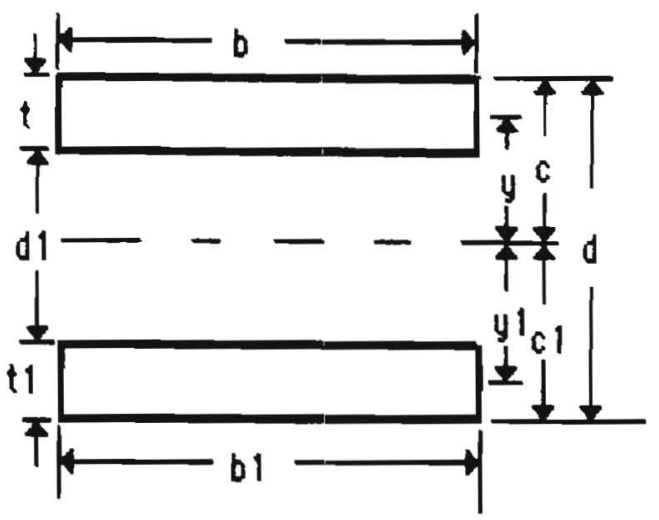

Figure 11

Section A-A B-B Shear

\section{Arm}

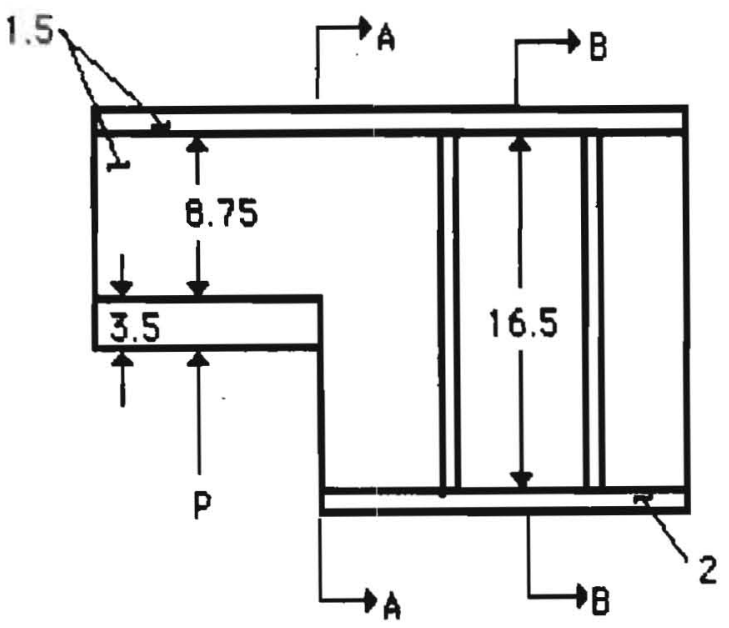

Figure 12

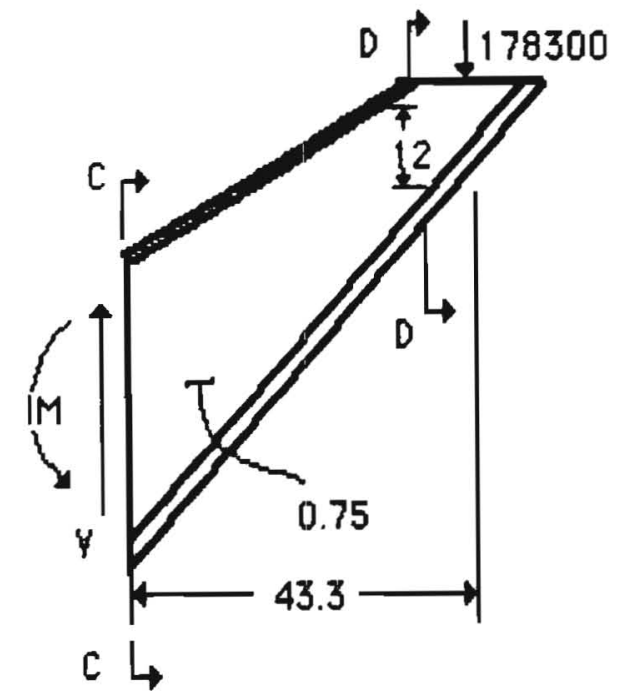

Figure 13 
Tie Plate

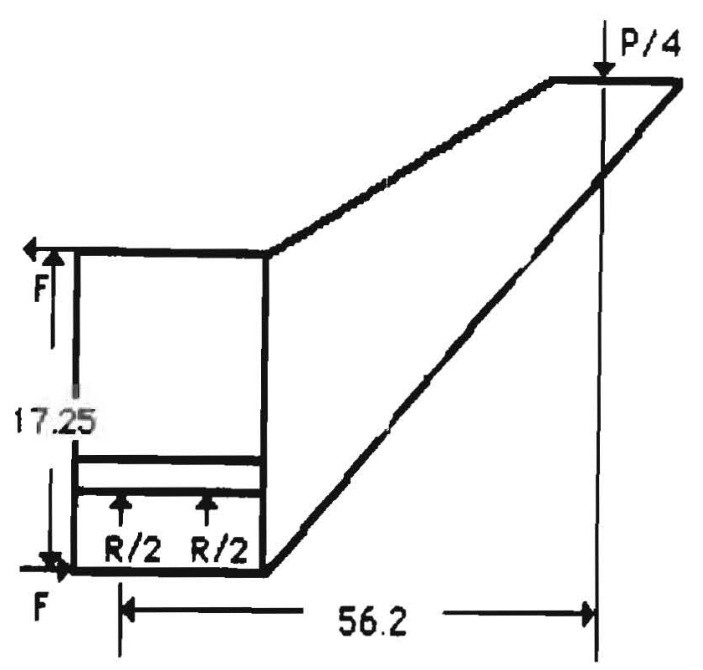

Figure 14

Stanchion Plate

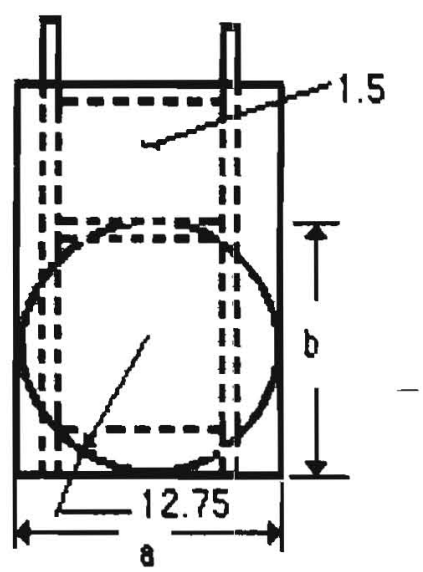

Figure 15

3.5 in Bearing Plate

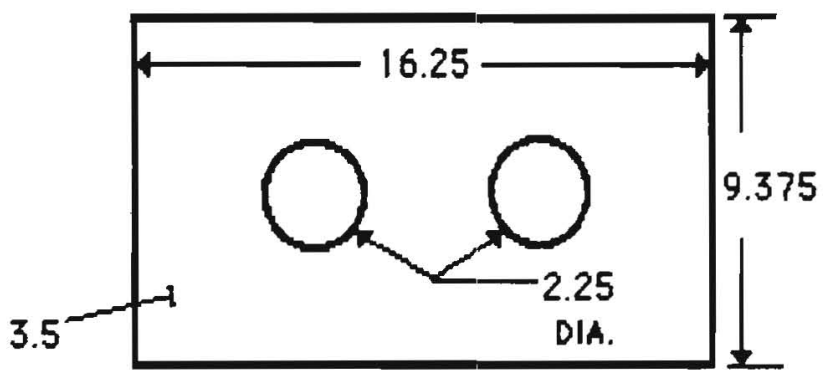

Figure 16 
Number One

Stress

$$
m_{\max }=2228.75 \mathrm{kipsin} \quad V_{\max }=186.646 \mathrm{klps}
$$

Bending Stress

In Section B-B - Figure 11 -

$$
\begin{aligned}
& c=11.179 \mathrm{in} ; c=8.821 ; d=20^{\prime \prime} \\
& I=4654 \mathrm{in}^{4}-\text { from Engineering Note } 3740.215-\text { EN .14 } \\
& \sigma=\frac{m_{\max } c}{I}=\frac{2228.75 \times 10^{+3}(11.179)}{4654}=5.354 \mathrm{psi}
\end{aligned}
$$

In section $A-A$ - Figure 10 .

$$
\begin{aligned}
y=8.867-I= & 1157 \mathrm{~min}^{4} \\
m_{\text {max }}=R(d) & =186,646(5.5) \\
& =10.2655 \times 10^{6} \mathrm{in} \mathrm{b} \\
\sigma_{\max }=\frac{m_{\max } C}{I} & =\frac{10.2655 \times 10^{6}(8.867)}{1157} \\
& =\frac{7867.28 \mathrm{ps}}{}
\end{aligned}
$$

Shear Stress

shear between webs - Section B-B of Figure 12

$$
V=102596 \mathrm{lbs}
$$

$$
T=V / A=\frac{102596}{2(1.5)(16.5)}=2072.646 \mathrm{psi}
$$

Shear at section $A-A$ of Figure 12

$$
\begin{aligned}
& P=186646 \mathrm{~kb} \\
& T=P / A=\frac{186646}{2(1.5)(8.75)}=7110.32 p s i
\end{aligned}
$$

Arms

Since the load on the Arms do not vary, the stresses will be exactly the same as the static case 
\begin{tabular}{l|l} 
Number One Stress &
\end{tabular}

Stanchion Plate Since no variation in Armload, Stanchion plate
stresses will not change from the static case.

$3 \%$ "Bearing Plate Wong Figure 16 and drawing Freebody, shear ard
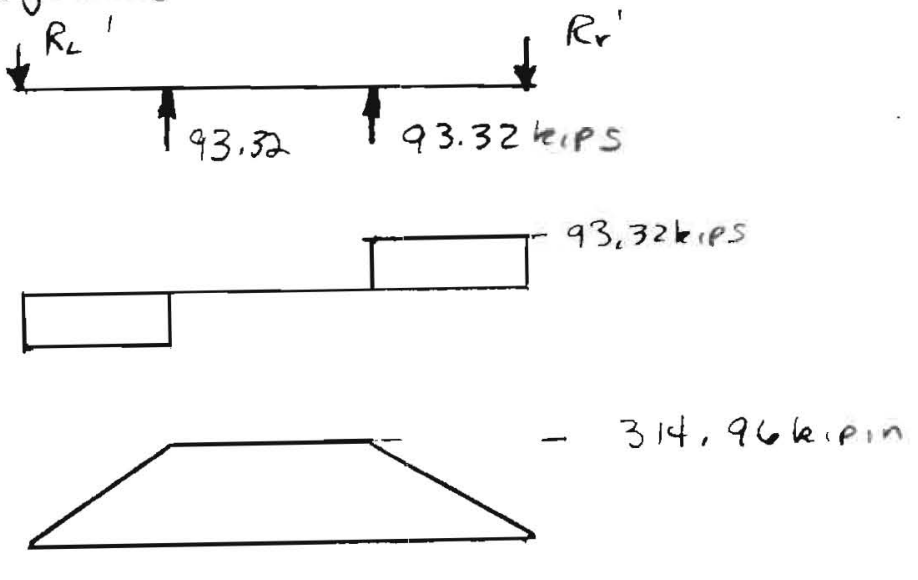

Bending

$$
\begin{aligned}
& m_{\max }=314.96 \mathrm{kip} i \mathrm{n} \\
& s=19.14 \mathrm{in}^{3} \\
& \sigma=\frac{m c}{I}=\frac{314,960}{19.14}=16.455 \mathrm{hsi}
\end{aligned}
$$

Shear stress

Plate shear

$$
\uparrow=U / A=\frac{93.322}{9.375(3.5)}=284 \cdot 1 \text { psi }
$$

punching Shear

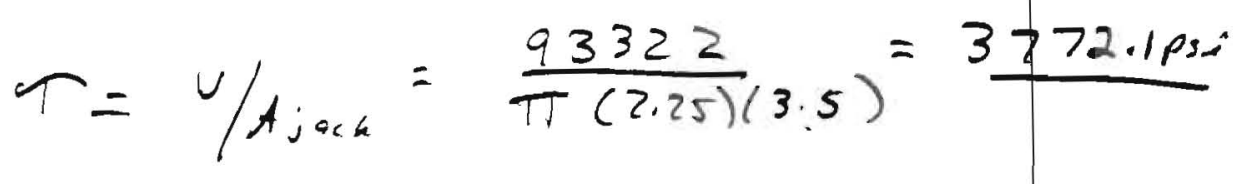

Bearing Stress

$$
F=P / A=\frac{93322 \times 10^{3}(4)}{\pi(2.25)^{2}}=\frac{23.47 \mathrm{ksi}}{1}
$$


Number one stress

Tie Plate Figure 14

$$
\begin{aligned}
& \sum m_{4}=0 \\
& R / 2(9.5)-P / 4(56.2)+F(17.25)=0 \\
& \quad F=529260.7416 \mathrm{~s} \\
& \sigma=P / A=\frac{529260.74}{(69-22)(1.5)}=7507 p \mathrm{si}
\end{aligned}
$$


Number Three Stress Calculations

Center Beam

Using Figure 9 ard the $N \omega-5 \omega$ configuration from table 1 , a free body diagram with shear and moment diagrams can be drawn.

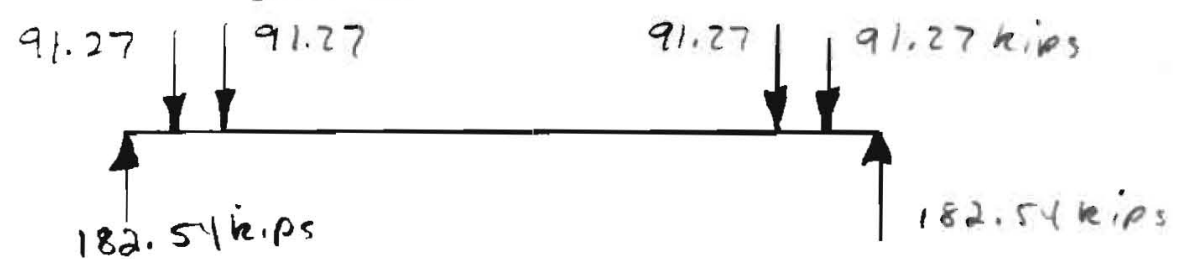

$\checkmark$

$228 \cdot 1.75 \cdot-$

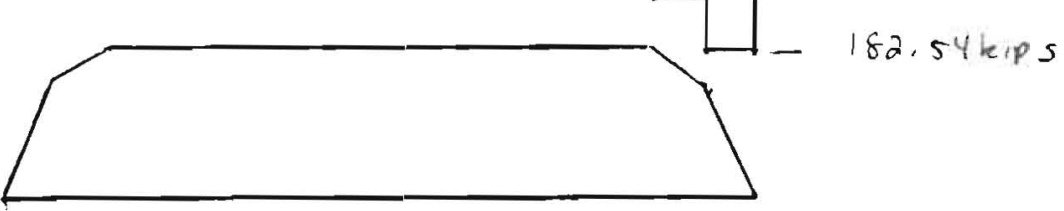

$m_{\max }=2281.75$ kip in $\quad V_{\max }=182.54 \mathrm{kips}$

Bending

Section B-B - Figure 11

$$
\begin{aligned}
I=4654 \mathrm{in}^{4} ; C & =11.179 \mathrm{in} \\
\sigma=\frac{m c}{I} & =\frac{2281.75 \times 10^{3}(11.179)}{4654}=5470.8951
\end{aligned}
$$

Section A-A Figure 10

$$
\begin{aligned}
& m=182540\left(5.5^{\prime \prime}\right)=100397016 \text {-in } \\
& c=8.867 j=1157 \\
& \sigma=\frac{m c}{I}=\frac{1003970(8.867)}{1157}=7694.21 \mathrm{psi}
\end{aligned}
$$

Shear Stress - Figure 12

Shear between Webs - Section B.B

$$
V=91270 \mathrm{lbs}
$$

$$
\psi=U / A=\frac{91270}{2(1.5)(16.5)}=1843.84 p 5 \hat{i}
$$


Number Three | Stress

Shear at $A-A$

$$
P=182,540 \mathrm{lbs} \quad T=P / A=\frac{182540}{2(1.5)(8.75)}=6953.9 \rho 5 \mathrm{i}
$$

Arms - Figure B.

Since there is no change of the load on the arms, the stresses will be exactly the same as the static Case.

Stanchion Plate- Figure 15

Since there is no change of load on stanchion plate, there will be no change in stresses

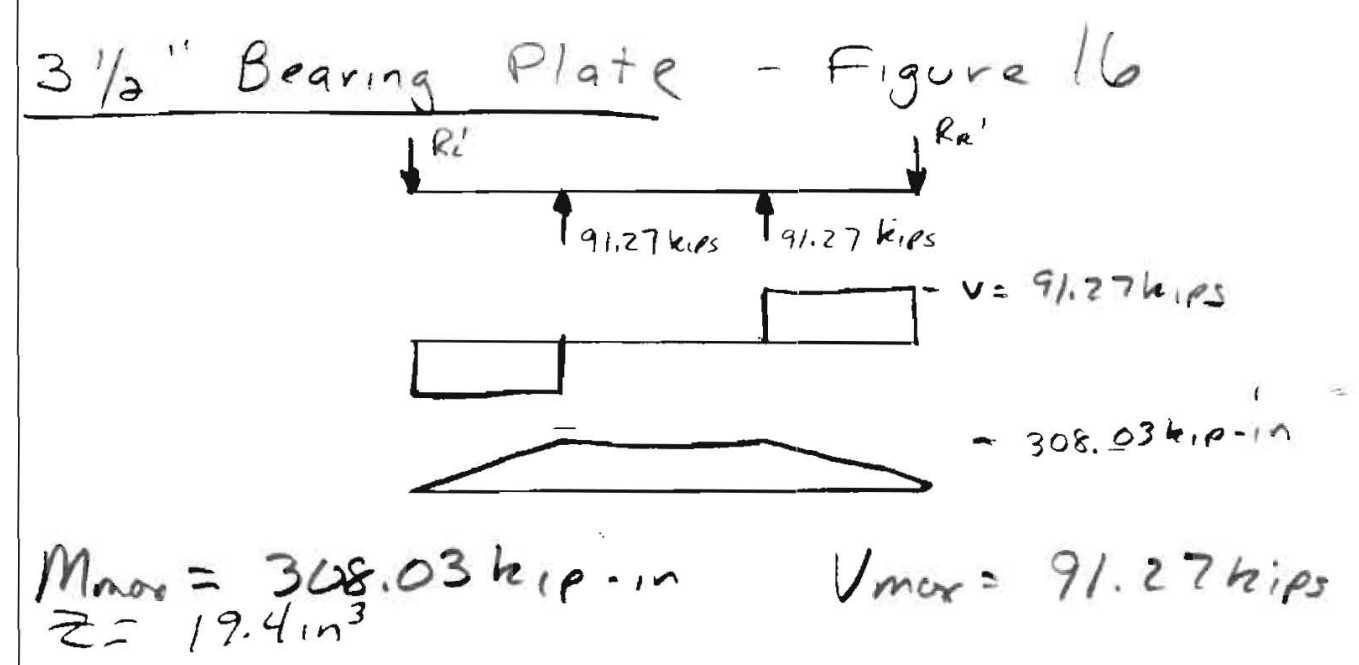

Bending Stress

$$
\sigma=\frac{M c}{I}=\frac{m}{Z}=\frac{308030}{19.4}=15877.8 \mathrm{ps}
$$

Shear Stress

Plate Shear

$$
\tau=v / A=\frac{91270}{9.375(3.5)}=2172 p 5 i
$$

Punching Shear

$$
T=U / A=\frac{91270}{\pi(2.25)(3.5)}=3689.1699 \hat{i}
$$

Bearing Stress

$$
F=P / A_{\text {ger }}=\frac{91.270(4)}{\pi(2.25)^{2}}=22,954.8 \mathrm{psi}
$$


Numberthree Stress

Tie Plate - Figure 14

$$
\begin{array}{rl}
\sum m_{A}=0 \quad R & =182510 \mathrm{lbs} P=7 / 2905 / \mathrm{bs} \\
R / 2(9.5)-P / 4(56.2)+F(17.25)=0 & F=530391.32 \\
\sigma=F / A & =\frac{530391.32}{(69.22)(1.5)}=7523.28 \mathrm{Psi}
\end{array}
$$


Number Four Stress Calculations

Center Beam

Using Figure 9 and the NE -SE configuration of table 1., Gree body, shear and moment diagram

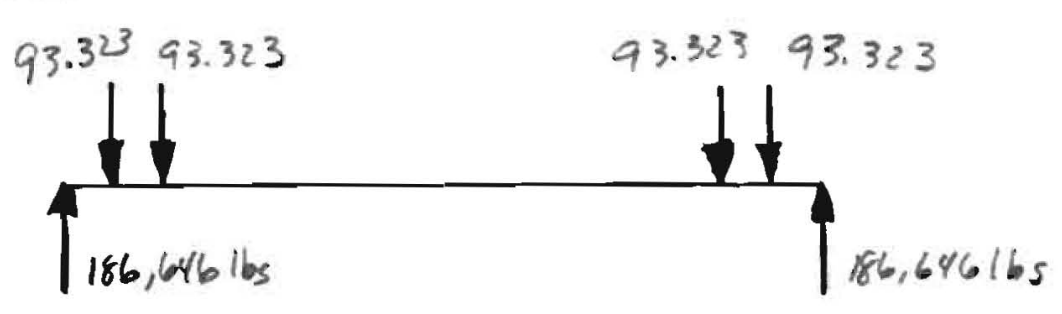

186,616

$\checkmark$

$2333.075^{2}$

$m$

$M_{\text {max }}=2333.075 \mathrm{kips}$ - in $V_{\text {mar }}=186.646 \mathrm{kips}$

Bending

Section $B-B$ - Figure 11

$$
\begin{aligned}
& I=4654 \mathrm{in}^{3} ; C=11.179 \mathrm{~m} \\
& \sigma=\frac{m c}{I}=\frac{2333.075 \times 10^{3}(11.179)}{4654} \\
& =-560 \% .09 p 5 i \\
& m=186.616 \times 0^{3}(5.5)=102655316-\mathrm{in} \\
& I=1157 \mathrm{n}^{4} ; C=8.867 \\
& \sigma=\frac{m c}{I}=\frac{1026553(8.867)}{1157}=7867.28 \mathrm{psi}
\end{aligned}
$$

Shear Stress - Figure 12

shear between wells - Section B-B

$$
V=93,323 \mathrm{lbs} T=V / A=\frac{93323}{2(1.5)(16.5)}=1885.3 \mathrm{psi}
$$


Number four Stress

Shear at $A-A$

$P=186646 \mathrm{lbs}$ of shear

$$
\sigma=P / A=\frac{1866 \% 6}{2(1.5)(8.75)}=7110.324 \rho s i
$$

Arm: Stanchion plate -Figure $13: 15$

Since there is no change in the loads on the Cradle the stresses in the Arms and Stanchion plate does not change

$3 / 2 "$ Bearing Plate - Figure 16

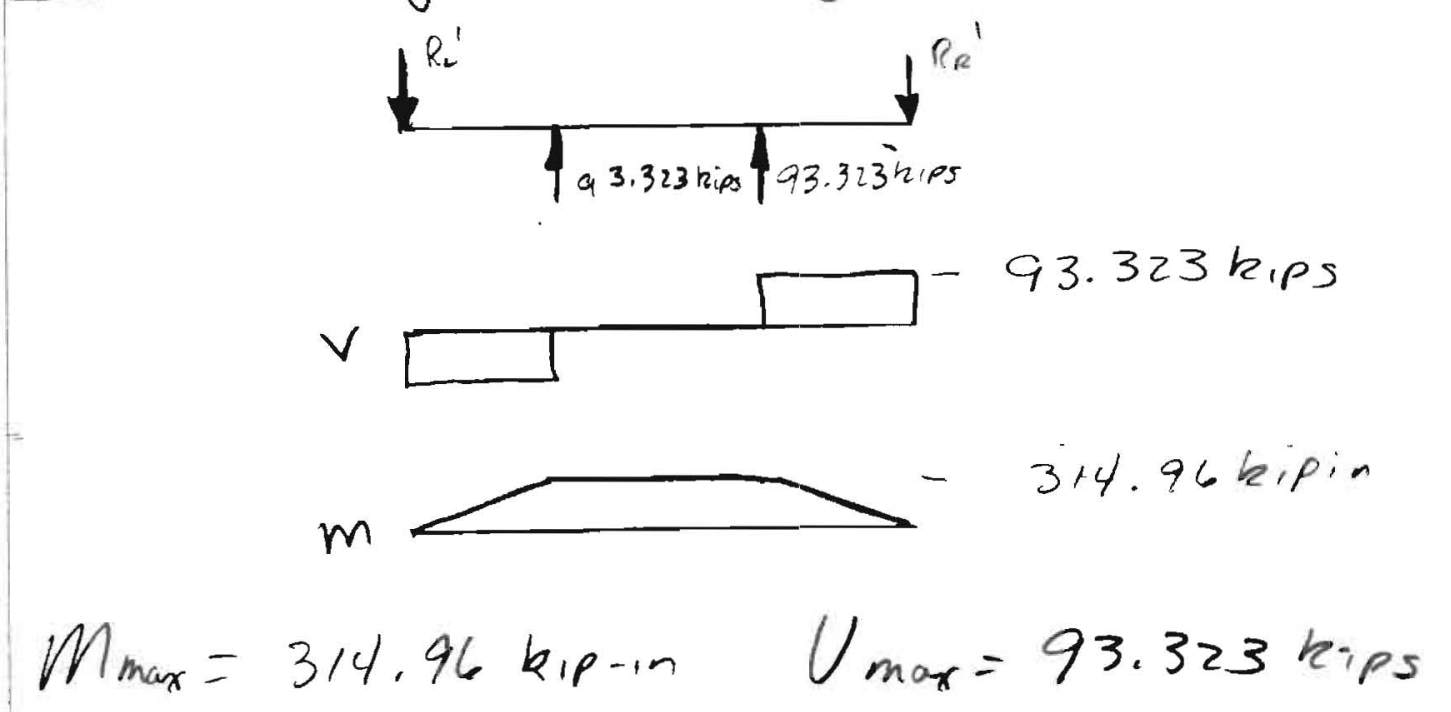

Bending

$$
\begin{aligned}
& z=19.14 \mathrm{in}^{3} \\
& \sigma=\frac{m}{z}=\frac{314.96 \times 10^{3}}{19.14}=16,455.6 \mathrm{psi}
\end{aligned}
$$

Shear stress

Plate Shear

$$
\begin{aligned}
& V=93,323 \mathrm{lbs} \\
& \text { Punching shear } T=V / A=\frac{93,323}{9.375(3.5)}=2844.13 \mathrm{psi} \\
& T=V / A=\frac{93,323}{\pi(2.25)(3.5)}=3772.14 \mathrm{psi}
\end{aligned}
$$

Bearing Stress

$$
F=93,323 \mathrm{hs} \quad F_{b}=F / A=\frac{93,323}{\pi(2.75)^{2}}=23.471 \mathrm{ksi}
$$




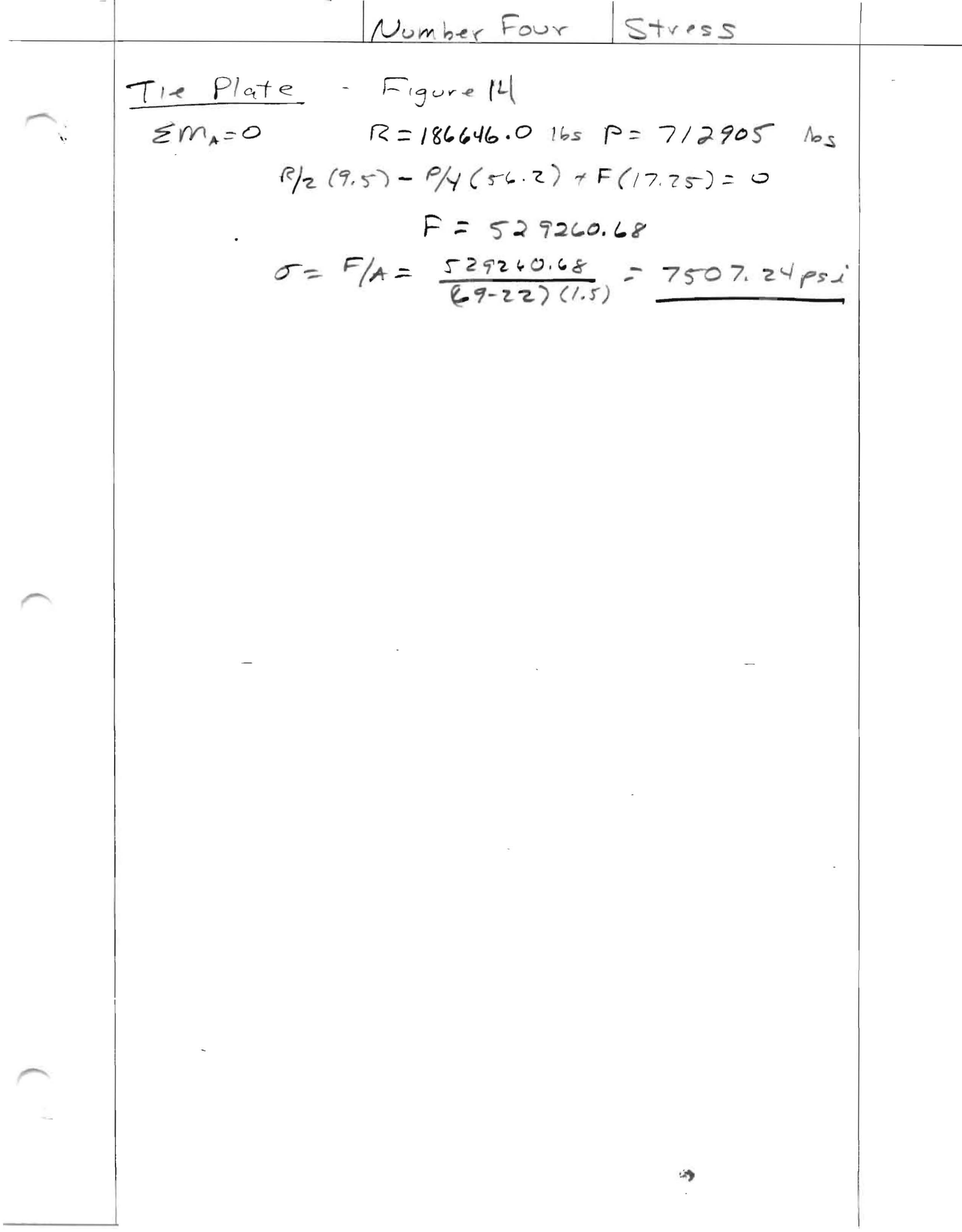


Number Five Stress Calculations

Center Beam

Using, Figure 9 ard the NE-SE configuration of table 1, a free body, shear, ard moment diagram can be drawn.

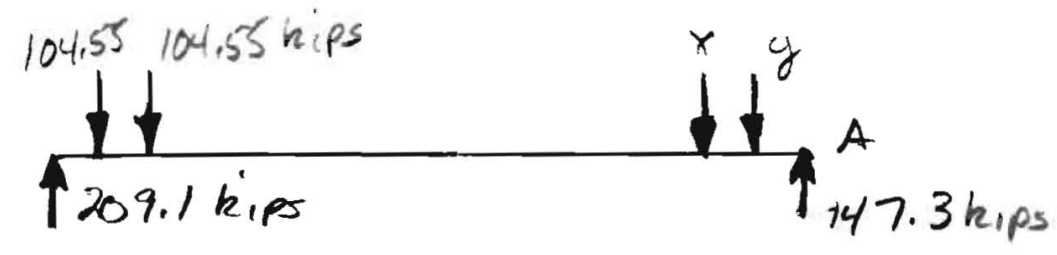

$$
\begin{array}{cc}
\varepsilon M_{A}=0 & 209.1 h_{1}(80)-104.55(73.125)-104.55(61.875) \\
& -x(18.125)-y(6.875)=0 \\
\varepsilon F=0 \quad x+y=147.3 \text { kips }
\end{array}
$$

solving for $x$ i. $y$

$$
\begin{aligned}
& x=142.3 \text { kips } \\
& y=4.983 \text { kips }
\end{aligned}
$$

209.1
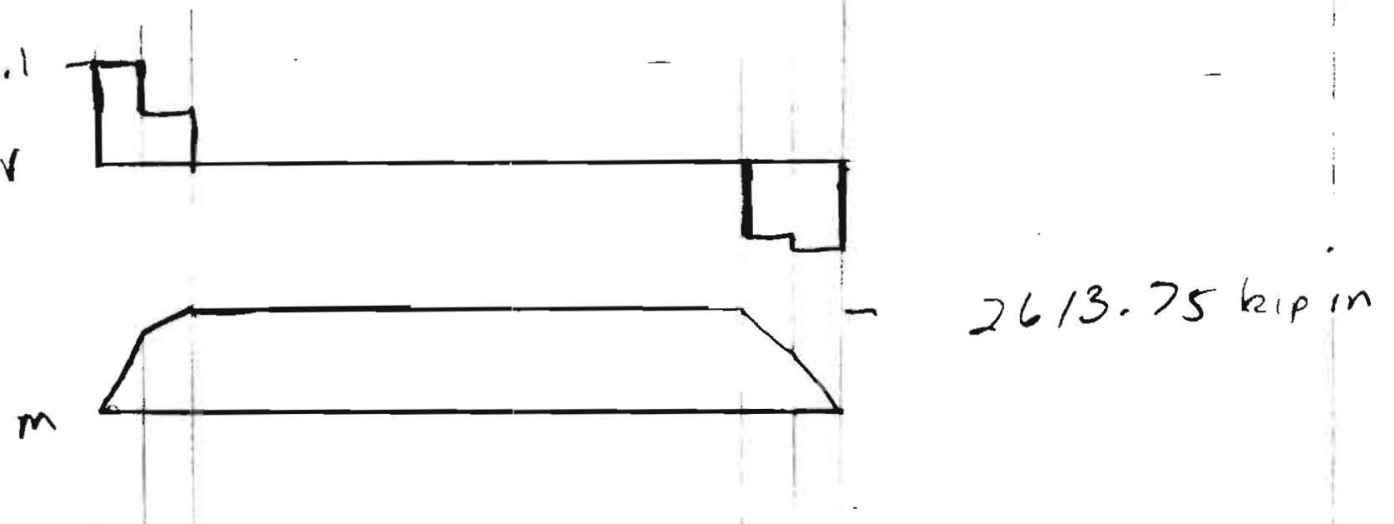

Bending

Section B-B - Figure ll

$$
\begin{aligned}
& I=4654 \mathrm{in}^{4} ; C=11.179 \mathrm{in} \\
& \sigma=\frac{m c}{I}=\frac{2613750(11.179)}{4654}=5604.09 p 5 i
\end{aligned}
$$

Section $A-A$ - Figure 10 - I= $1157 \mathrm{in} ; 6.8 .867 \mathrm{in}$

$$
\begin{aligned}
& m=209100(5.5)=1150050 \mathrm{lbs} \text { in } \\
& \sigma=\frac{m c}{I}=\frac{1150050(8.867)}{1157}=8813.7 p s i
\end{aligned}
$$


Numberfive

Stress

Shear Stress. Figure 12

Shear between webs - Section B-B

$$
V=104550 \mathrm{ks}, T=V / A=\frac{104550}{2(1.5)(16.5)}=2112.12 p_{\mathrm{si}}
$$

Shear at section A-A

$$
v=209100 \quad T=U / A=\frac{209100}{2(1.5)(8.75)}=7965.7 p 5 i
$$

Arms i Stanchion Plate - Figures $13: 15$

Since there is no change in the loads on the Arms or Stanchion Plates, the stresses in these will not change

$3 \% "$ Bearing Plate - Figure 16
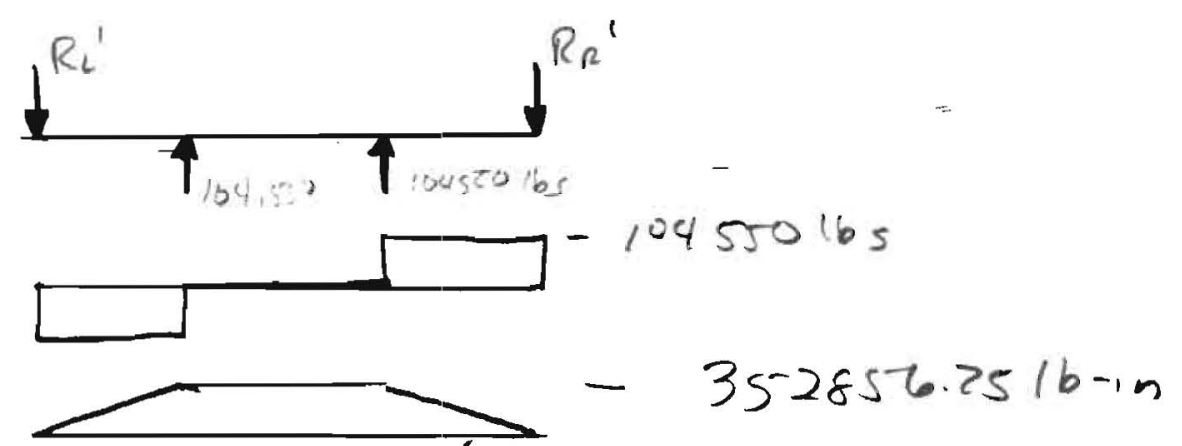

$m_{\text {max }}=352856.25 \mathrm{lb}$ in $V_{\text {max }}=1045501 \mathrm{hs}$

Bending

$$
\begin{aligned}
& z=19.4 \\
& \sigma=m / z=\frac{352856.25}{19.4}=18,188.46 \mathrm{ps}^{2}
\end{aligned}
$$

Shear

Plate Shear

$$
v=104550 \quad \Psi=v / A=\frac{104550}{9.325(3.5)}=3186.286 \text { psi }
$$


